SYSTEMS OPTIMIZATION LABORATORY

DEPARTMENT OF OPERATION RESEARCH

STANFORD UNIVERSITY

STANFORD, CALIFORNIA 94305-4022

PLANNING UNDER UNCERTAINTY

Solving Large-Scale Stochastic Linear Programs

by

Gerd Infanger

TECHNICAL REPORT SOL-92-8

December 1992

Research and reproduction of this report were partially supported by the National Science Foundation Grant ECS-8906260; U.S. Department of Energy Grant DE-FG03-92ER25116; Office of Naval Research Contract N00014-89-J-1659, and Electric Power Research Institute Contract RP8080-09; and the Austrian Science Foundation, "Fonds zur Förderung der wissenschaftlichen Forschung," Grant J0323-PHY.

Any opinions, findings, and conclusions or recommendations expressed in this publication are those of the author and do NOT necessarily reflect the views of the above sponsors.

Reproduction in whole or in part is permitted for any purposes of the United States Government. This document has been approved for public release and sale; its distribution is unlimited. 


\section{PLANNING UNDER UNCERTAINTY}

Solving Large-Scale Stochastic Linear Programs

GERD INFANGER 
Author:

Dr. Gerd Infanger

Department of Operations Research

Stanford University

Stanford, California 94305-4022, USA

Institut für Energiewirtschaft

Technische Universität Wien

Gußhausstraße 27-29

A-1040 Wien, Austria

Copyright (C) December 1992 by Dr. Gerd Infanger 
To my parents

Josef and Maria Infanger 


\begin{abstract}
For many practical problems, solutions obtained from deterministic models are unsatisfactory because they fail to hedge against certain contingencies that may occur in the future. Stochastic models address this shortcoming, but up to recently seemed to be intractable due to their size. Recent advances both in solution algorithms and in computer technology now allow us to solve important and general classes of practical stochastic problems. We show how large-scale stochastic linear programs can be efficiently solved by combining classical decomposition and Monte Carlo (importance) sampling techniques. We discuss the methodology for solving two-stage stochastic linear programs with recourse, present numerical results of large problems with numerous stochastic parameters, show how to efficiently implement the methodology on a parallel multi-computer and derive the theory for solving a general class of multi-stage problems with dependency of the stochastic parameters within a stage and between different stages.
\end{abstract}




\section{ACKNOWLEDGEMENTS}

Planning under uncertaijity and solving stochastic problems by combining decomposition techniques, Monte Carlo sampling techniques and parallel processors is a theme composed by Professor George B. Dantzig, while the use of importance sampling instead of crude Monte Carlo sampling has been recommended by Professor Peter W. Glynn. At the beginning of 1989 the author joined the Department of Operations Research at Stanford University as a visiting scholar from Vienna University of Technology, and started to work closely with Professor Dantzig on the development of techniques for solving large-scale stochastic linear programs. Progress on this topic has led to extensions of the author's visit and to his current position of Senior Research Associate at the Department of Operations Research at Stanford University.

It has been an outstanding and influential experience for the author to know and to collaborate with Professor George Dantzig. The author wants to thank Professor Dantzig for everything, all his advice, his outstanding professional support and his great friendship.

The author wants to thank Professor Peter Glynn for the many valuable discussions on the topic of planning under uncertainty and importance sampling and his great support.

The author is grateful to Professor Michael Saunders and Dr. John Stone for the valuable discussions on the topic of large-scale systems and their important and helpful suggestions concerning previous versions of this paper.

The author collaborated with Professor James K. Ho on using parallel processors. The author wishes to thank Professor Ho for this collaboration.

Thanks also to David Morton for valuable comments on a previous version of this paper and to Alamuru Krishna, who assisted in preparing some of the test problems.

The author is especially grateful to Professor Peter Jansen, his "Doktorvater", for initiating the author's visit to Stanford University and his continuing great support. 


\section{Contents}

Acknowledgements $\quad$ v

Summary 1

1 Introduction 3

1.1 Dynamic Systems under Uncertainty . . . . . . . . . . . 3

1.2 The Stochastic Optimization Problem . . . . . . . . . . . 5

1.2.1 The "Wait and See" Approach . . . . . . . . . . . . 6

1.2.2 The "Here and Now" Approach . . . . . . . . . . . 6

1.2.3 The Expected Value Approach . . . . . . . . . . . . . . 7

1.2.4 Assessment of the Different Approaches . . . . . . . . . . 7

1.2.5 The Classical Stochastic Linear Program with Recourse . . . . 8

1.2.6 Research in Stochastic Optimization . . . . . . . . . . 9

2 Benders Decomposition and Importance Sampling 13

2.1 Two-Stage Stochastic Linear Programs . . . . . . . . . . . . . . 13

2.2 Benders Decomposition . . . . . . . . . . . . . . . . . . . 14

2.3 Monte Carlo Sampling . . . . . . . . . . . . . . . . . . . 18

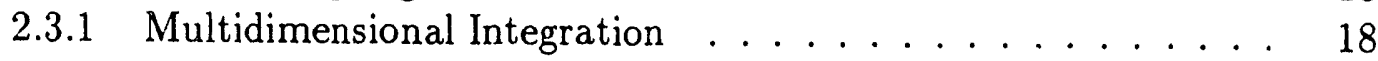

2.3.2 Crude Monte Carlo . . . . . . . . . . . . . . . . . . . 19

2.3.3 Importance Sampling . . . . . . . . . . . . . . . . . . 20

2.4 Probabilistic Cuts . . . . . . . . . . . . . . . . 24

2.4.1 The Influence of the Estimation Error . . . . . . . . . . 24

2.4.2 The Estimation Error of a Cut as a Function of $x \ldots \ldots$. . . 26

2.4.3 Upper and Lower Bounds . . . . . . . . . . . . . . . . 27

2.4 .4 Stopping Rule . . . . . . . . . . . . . . . . . 30

2.4 .5 Confidence Interval . . . . . . . . . . . . . . . . . 30

2.4 .6 Improvement of the Solution . . . . . . . . . . . . . . . 31

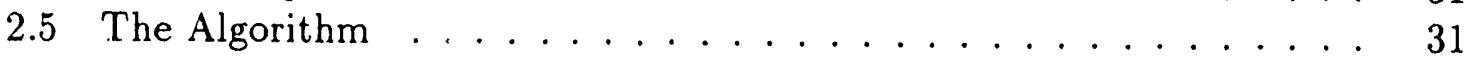

2.6 A Class of Multi-Stage Stochastic Linear Programs . . . . . . . . 32

2.7 Numerical Results . . . . . . . . . . . . . . . . . . . . 35

2.7.1 Illustrative Example . . . . . . . . . . . . . . . . . 35

2.7.2 Test Problems from the Literature . . . . . . . . . 38 
2.7.3 Large-Scale Test Problems . . . . . . . . . . . . . . . 39

3 Using Parallel Processors $\quad 45$

3.1 Hypercube Multicomputers . . . . . . . . . . . . . 45

3.2 The Parallel Algorithm . . . . . . . . . . . . . . . . 47

3.3 Performance Measures . . . . . . . . . . . . . . . . . 51

3.4 Numerical Results for the Parallel Implementation . . . . . . . . . . 53

4 Techniques for Solving Multi-Stage Problems $\quad 59$

4.1 Multi-Stage Stochastic Linear Programs . . . . . . . . . . . . 59

4.2 Dependency Among Stochastic Parameters . . . . . . . . . . . . 62

4.2 .1 Intra-Stage Dependency . . . . . . . . . . . . . . 62

4.2 .2 Inter-Stage Dependency . . . . . . . . . . . . . 63

4.3 Dual Decomposition . . . . . . . . . . . . . . . . 64

4.4 Properties of the Cuts . . . . . . . . . . . . . . . 67

4.4.1 The Case of Independence of Stochastic Parameters Between

Stages . . . . . . . . . . . . . . . . 67

4.4.2 The Case of Dependency of Stochastic Parameters Between Stages 74

4.4 .3 Summary . . . . . . . . . . . . . . . 75

4.5 Probabilistic Lower Bounds . . . . . . . . . . . . . 76

4.5.1 Estimates of Expected Values . . . . . . . . . . . 76

4.5.2 The Lower Bound Estimate . . . . . . . . . . . . . . . . 77

4.5.3 The Upper Bound Estimate . . . . . . . . . . . . . . 82

4.6 The Algorithm . . . . . . . . . . . . . . . . . . . . . 82

4.7 Computational Experience . . . . . . . . . . . . . . . 85

4.8 Applications of Multi-Stage Stochastic Linear Programs . . . . . . . 86

4.8.1 The Control of Hydro Power Systems . . . . . . . . . 86

4.8.2 The Multi-Period Asset Allocation Problem . . . . . . . 89

$\begin{array}{ll}\text { Conclusion } & 93\end{array}$ 


\section{SUMMARY}

An important class of problems of optimal allocation of scarce resources over time can be formulated as dynamic linear systems. While efficient techniques have been developed to solve large deterministic dynamic linear systems, the solutions obtained frcm these systems turned out to be unsatisfactory, because they failed to hedge against certain contingencies that may occur in the future. Stochastic models address these shortcomings but have not been widely used so far because they seemed to be intractable because of their size.

A novel approach composed by G.B. Dantzig and P. Glynn and developed jointly by them and the author combines classical decomposition techniques with a relatively new technique called importance sampling. Our approach has turned out to be capable of solving large-scale stochastic linear programs with numerous stochastic parameters which hitherto have not been able to be solved.

The basic concept for solving two-stage stochastic linear programs is as follows. Using dual (Benders) decomposition we decompose the stochastic linear program into a master problem (the first-stage problem) and into a series of subproblems (the second-stage problems), where each subproblem corresponds to a scenario, that is a certain combination of outcomes of the uncertain parameters in the model. We iteratively solve the master problem to obtain a first-stage trial solution for the problem and a series of subproblems to evaluate that trial solution, that is, to obtain the expected second-stage costs and its sensitivity with respect to the trial solution. The second stage information is passed to the master problem in form of Benders cuts; an updated trial solution is obtained in each Benders iteration. The algorithm terminates when a particular trial solution can be declared optimal. Instead of solving all possible scenarios subproblems to compute the expected future costs exactly (which is impossible even for a small number of uncertain parameters), we use Monte Carlo importance sampling techniques to obtain estimates of the expected future costs and their sensitivity. Importance sampling is crucial in our concept. It is a powerful variance reduction technique used to obtain accurate estimates with only a small sample size. Thus, the number of subproblems that has to be solved in each Benders iteration can be kept small.

We first developed the theory for solving general two-stage stochastic linear programs with recourse and a certain restricted class of multi-stage problems, for which the problem breaks down into two parts, a deterministic dynamic part and a stochastic part. The latter structure arises from the formulation of facility expansion planning problems. Our implementation uses MINOS as a subroutine for solving linear subproblems. Numerical results from large-scale test problems in the areas of facility expansion planning and financial planning demonstrated that very accurate solutions of stochastic linear programs can be obtained with only a small sample size.

The large-scale test problems included various stochastic parameters. For example, the largest problem representing expansion planning for multi-area electric power systems included 39 stochastic parameters. In the deterministic equivalent formula- 
tion, if it were possible to state it, the problem would appear as a linear program with about 4.5 billion constraints and variables. The largest portfolio optimization problem included 52 stochastic parameters, which in the deterministic equivalent formulation would appear as a linear program with about $10^{27}$ constraints and a similar number of variables. Problems of these size hitherto seemed to be intractable. Using our method we have been able to solve them on a laptop 80386 computer.

The test results indicate that we have not yet reached the limits of the approach. The sample sizes turned out to be so small that use of parallel processors is not a condition sine qua non for solving even large-scale stochastic linear problems. In order to speed-up the computation time in the case where large sample sizes are required, we have developed a parallel implementation running on a hypercube multi-computer. The numerical results show that speed-ups of about $60 \%$ can be obtained using 64 parallel processors.

Encouraged by the promising numerical results for two-stage and a restricted class of multi-stage problems we then have developed the theory for a general class of multistage stochastic linear programs. Our approach for solving multi-stage problems, includes special sampling techniques for computing upper bounds and methods of sharing cuts between different sub-problems. It will enable us to efficiently solve largescale multi-stage problems with many stages and numerous stochastic parameters in each stage. The implementation is subject to future research. Preliminary numerical results have turned out to be promising.

Further research includes improved decomposition techniques for large-scale problems, e.g., optimized tree traversing strategies and passing information based on nonoptimal subproblems, improvements to the importance sampling approach, e.g., using different types of approximation functions, improved software, e.g., a parallel implementation of the multi-stage algorithm on distributed workstations, and the testing of the methodology on different practical problems in different areas. 


\section{Chapter 1}

\section{Introduction}

\subsection{DYNAMIC SYSTEMS UNDER UNCERTAINTY}

A fundamental economic problem is the optimal allocation of scarce resources over time. Since Dantzig (1948) [20] inven'ed the simplex method for linear progran.ming (see Dantzig (1963) [22]), Operations Research has been developing efficient techniques to address this important problem. Important developments of Operations Research include recent advances in linear, nonlinear, and discrete optimization techniques, and especially the advancements of large-scale optimization techniques. For basic references see e.g. Gill, Murray, Wright (1981) [55], (1991) [56], Lasdon (1970) [85] and Geoffrion (1974) [57]. Recent advances in computer technology, e.g. vector processing and distributed computation on parallel processors, contribute to the capabilities of modern Operations Research methods.

An important class of resource allocation problems over time can be formulated as dynamic linear systems. These are linear programs with a certain matrix structure. The non-zero elements of the constraint matrix appear in a staircase pattern, where each step of the staircase corresponds to a certain time period. This structure emerges because constraints associated with a particular time period have coefficients in that time period and in the period before, for all time periods of the planning horizon. Systems of this kind are also called multi-stage linear programs. Note that optimal linear control problems in discrete time also fall into this category.

Multi-stage linear programs have been studied extensively. Methods for solving staircase systems exploit their structure in order to increase the computational efficiency. Dantzig and Wolfe's (1960) [31] primal decomposition and nested dual decomposition based on Benders (1962) [6] have been exploited and further developed. Ho and Loute (1981) [69] gave efficient implementations and collected a set of staircase linear programming test problems. Some references for techniques for solving dynamic systems are Glassey (1973) [65], Ho and Manne (1974) [70], Abrahamson (1983) [1] and Wittrock (1983) [130]. Parallel dual decomposition has been applied by Entriken (1988) [39], (1989) [40], and parallel primal decomposition by Ho and Gnanendran (1989) [67] and Ho, Lee and Sundarraj (1988)[68].

While efficient techniques allow us to solve dynamic systems of very large sizes, e.g. problems can be solved with several hundred thousand variables, the solutions obtained from these systems have often proved to be impractical. Parameters of the system, which had been assumed to have certain values when the model was formu- 
lated, assumed different values when the optimal solution was finally implemented. These were deterministic systems, where all parameters were assumed to be known to the planner with certainty. Solutions obtained from deterministic planning models fail to hedge against different contingencies that occur unpredicably in the future.

Consider, for example, an operations planning problem of an electric power system. An operations plan is determined, subject to constraints based on certain availabilities of generators and transmission lines, and certain values of demands. The optimal solution obtained is only optimal for this particular choice of parameters. When the optimal solution is implemented, the generators and transmission lines may assume different values of availability, e.g. due to unplanned failure, and the demands may become different from the planned values. For these values of the parameters of the system the solution is no longer optimal. Difierent costs than planned are the consequence. In extreme situations the implemented solution may lead to infeasibility, when unfavorable values occur. For example, the demands may not be able to be satisfied, and very costly actions may be necessary.

As a further example consider the portfolio optimization problen. A cieterninistic approach would assume the returns of the equities traded at the market as kiown parameters. The optimal solution of the deterministic model would contain fractic ns of equities in decreasing order of their planned returns, subject to the constraints and bounds in the model. It would contain as many shares as possible of the equity with the highest assumed return, then as many as possible with the second highest return, and so forth. An investor having implemented this optimal solution (that is having bought the recommended numbers of shares at the market) may be disappointed when he later observes different values of returns than have been assumed in the model. The returns of a portfolio, selected by a deterministic model, may be significantly different than expected. Of course, no one would attempt to implement the solution of a deterministic portfolio optimization problem.

Since solutions obtained from deterministic planning models have turned out to be unsatisfactory, different techniques have been developed to compensate for their shortcomings.

- Sensitivity analysis examines the changes of the optimal solution and the optimal objective value with respect to variations of uncertain parameters that are considered to be important. Usually sensitivity analysis is conducted by varying one parameter at a time. Linear programming theory provides for local sensitivity results as a post-optimal analysis; see, e.g., Dantzig (1963) [22]. Usually not only local sensitivity analysis is performed, but uncertain parameters are varied according to their full range of possible outcomes. If the solution of the problem turns out to be very sensitive to a particular parameter, the value of this parameter is revised to be more on the safe side. Then, by solving the optimization problem again, one hopes to find a new solution that accounts, at least partially, for the uncertainty. Clearly, sensitivity analysis cannot overcome the shortcomings of deterministic planning models. 
- Scenario analysis is another method that has been widely us'ed and seems to be the preferred technique for many planners. In this approach one assumes scenarios (certain combinations of possible values of the uncertain parameters) and solves the problem for each of these scenarios. The different optimal solutions and the corresponding optimal objective values in the different scenarios are then aggregated in a heuristic way. By conducting scenario analysis the planner hopes to get insight into the problem. By solving the problem repeatedly for different scenarios and studying the solutions obtained the planner observes sensitivities and heuristically decides on an appropriate solution.

- Worst case analysis and other related techniques attempt to account for uncertainty by putting safety margins into the problem formulation.

On the other hand, stochastic models address the shortcomings of deterministic models directly. Instead of assuming the uncertain parameters of the model to be known, stochastic models assume their distributions to be known. This causes the model size to grow enourmously, as will be discussed in detail later. As a result, it has been too difficult to solve real world practical stochastic models and they have not been widely used so far. A new approach, based on decomposition techniques and Monte Carlo importance sampling, has been composed by Dantzig and Glynn (1990) [24] and developed by them and Infanger (1991) [73]. We believe that this approach is a breakthrough in solving problems of planning under uncertainty. In the following we will discuss this approach.

We first outline the basic theory for two-stage stochastic linear problems and extend the discussion to a special class of multi-stage problems. We demonstrate the power of the method on numerical results obtained from different test problems. We then discuss an implementation of the method using parallel processors which we have developed in collaboration with Ho (Dantzig, Ho, Infanger (1991) [26]). Finally we derive the theory of solving general multi-stage stochastic linear problems.

First of all, we begin by introducing the stochastic problem and discussing different approaches to its solution.

\subsection{THE STOCHASTIC OPTIMIZATION PROBLEM}

Defining a probability space $(\Omega, P)$, where $\Omega$ is the set of possible realizations $\omega$ of the uncertain parameters and $P$ the corresponding probability distribution, a stochastic optimization problem can be represented as:

$$
\begin{aligned}
& z=\min _{\mathrm{s} / \mathrm{t} x \in C \subseteq(f(x, \omega))=\int f(x, \omega) d P(\omega)}\left(\omega R^{n},\right.
\end{aligned}
$$

where $f(x, \omega)$ is the objective function and $C$ the set of feasible solutions defined by the constraints of the optimization problem. The probability space $(\Omega, P)$ can represent all kinds of distributions, e.g. continuous, discrete, with a finite or infinite 
number of realizations. For example, consider the case in which $\Omega$ is discrete and finite and where a discrete outcome $\omega$ has corresponding probability $p(\omega)$. Problem (1.1) then is represented as:

$$
\begin{aligned}
& z= \min E f(x, \omega)=\sum_{\omega \in \Omega} f(x, \omega) p(\omega) \\
& \mathrm{s} / \mathrm{t} x \in C \subseteq R^{n} .
\end{aligned}
$$

Problems (1.1) and (1.2) are hard to solve. The main problem lies in the sheer number of possible outcomes that have to be taken into account. In problem (1.2) we define the number of elements in $\Omega$ to be $K=|\Omega|$. In practical problerns $K$ is a very large number. In order to fully understand the nature of a stochastic solution, we will discuss different solution approaches to the stochastic optimization problem. For simplicity and ease of exposition we conduct the discussion for whe case of $\Omega$ being discrete and finite.

\subsubsection{The "Wait and See" Approach}

In the "wait and see" approach we assume that we can somehow wait and see until the uncertainty is resolved at the end of the planning horizon and an outcome $\omega \in \Omega$ can be observed, before we make the optimal decision $x$. The "wait and see" approach therefore assumes perfect information about the future. It is clear that such a solution is not implementable. The corresponding problem can be stated as

$$
\begin{gathered}
z^{\omega}=\min _{\mathrm{s} / \mathrm{t} x \in C^{\omega} \subseteq R^{n},} f(x, \omega) \\
x^{\omega} \in \arg \min \left\{f(x, \omega) \mid x \in C^{\omega}\right\}, \\
z_{w s}=E z^{\omega}=\sum_{\omega \in \Omega} z^{\omega} p(\omega) .
\end{gathered}
$$

The set of feasible solutions of $x, C^{\omega}$, is defined by the constraints in scenario $\omega$. We solve the problem for the observed outcome $\omega$ and obtain an optimal solution subject to scenario $\omega, x^{\omega}$. It is an optimal solution given perfect information about the future. We compute the expected value of the optimal costs $z^{\omega}$ to obtain $z_{w s}$, the expected costs under perfect information.

\subsubsection{The "Here and Now" Approach}

The "here and now" approach represents the true stochastic optimization problem of (1.2). A decision $x$ has to be made "here and now", before observing an outcome of $\Omega$. The value $x$ is chosen, such that the expected costs $E f(x, \omega)$ assume a minimum:

$$
\begin{aligned}
z= & \min E f(x, \omega) \\
& \mathrm{s} / \mathrm{t} x \in C=\bigcap_{\omega \in \Omega} C^{\omega} .
\end{aligned}
$$


The optimal objective function value $z$ denotes the minimum expected costs of the stochastic optimization problem. Note that $x$ has to be feasible for all scenarios $\omega \in \Omega$; thus $C=\bigcap_{\omega \in \Omega} C^{\omega}$ denotes the intersection of all $C^{\omega}, \omega \in \Omega$, where $C^{\omega}$ represents the feasible region given by the constraints in scenario $\omega$. The optimal solution

$$
x^{*} \in \arg \min \left\{E f(x, \omega) \mid x \in \bigcap_{\omega \in \Omega} C^{\omega}\right\}
$$

represents the realistic solution of the stochastic optimization problem. The solution $x^{*}$ hedges against all possible contingencies $\omega \in \Omega$ that may occur in the future.

\subsubsection{The Expected Value Approach}

Let $\bar{\omega}$ denote the expectation over the set $\Omega$ :

$$
\bar{\omega}=E \omega=\sum_{\omega \in \Omega} \omega p(\omega) .
$$

In the expected value approach we replace the stochastic parameters by their expectated values and solve the corresponding deterministic problem:

$$
\begin{gathered}
\hat{z}_{d}=\min _{\mathrm{s} / \mathrm{t} x \in C^{\bar{\omega}}} f(x, \bar{\omega}) \\
x_{d} \in \arg \min \left\{f(x, \bar{\omega}) \mid x \in C^{\bar{\omega}}\right\}
\end{gathered}
$$

We refer to problem (1.9) as the expes:ted value problem corresponding to the stochastic optimization problem (1.2). $\hat{z}_{d}$ denotes the costs corresponding to $x_{d}$, the optimal solution of the expecied value problem. We denote by $z_{d}$ the expected costs corresponding to the implementation of the soiution of the expected value problem, $x_{d}$ :

$$
z_{d}=E f\left(x_{d}, \omega\right)
$$

\subsubsection{Assessment of the Different Approaches}

Assuming $f(x, \omega)$ to be convex and comparing the expected costs of the different solutions of the stochastic optimization problem, we can show that

$$
z_{w s} \leq z \leq z_{d}
$$

We define the expected value of perfect information, EVPI, to be

$$
\mathrm{EVPI}=z-z_{w s}
$$

It is a measure of how much one would be willing to pay (at most) to obtain perfect information about the future. A small EVPI indicates that refined forecasts will lead 
to little gain. A large EVPI indicates that incomplete information about the future is costly.

We define the value of the stochastic solution, VSS, to be

$$
\mathrm{VSS}=z_{d}-z .
$$

It is a measure of how much can be saved by implementing the solution of the stochastic optimization problem versus the solution of the deterministic expected value problem. If VSS is small, the approximation of the stochastic problem by the corresponding expected value problem is a good one and the obtained expected value solution is a good solution for the stochastic problem. The larger the value of VSS the more important it is to obtain the solution of the stochastic optimization problem.

Birge (1980) [10] develops bounds on the expected value of perfect information and on the value of the stochastic solution, e.g.:

$$
\begin{gathered}
0 \leq \mathrm{EVPI} \leq z-\hat{z}_{d} \leq z_{d}-\hat{z}_{d}, \\
0 \leq \mathrm{VSS} \leq z_{d}-\hat{z}_{d} .
\end{gathered}
$$

The bounds on EVPI and VSS are useful for deciding if it is necessary to solve the stochastic problem or if an approximation is adequate.

\subsubsection{The Classical Stochastic Linear Program with Recourse}

A classical stochastic optimization problem is the two-stage stochastic linear program with recourse. It has the form

$$
\begin{aligned}
z=\min _{\mathrm{s} / \mathrm{t}} \quad c x & +E_{\omega} Q(x, \omega)=b \\
x & \geq 0,
\end{aligned}
$$

where

$$
Q(x, \omega)=\min \begin{aligned}
f(\omega) y & \\
\mathrm{~s} / \mathrm{t} \quad D(\omega) y & =d(\omega)+B(\omega) x \\
y & \geq 0 .
\end{aligned}
$$

The matrix $A$ and the vector $b$ are known with certainty. $E_{\omega}$ denotes the expectation with respect to $\omega$, an element of the probability space $(\Omega, P)$, and the function $Q(x, \omega)$ is referred to as the recourse function, which in turn is defined by the linear program (1.18). The technology matrix $D(\omega)$, the right hand side $d(\omega)$ and the transition matrix $B(\omega)$ as well as the objective function coefficients $f(\omega)$ of this linear program are random. Since Dantzig (1955) [21], this type of problem has been studied extensively by many authors. A special class of (1.18) is that of complete recourse, where the recourse function $Q(x, \omega)$ is finite (e.g. (1.18) is feasible) for any choice of $x$. Properties of this class have been studied e.g. by Wets (1966)[124]. The recourse function $Q(x, \omega)$ can be shown to be convex and is in general non-smooth. 
To evaluate the expectation $E Q(x, \omega)$, a multiple integral or a multiple sum typically has to be computed. We will show this later. The main difficulties in stochastic optimization deal with the evaluations of multiple integrals or multiple sums. The numerical computation of expectations requires large numbers of function evaluations, where each function evaluation means that a linear program has to be solved.

There are several approaches to attack this problem. In the following we give an overview of different approaches that have been developed to solve stochastic optimiration problems.

\subsubsection{Research in Stochastic Optimization}

Stochastic programming with recourse was first introduced independently by Dantzig (1955) [21] and Beale (1955) [4]. Chance constrained stochastic programming, involving a different type of model, was first introduced by Charnes and Cooper (1959) [18]. It involves models where a decision is made prior to the knowledge of outcomes of random parameters, such that certain constraints are met with certain probability levels. See Prékopa (1988) [109] for an overview of probabilistic constrained programming models. A number of different algorithmic approaches have been proposed for solving two-stage stochastic linear programs of the type (1.17), (1.18) stated above. (See Kall (1976) [78], Wets (1974) [125] and Wets (1983) [126] for an investigation of the recourse problem.)

Van Slyke and Wets (1969) [121] showed with their L-shaped method how Benders (1962) [6] decomposition can be applied to solving two-stage stochastic linear programs. Their algorithm uses expected-value cuts, representing an outer linearization of the expected second-stage costs (or the recourse function). A variant proposed later by Birge and Louveaux (1985) [11] is based on multiple cuts, where a different cut with respect to each scenario is computed, and the expected-value calculation is carried out in the master problem. Earlier, Dantzig and Madansky (1961) [29] pointed out that the dual of the two-stage stochastic linear program has a structure ićeal for Dantzig-Wolfe (1960) [31] decomposition.

Stochastic quasigradient methods select sequentially random search directions based on a limited number of observations of the ran . sm function (1.18) in each iteration. They have been studied by Ermoliev (1983) [42], (1988) [43] [44] and Gaivoronski (1988) [51]. The convergence rates of stochastic quasigradient methods are low; it is important how well objective values, subgradients, and stepsizes can be specified. Pflug (1988) [113] provided stepsize rules and stopping criteria for stochastic quasigradient methods, and Ruszczynski (1987) [118] contributed by proposing a linearization method. Marti (1980) [92] improved the convergence by introducing semi-stochastic approximation.

A classical approximation scheme for solving two-stage stochastic linear programs with stochastic right-hand sides (randomness in the transition matrix $B$ and the right-hand side $d$, see equation (1.18)) is to calculate upper and lower bounds via 
the inequalities of Jensen (1906) [76] and Edmundson (1956) [38] and Madansky (1959) [90], respectively, and to successively improve these bounds. (See, e.g., Kall, Ruszczynski, Frauendorfer (1988) [80].) The lower bound based on Jensen's inequality involves the evaluation of the recourse function at only one point, namely the expected value of the random parameters. The upper bound due to Edmundson and Madansky is based on the theory of moment spaces and is computed by weighting the extreme points of the support of the random variables. Refinements of this bound have been proposed by Ben Tal and Hochman (1972) [7], Kall (1974) [77], Huang, Ziemba and Ben-Tal (1977) [71], Kall and Stoyan (1982) [81], Frauendorfer and Kall (1988) [50] for independent random variables, and Dupačowá (1978) [36], Gassmann and Ziemba (1986) [54], Frauendorfer (1988) [48] and Birge and Wallace (1988) for dependent random variables. Frauendorfer (1992) [49] uses Barycentric Approximations to solve two-stage stochastic programs. Ermoliev, Gaivoronski and Nedeva (1985) [45] provided a general framework for stochastic programming problems. Birge and Wets (1987) [15] and Cipra (1985) [19] computed bounds based on solving a generalized moment problem. Based on that, Birge and Wets (1986) [14] and (1989) [16] exploited the sublinear property of the recourse function. Wallace (1987) [123] proposed a procedure for the case that the evaluation of the recourse function involves the solution of a network problem, and Birge and Wallace (1988) [13] provided a separable piecewise linear upper bound. Upper bounds for the expectation of convex functions with discrete random variables and the relationship of moment problems and linear programming have been investigated by Prékopa (1988) [110], (1989) [111], and (1990) [112]. Birge (1985) [9] proposed row and column aggregation schemes to approximate a stochastic program. Robinson and Wets (1987) [114] present stability results for two-stage stochastic programs.

Using mathematical programming techniques seemed to be promising in special cases, e.g. Nazareth and Wets (1986) [100]. Strazicky (1980) [119] and Kall (1979) [79] proposed basis factorization approaches. Ruszczynski (1986) [117] proposed his regularized decomposition method. Wets (1988) [128] surveyed the use of large-scale linear programming techniques, and Nazareth and Wets (1988) [101] provided an overview of using nonlinear programming techniques for solving stochastic programs. Lustig et al. (1991) [89] empirically studied interior-point linear programming solvers' performance on different formulations of two-stage stochastic linear programs.

Rockafellar and Wets (1989) [115] presented "Progressive Hedging", in which nonanticipativity constraints are enforced via Lagrangian penalty terms in the objective, and in each iteration a two-stage or multi-stage program is solved for each scenario. It provides an iterative method, solving scenario optimization problems to the solution of the stochastic problem. Numerical results of the performance of "Progressive Hedging" have been given by Mulvey and Vladimirou (1989) [97], [98].

King and Wets (1989)[82] have applied the theory of epi-consistency to stochastic programming in order to obtain consistency results for sequences of optimal solutions, and Dupačowá and Wets (1988) [37] studied the asymptotic behavior of statistical 
estimators in stochastic programs.

Higle and Sen (1989) [61] developed a "Stochastic Decomposition" Benders Decomposition method, which, like the stochastic quasigradient algorithm, only requires one observation or a very small number of observations per iteration, and which asymtotically creates an outer linearization of the second-stage costs. Optimality conditions and stopping rules for their method have been presented in Higle and Sen (1989) [62]. Higle, Wing and Odio (1990) [64] subsequently extended the framework to the case where the recourse function is evaluated by simulation.

Gaivoronski and Nazareth (1989) [52] combined generalized programming with sampling techniques. Niederreiter (1986) [102] proposed the use of pseudo random numbers for multi-dimensional numerical integration. Deák (1988) [33] gave a survey of well known techniques for multi-dimensional integration for stochastic programming. Monte Carlo methods are known to be efficient for multi-dimensional numerical intergration. Lavenberg and Welch (1981) [86] and Rubinstein and Markus (1985) [116] discussed the efficiency of control variables in Monte Carlo simulation. Pereira et al. (1989) [104] used control variables as a variance-reduction technique in Monte Carlo sampling in a modified Benders decomposition framework. Dantzig and Glynn (1990) [24] and Infanger (1991) [73] used importance sampling, based on an additive approximation function, as a variance-reduction technique for Monte Carlo sampling for stochastic linear programs. Krishna (1992) [83] extended the scheme to using piecewise linear approximation functions.

Birge (1985) [9] extended the L-shaped method to multi-stage stochastic programs, employing a nested Benders decomposition scheme. Gassmann (1990) [53], based on results by Wittrock (1983) [130] for deterministic multi-stage programs, explored different tree-traversing strategies in a Benders decomposition framework for stochastic multi-stage programs. This work has been extended by Morton (1992) [93] and Morton and Zang (1992) [94]. Louveaux (1986) [87] discussed multi-stage problems with block-separable recourse. Beale, Dantzig and Watson (1986) [5] proposed a firstorder approach to a class of multi-stage stochastic programs. Dempster (1986) [35] also studied multi-stage problems. Pereira and Pinto (1991) [106] proposed stochastic dual dynamic programming, a dual Benders decomposition approach, exploiting the piecewise linear property of the recourse function, and introduced path sampling for obtaining estimates of upper bounds.

Ideas of using parallel processors can be found in Wets (1985) [127], Dantzig (1988) [23], Hillier and Eckstein (1990) [66], Zenios (1990) [131], and Ariyawansa and Hudson [2]. There has been a large variety of applications for stochastic programming; for example, Ferguson and Dantzig (1956) [47] allocated aircraft to routes, Kusy and Ziemba (1986) [84] formulated a bank asset and liability management model, Zenios (1992) [132] managed large mortgage-bacied securities, Mulvey (1987) [95] formulated nonlinear networks for modeling in finance, Pereira and Pinto (1989) [103] optimized large hydro-electric systems and (1991) [107] carried out energy planning. A description of practical models can be found in Dempster (1980) [34] and Ermoliev 
and Wets (1988) [46]. Overviews of stochast:: : programming are given in Dempster (1980) [34], Ermoliev and Wets (1988) [46] and Wets (1989) [129]. For a recent survey, see Birge and Wets (1991) [17]. 


\section{Chapter 2}

\section{Benders Decomposition and Importance Sampling}

\subsection{TWO-STAGE STOCHASTIC LINEAR PROGRAMS}

An important class of stochastic models is that of two-stage stochastic linear programs with recourse. These models can be seen as the stochastic extensions of deterministic dynamic systems with two stages: $x, y$ denote the first and the second-stage decision variables, $A, b$ represent the coefficients and right-hand sides of the first stage constraints, and $D, d$ concern the second period constraints together with the transition matrix $B$ that couples the two periods. In the literature, $D$ is often referred to as the technology or recourse matrix. $c, f$ are the objective function coefficients.

In the deterministic case, $c, f, A, b, B, D, d$ are known with certainty to the planner. In the stochastic case, the values of the second-stage parameters are uncertain. The second-stage parameters are known only by their probability distribution of possible outcomes at time $t=1$, but actual outcomes will be known later at time $t=2$. The second-stage parameters can be viewed as random variables that assume certain outcomes with certain probabilities. We denote a certain outcome of these random variables by $\omega$ and the corresponding probability by $p^{\omega}$ or $p(\omega), \omega \in \Omega$, the set of possible outcomes. We consider the case where uncertainty occurs only in the transition matrix $B$ and in the right-hand side vector $d$. The second-stage costs $f$ and the elements of the recourse matrix $D$ are assumed to be known with certainty.

In (2.1) a two stage staircase problem is transformed into a two stage stochastic linear program.

$$
\begin{aligned}
& \min Z=c x+E^{\omega}\left(f y^{\omega}\right) \\
& \mathrm{s} / \mathrm{t} \quad A x=b \\
& -B^{\omega} x+D y^{\omega}=d^{\omega} \\
& x, y^{\omega} \quad \geq 0, \omega \in \Omega \text {. }
\end{aligned}
$$

The problem is to find a first stage decision $x$ that is feasible for all scenarios $\omega \in \Omega$ and has the minimum expected costs. Note the adaptive nature of the problem: while the decision $x$ is made only with the knowledge of the distribution $(\omega, p(\omega))$ of the random parameters, the second-stage decision $y^{\omega}$ is made after an outcome 
$\omega$ is observed. The second-stage decision compensates for and adapts to different scenarios $\omega$.

Let us consider the case of $\Omega$ being discrete and finite, and define $\Omega$ as an index set $\Omega=\{1, \ldots, K\}$ that means the parameter $\omega$ may take on $K$ different values. Then we can formulate adeterministic problem that is equivalent to the stochastic linear problem. This deterministic equivalent problem is tractable only if $K$ is small. It takes the form

$$
\begin{aligned}
& \min Z=\quad c x+p^{1} f y^{1}+p^{2} f y^{2}+\cdots+p^{K} f y^{K} \\
& \mathrm{~s} / \mathrm{t} \quad A x \quad=b \\
& -B^{1} x+D y^{1}=d^{1} \\
& -B^{2} x+D y^{2} \\
& \begin{array}{rlll}
\vdots & & \ddots & \\
-B^{K} x & & & \\
x, & y^{1}, & y^{2}, \quad \ldots, \quad D y^{K} & =d^{K} \\
y^{K} & \geq 0 .
\end{array}
\end{aligned}
$$

In the deterministic equivalent problem (2.2) the second-stage constraints are explicitly formulated for each scenario $\omega \in \Omega$, one below the other. The objective function carries out the expected value computation by direct summation. Clearly, this formulation may lead to linear programs of enormous size.

\subsection{BENDERS DECOMPOSITION}

The method we wish to apply to solve two-stage stochastic linear programs utilizes Benders (1962) [6] decomposition. Van Slyke and Wets (1969) [122] suggested expressing the expected value of the second-stage costs by a scalar $\theta$ and replacing the second-stage constraints sequentially by "cuts", which are necessary conditions expressed only in terms of the first stage variables $x$ and $\theta$. Our analysis follows this approach.

In the following we will derive the main steps of a Benders decomposition algorithm for two-stage stochastic linear programs. We will consider the "universe" case, which yields the exact solution of the equivalent deterministic problem ("certainty equivalent"). See, e.g., Geoffrion (1974) [57] for an excellent derivation of the Benders decomposition algorithm.

Given the equivalent deterministic problem in (2.2) and assuming $K$ scenarios describe the universe case, we rewrite the problem applying projection onto the $x$ variables and obtain (2.3). We assume for simplicity that (2.2) is feasible and has a 
finite optimum solution.

$$
\begin{aligned}
& \min Z=
\end{aligned}
$$

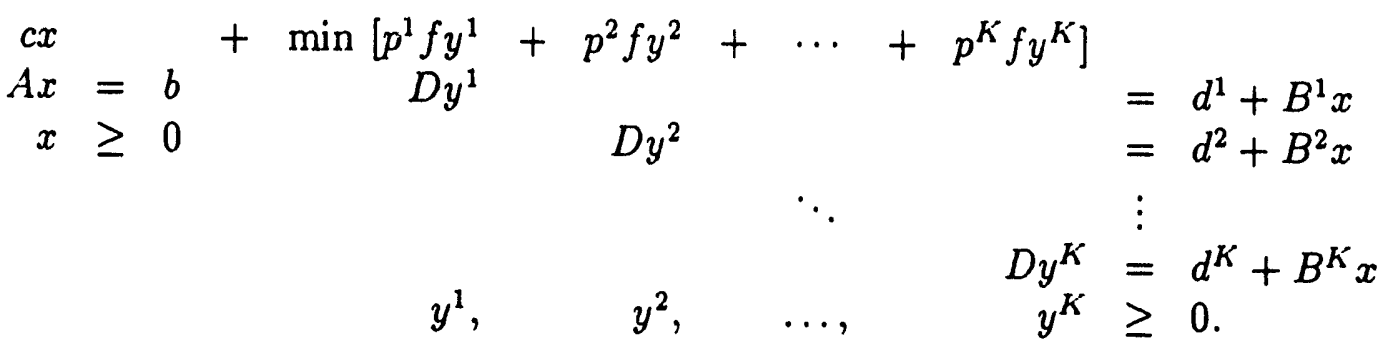

The infimal value function in (2.3) corresponds to the following primal linear problem (2.4):

$$
\begin{aligned}
& \min z_{P}=p^{1} f y^{1}+p^{2} f y^{2}+\cdots+p^{K} f y^{K}=E^{\omega}\left(f y^{\omega}\right) \\
& p^{1} \pi^{1}: \quad D y^{1}=d^{1}+B^{1} x \\
& p^{2} \pi^{2}: \quad D y^{2} \quad=d^{2}+B^{2} x
\end{aligned}
$$

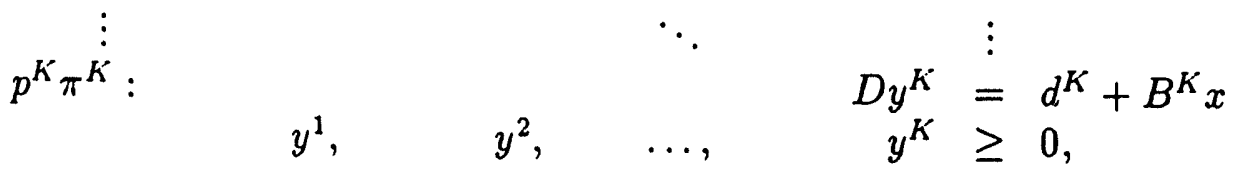

and to the dual linear problem (2.5):

$$
\begin{aligned}
& \begin{array}{rl}
\max & z_{D} \\
p^{1} \pi^{1} & \left(d^{1}+B^{1} x\right)+p^{2} \pi^{2}\left(d^{2}+B^{2} x\right)+\cdots+p^{K} \pi^{K}\left(d^{K}+B^{K} x\right) \\
\pi^{1} & D
\end{array} \\
& \pi^{2} \quad D \\
& \begin{array}{l}
\leq f \\
\leq f
\end{array} \\
& \pi^{K} \quad D \\
& \leq f
\end{aligned}
$$

The primal problem is parameterized in the right-hand side by $x$. The assumption of (2.2) being finite implies that (2.4) is finite for at least one value of $x$ for which $x \geq 0$ and $A x=b$. Applying the Duality Theorem of Linear Programming we state that (2.5) has to be feasible. The feasibility conditions

$$
\pi^{\omega} D-f \leq 0
$$

indicate that the feasible region $\left\{\pi^{\omega} \mid \pi^{\omega} D-f \leq 0\right\}$ is independent of $x$ and $\omega$ and is simply repeated for each scenario $\omega \in \Omega$.

The assumption of (2.2) being feasible requires feasibility of the primal problem (2.4) for at least one value of $x$ satisfying $x \geq 0$ and $A x=b$. We define $\pi:=$ $\left(\pi^{1}, \pi^{2}, \ldots, \pi^{K}\right)$ to be the vector of dual variables of problem (2.5). By the Duality Theorem again, $z_{D}$ in (2.5) has to be finite. Let $\pi^{j}, j=1, \ldots, p$ be the extreme points 
and $\pi^{j}, j=p+1, \ldots, p+q$ be representatives of the extreme rays of the feasible region of (2.5), where $\pi^{j}:=\left(\pi^{1 j}, \pi^{2 j}, \ldots, \pi^{K j}\right)$. Problem (2.5) is finite if and only if

$$
\pi^{\omega j}\left(d^{\omega}+B^{\omega} x\right) \leq 0, \quad j=p+1, \ldots, p+q, \omega \in \Omega .
$$

Constraints (2.7) may le appended to problem (2.3) to ensure that the dual problem (2.5) is bounded.

Next we outer linearize the infimal value function in (2.3), whose value is exactly

$$
\max _{j=1, \ldots, p} \sum_{\omega \in \Omega} p^{\omega} \pi^{\omega j}\left(d^{\omega}+B^{\omega} x\right) .
$$

By expressing the infimal value function by the outer linearized dual problem and using $\theta$ as the smallest upper bound, the problem can be represented in the following form:

$$
\begin{aligned}
\min Z=c x+\theta & \\
A x \quad & =b \\
x \quad & \geq 0 \\
\theta \geq \sum_{\omega \in \in \Omega} p^{\omega} \pi^{\omega j}\left(d^{\omega}+B^{\omega} x\right), & j=1, \ldots, p \\
\pi^{j}\left(d^{\omega}+B^{\omega} x\right) \leq 0, & j=p+1, \ldots, p+q, \omega \in \Omega .
\end{aligned}
$$

Relaxation is applied to solve problem (2.10) as we do not want to know all $\pi^{j}, j=$ $1, \ldots, p+q$ in advance. Given a solution $(\hat{x}, \hat{\theta})$ from the master problem, one solves problem (2.4) or problem (2.5), actually by solving the individual problems (2.11):

$$
\begin{aligned}
z^{\omega *}(\hat{x})=\min z_{P}^{\omega}=y^{\omega} & \\
D y^{\omega} & =d^{\omega}+B^{\omega} x \\
y^{\omega} & \geq 0, \quad \omega \in \Omega,
\end{aligned}
$$

or the dual problems $(2.12)$ of these:

$$
\begin{aligned}
z^{\omega *}(\hat{x})=\max z_{D}^{\omega}= & \pi^{\omega}\left(d^{\omega}+B^{\omega} \hat{x}\right) \leq f, \quad \omega \in \Omega . \\
& \pi^{\omega} D
\end{aligned}
$$

We call $\pi^{\omega *}(\hat{x})$ the optimum dual solution vector. If primal infeasibility or dual unboundedness is detected, with $\pi^{\omega^{\circ}}(\hat{x})$ denoting the corresponding extreme ray, a feasibility cut

$$
\pi^{\omega^{0}}(\hat{x}) \cdot\left(d^{\omega}+B^{\omega} x\right) \leq 0
$$

is added to the master problem. If all primal problems are feasible or all dual problems bounded, an optimality cut

$$
\theta \geq \sum_{\omega \in \Omega} p^{\omega} \pi^{\omega *}(\hat{x}) \cdot\left(d^{\omega}+b^{\omega} x\right)
$$

is added to the master problem. We call

$$
L(\hat{x}, x):=\sum_{\omega \in \Omega} p^{\omega} \pi^{\omega *}(\hat{x}) \cdot\left(d^{\omega}+B^{\omega} x\right)
$$


an outer linearization of the second-stage costs, which are defined by

$$
z(\hat{x}):=\sum_{\omega \in \Omega} z^{\omega *}(\hat{x})
$$

The relation

$$
L(\hat{x}, x) \leq z(x)
$$

formulates the main property of the outer linearization. Any cut regardless of the $\hat{x}$ from which it was originally derived is a valid cut as long as it does not violate the main property of outer linearization.

Benders decomposition provides upper and lower bounds to the solution in each iteration. In the $l$-th iteration,

$$
\mathrm{LB}^{l}:=c \hat{x}^{l}+\hat{\theta}^{l}
$$

with $\hat{x}^{l}, \hat{\theta}^{l}$ being the optimum solution of the master problem in iteration $l$ is defined to be a lower bound, and

$$
\mathrm{UB}^{l}:=\min \left\{\mathrm{UB}^{l-1}, c \hat{x}^{l}+z\left(\hat{x}^{l}\right)\right\}, \quad \mathrm{UB}^{0}=\infty
$$

with $z\left(\hat{x}^{l}\right)$ the second-stage costs associated with the solution $\hat{x}^{l}$ of the master problem, to be an upper bound to the solution of the problem. If

$$
\left(\mathrm{UB}^{l}-\mathrm{LB}^{l}\right) / \mathrm{LB}^{l} \leq \mathrm{TOL}
$$

where TOL is a given tolerance, the problem is said to be solved with a sufficient accuracy.

Summarizing, Benders decomposition splits the original problem into a master problem and a subproblem, which in turn decomposes into a series of independent subproblems, one for each $\omega \in \Omega$. The latter are used to generate cuts. The master problem, the subproblems and the cuts are summarized in (2.21), (2.22) and (2.23). The master problem:

$$
\begin{aligned}
\min z_{M}= & c x+\theta \\
\mathrm{s} / \mathrm{t} & =b \\
A x & \geq g^{l}, l=1, \ldots, L \\
-G^{l} x+\alpha^{l} \theta & \geq 0 \\
x, \quad \theta & \geq 0
\end{aligned}
$$

The sub problems:

$$
\begin{aligned}
\min z^{\omega}=p^{\omega} f y^{\omega} & \\
\text { s/t } p^{\omega} \pi^{\omega}: & =d^{\omega}+B^{\omega} x \\
y^{\omega} & \geq 0, \omega \in \Omega, \text { hboxe.g. } \Omega=\{1,2, \ldots, K\}
\end{aligned}
$$

where $p^{\omega} \pi^{\omega *}$ is the optimal dual solution of subproblem $\omega$. 
The cuts:

$$
\begin{aligned}
g=\sum_{\omega} p^{\omega} \pi^{\omega *} d^{\omega} & =E\left(\pi^{\omega *} d^{\omega}\right) \\
G=\sum_{\omega} p^{\omega} \pi^{\omega *} B^{\omega} & =E\left(\pi^{\omega *} B^{\omega}\right) \\
\alpha^{l}=0 \text { for a feasibility cut, } \alpha^{l} & =1 \text { for a optimality cut. }
\end{aligned}
$$

By solving the master problem (2.21), where cuts are initially absent and then sequentially added, we obtain a t,rial solution $\hat{x}$. Given $\hat{x}$ we can scive $\tilde{K}$ subproblems $\omega \in \Omega(2.22)$ to compute a cut (2.23). The cut is a lower bolid on the expected value of the second-stage costs represented as a function of $x$. '́uts are sequentially added to the master problem and new values of $\hat{x}$ are obtained until the optimality criterion is met. We distinguish between two types of cuts: feasibility cuts and optimality cuts. The first type refers to infeasible subproblems for a given $\hat{x}$ and the latter to feasible and optimum subproblems, given $\hat{x}$.

If the expected values $z, G$, and $g$ are computed exactly, that is, by evaluating all scenarios $\omega \in \Omega$, we refer to $i$ ' as the universe case. As $w \in$ will see later the number of scenarios easily gets out of hand and it is not always possible to solve the universe case. Therefore methods are songht that guarantee a satisfactory solution without having to solve the universe case. We employ Monte Carlo sampling techniques to obtain accurate estimates of the expected values $z, G$, and $g$.

\subsection{MONTE CARLO SAMPLING}

\subsubsection{Multidimensional Integration}

The computation of the expected future costs $z$, the coefficients $G$ and the right-hand side $g$ of the cuts requires the computation of multiple integrals or multiple sums. The expected value of the second-stage costs, e.g. $z=E z^{\omega}=E(C)$, is an expectation of functions $C\left(v^{\omega}\right), \omega \in \Omega$, where $C\left(v^{\omega}\right)$ is obtained by solving a linear program. $V$ (in general) is an $h$-dimensional rundom vector parameter, $V=\left(V_{1}, \ldots, V_{h}\right)$, with outcomes $v^{\omega}=\left(v_{1}, \ldots, v_{h}\right)^{\omega}$. For example, in expansion planning problems of electric power systems, $V_{i}$ represents the percent of generators of type $i$ down for repair or transmission lines of type $i$ not operating, and $v_{i}^{\omega}$ is the observed $i$ andom percent outcome. In portfolio management problems, $V_{i}$ might represent the random value of the independent factor $i$ used to describe the random value of returns of the universe of assets in a portfolio, and $v_{i}^{\omega}$ the observed outcome. The vector $v^{\omega}$ is also denoted by $v$, and $p\left(v^{\omega}\right)$ alias $p(v)$ denotes the corresponding probability. $\Omega$ is the set of all possible random events and is constructed by crossing the sets of outcomes: $\Omega=$ $\Omega_{1} \times \Omega_{2} \times \cdots \times \Omega_{h}$. We assume independence of the stochastic parameters $V_{1}, \ldots, V_{h}$. With $P$ being the probability measure, the integral $E C(V)={ }_{\int} C\left(v^{\omega}\right) P(d \omega)$ takes the form of a multiple integral $E C(V)=\int \cdots \int C(v) p(v) d v_{1} \cdots d v_{h}$, or, in the case of discrete distributions, the form of a multiple sum $E C(V)=\sum_{v_{1}} \cdots \sum_{v_{h}} C(v) p(v)$. Based on the independence assumption, $p(v)=p_{1}\left(v_{1}\right) \cdots p_{h}\left(v_{h}\right)$. 
In the following discussion we concentrate on discrete distributions. This is not a restriction as the approach can be easily adapted for continuous distributions. In practical applications we may assume that all distributions can be approximated with sufficient accuracy by discrete ones. In practical problems the number of terms in the multiple sum computation becomes astronomically large and therefore the evaluation of multiple sums by direct summation is not practical. This is especially true because the evaluation of each term in the multiple sum requires the solution of a linear program. For example, in order to compute the expected values of the subproblem costs, the coefficients and right-hand sides of the cuts, a linear program has to be solved for each outcome $\omega \in \Omega$.

The expected value of the subproblem costs is denoted by

$$
z=E C\left(v^{\omega}\right)=E f y^{* \omega}, \quad \omega \in \Omega
$$

with $y^{* \omega}$ being the optimum solution of subproblem $\omega$. The number of elements of $\Omega$ is determined by the dimensionality of the stochastic vector $V=\left(V_{1}, \ldots, V_{h}\right)$. Typically the dimension $h$ of $V$ is quite large.

For example, in expansion planning problems of electric power systems, one component of $V$ denotes the availability of one type of generator or one demand of power in a node of a multi-area supply network, or the availability of one type of transmission line connecting two nodes. Consider several nodes and arcs and orie demand and some options of generators at each node. The number of scenarios $K$ in the universe case quickly gets out of hand, even if the distribution of each component of $V$ is determined by just a small number $K^{i}$ of discrete points. Suppose e.g. that $h=20$ and $K^{i}=2, i=1, \ldots, 20$. This refers to the case that 20 components of the system are either in operation or down for repair. The resulting number of scenarios is as large as $2^{20} \approx 10^{6}$. Alternatively, suppose that $h=20$ and $K^{i}=5, i=1, \ldots, 20$. Then the total number of terms in the expected value calculations is $K=5^{20} \approx 10^{14}$, which is not practically solvable by direct summation.

In portfolio management problems, one component of $V$ may denote the value of an orthogonal factor. Usually up to 80 factors are used to describe the random outcomes of up to severai thousand assets. Suppose, for example, we used 80 factors, and discrete stochastic parameters with only 3 outcomes each. Then the number of possible scenarios would be as much as $3^{80} \approx 10^{38}$, an astronomically large number.

Monte Carlo methods appear promising for the computation of multiple integrals or multiple sums for $h$ large (Davis and Rabinowitz (1984) [32]). See Hammersly and Handscomb (1964) [59] for a description of Monte Carlo sampling techniques.

\subsubsection{Crude Monte Carlo}

Suppose $v^{\omega}, \omega=1, \ldots, N$, are scenarios sampled independently from their joint probability mass function. Then $C^{\omega}=C\left(v^{\omega}\right)$ are independent random variates with 
expectation $z$.

$$
\bar{z}=(1 / N) \sum_{\omega=1}^{N} C^{\omega}
$$

is an unbiased estimator of $z$, and its variance is

$$
\sigma_{\bar{z}}^{2}=\sigma^{2} / N
$$

where $\sigma^{2}=\operatorname{var}(C(V))$. Thus the standard error decreases with sample size $N$ by $N^{-0.5}$. The convergence rate of $\bar{z}$ to $z$ is independent of the dimension $h$ of the random vector $V$.

\subsubsection{Importance Sampling}

We rewrite

$$
z=\sum_{\omega \in \Omega} C\left(v^{\omega}\right) p\left(v^{\omega}\right)=\sum_{\omega \in \Omega} \frac{C\left(v^{\omega}\right) p\left(v^{\omega}\right) q\left(v^{\omega}\right)}{q\left(v^{\omega}\right)}
$$

by introducing a probability mass function $q\left(v^{\omega}\right)$. We can view $q$ as a probability mass function of a random vector $W$ that assumes the same outcomes as $V$ but with different probabilities. Therefore by change of variables,

$$
z=E \frac{C(W) p(W)}{q(W)}
$$

We obtain a new estimator of $z$,

$$
\bar{z}=\frac{1}{N} \sum_{\omega=1}^{N} \frac{C\left(w^{\omega}\right) p\left(w^{\omega}\right)}{q\left(w^{\omega}\right)}
$$

which has a variance of

$$
\operatorname{var}(\bar{z})=\frac{1}{N} \sum_{\omega \in \Omega}\left(\frac{C\left(w^{\omega}\right) p\left(w^{\omega}\right)}{q\left(w^{\omega}\right)}-z\right)^{2} q\left(w^{\omega}\right)
$$

Choosing $q^{*}\left(w^{\omega}\right)=C\left(w^{\omega}\right) p\left(w^{\omega}\right) / \sum_{\omega \in \Omega} C\left(w^{\omega}\right) p\left(w^{\omega}\right)$ would lead to $\operatorname{var}(\bar{z})=0$, which means we could get a perfect estimate of the multiple sum just by one single observation. However this is practically useless, since to sample $C p / q$ we have to know $q$ and to determine $q$ we need to know $z=\sum_{\omega \in \Omega} C\left(w^{\omega}\right) p\left(w^{\omega}\right)$, which we eventually want to compute. Nevertheless this result helps to derive heuristics of how to choose $q$ : it should be approximately proportional to the product $C\left(w^{\omega}\right) p\left(w^{\omega}\right)$ and have a form that can be integrated analytically. For instance, using the additive (separable in the components of the stochastic vector) approximation of (2.32),

$$
C(V) \approx \sum_{i=1}^{h} C_{i}\left(V_{i}\right)
$$


could be a possible way to compute a proper $q$ :

$$
q\left(w^{\omega}\right) \approx \frac{C\left(w^{\omega}\right) p\left(w^{\omega}\right)}{\sum_{i=1}^{h} \sum_{\omega \in \Omega_{i}} C_{i}\left(w^{\omega}\right) p_{i}\left(w^{\omega}\right)} .
$$

In this case one has to evaluate only $n 1$-dimensional sums instead of $1 \mathrm{~h}$-dimensional sum. Depending on how well the additive model approximates the original cost surface, the above-mentioned estimator will lead to smaller variances compared to crude Monte Carlo sampling. Of course, if the original cost surface has the property of additivity (separability), no sampling is required, as the multiple sum is computed exactly by $h 1$-dimensional sums.

The advantage of this approach lies in the fact that even if the additive model is a poor approximation to the cost surface, the method still works. The price that has to be paid is a high sample size. The variance reduction compared to crude Monte Carlo will be small. For the theory of importance sampling we refer to Glynn and Iglehart (1989) [58]. See also Dantzig and Glynn (1990) [24].

R. Entriken and M. Nakayama in Dantzig et al. (1989) [25] developed an importance sampling scheme using an additive model to approximate the cost function $E C(V)$. In particular, $C(v)$ is approximated by a marginal cost model, considering marginal costs in each dimension $i$ of $V$ and a base case, the point from which the $a_{j}$ ? noximation is developed. We will use this approach here. As we employ importance sampling within the Benders decomposition algorithm, the costs $C(v, \hat{x})$, the approximation of the costs $\Gamma(v, \hat{x})$ and thus the importance distribution of $q(v, \hat{x})$ depend also on $\hat{x}$, the current solution of the master problem. Introducing the costs of the base case $C(\tau, \hat{x})$ makes the model more sensitive to the impact of the stochastic variables $V$ :

$$
\begin{gathered}
C(V, \hat{x}) \approx \Gamma(V, \hat{x})=C(\tau, \hat{x})+\sum_{i=1}^{h} M_{i}\left(V_{i}, \hat{x}\right), \\
M_{i}\left(V_{i}, \hat{x}\right)=C\left(\tau_{1}, \ldots, \tau_{i-1}, V_{i}, \tau_{i+1}, \ldots, \tau_{h}, \hat{x}\right)-C(\tau, \hat{x}) .
\end{gathered}
$$

The vector $\tau=\left(\tau_{1}, \ldots, \tau_{h}\right)$ can be any arbitrarily chosen point out of the set of values $v_{i}, i=1, \ldots, h$. For example we choose $\tau_{i}$ as the outcome of $V_{i}$ that leads to the lowest costs. Figure 2.1 represents schematically the true cost function $C(V)$ and the additive approximation function $\Gamma(V)$ for the case of only two dimensions.

Note that the second-stage costs are computed by a linear program, where the uncertain parameters appear in the right-hand side. Therefore the second-stage costs are convex in the stochastic parameters $V$. In the most general case of a convex function $C\left(V_{1}, \ldots, V_{h}, \hat{x}\right)$ the choice of the base case $\tau=\left(\tau_{1}, \ldots, \tau_{h}\right)$ to be these values of $\left(V_{1}, \ldots, V_{h}\right)$ that minimize the function $C\left(V_{1}, \ldots, V_{h}, \hat{x}\right)$ for given $\hat{x}$, requires the solution of a convex minimization problem (with discrete variables $V$ ). This is impractical, and another choice of a base case is taken. In many cases the base case of lowest costs can be found easily. For example, in the context of expansion planning of power systems, choosing the base case of lowest costs means selecting respectively lowest demands and highest availabilities of generators and transmission lines. 


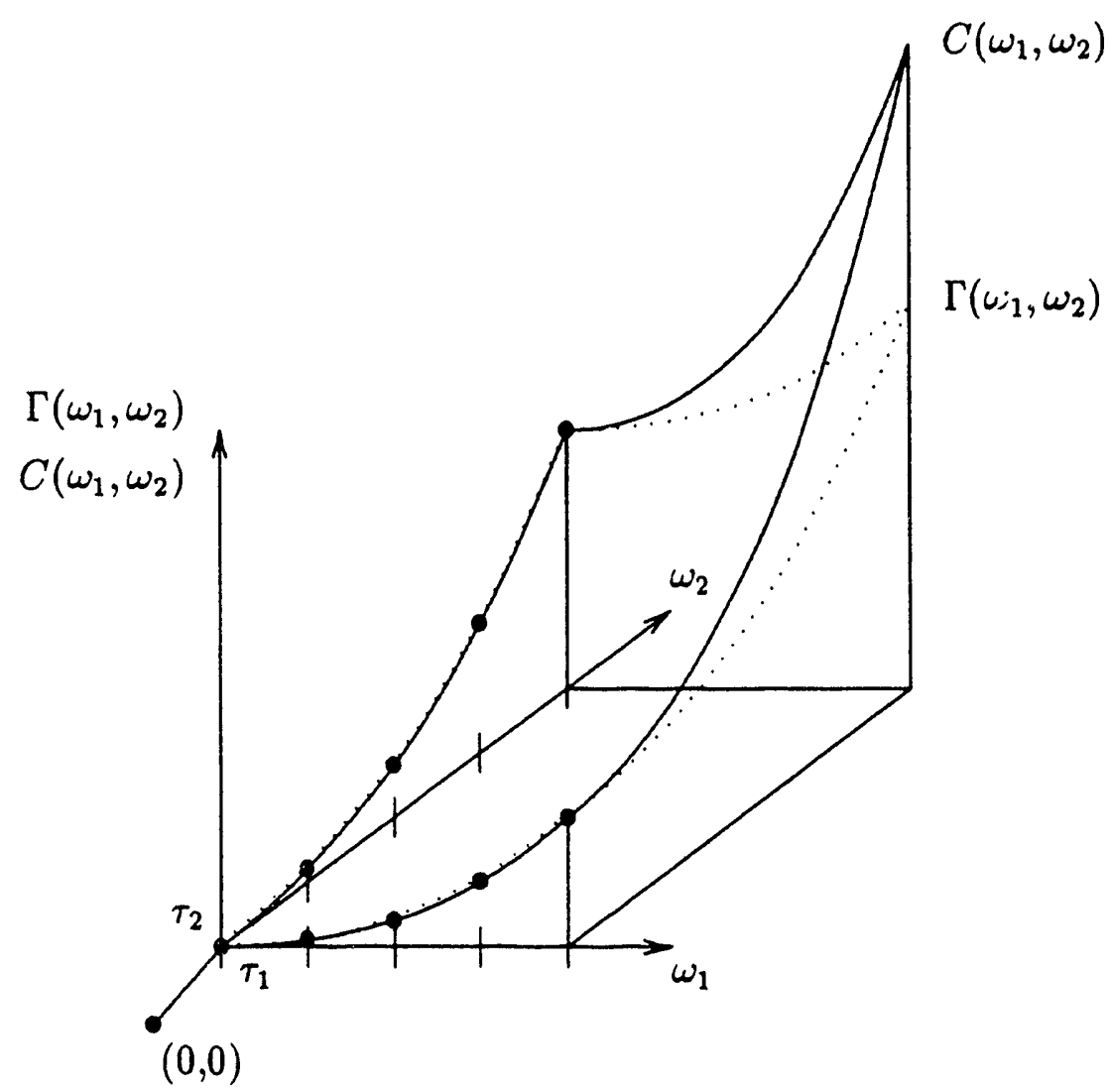

Figure 2.1: Additive approximation versus true cost function

Defining

$$
\bar{M}_{i}(\hat{x})=E M_{i}\left(V_{i}, \hat{x}\right)=\sum_{\omega \in \Omega_{i}} M_{i}\left(v_{i}^{\omega}, \hat{x}\right) p\left(v_{i}^{\omega}\right)
$$

and

$$
F\left(v^{\omega}, \hat{x}\right)=\frac{C\left(v^{\omega}, \hat{x}\right)-C(\tau, \hat{x})}{\sum_{i=1}^{h} M_{i}\left(v_{i}^{\omega}, \hat{x}\right)}
$$

where we assume that $\sum_{i=1}^{h} M_{i}\left(v_{i}^{\omega}, \hat{x}\right)>0$, so that at least one $M_{i}\left(v_{i}^{\omega}, \hat{x}\right)>0$, we can express the expected value of the costs in the following form:

$$
z(\hat{x})=C(\tau, \hat{x})+\sum_{i=1}^{h} \bar{M}_{i}(\hat{x}) \sum_{\omega \in \Omega} F\left(v^{\omega}, \hat{x}\right) \frac{M_{i}\left(v_{i}^{\omega}, \hat{x}\right)}{\bar{M}_{i}(\hat{x})} \prod_{j=1}^{h} p_{j}\left(v_{j}^{\omega}\right)
$$

Note that this formulation consists of a constant term and a sum of $h$ expectations. Given a fixed sample size $N$ we partition $N$ into $h$ sub-samples, with sample sizes $N_{i}$, 
$i=1, \ldots, h$ such that $\Sigma N_{i}=N$ and $N_{i} \geq 1, i=1, \ldots, N$ where $N_{i}$ is approximately proportional to $\bar{M}_{i}$. The $h$ expectations are separately approximated by sampling marginal densities. The $i$-th expectation corresponds of course to the $i$-th component of $V$. Generating sample points in the $i$-th expectation we use the importance density $\left(p_{i} M_{i} / \bar{M}_{i}\right)$ for sampling the $i$-th component of $V$ and the original marginal densities for any other components. Denoting

$$
\mu_{i}(\hat{x})=\frac{1}{N_{i}} \sum_{j=1}^{N_{i}} F\left(v^{j}, \hat{x}\right)
$$

the estimate of the $i$-th sum, we obtain

$$
\bar{z}(\hat{x})=C(\tau, \hat{x})+\sum_{i=1}^{h} \bar{M}_{i}(\hat{x}) \mu_{i}(\hat{x}),
$$

the estimated expected value of the second-stage costs.

Let $\bar{\sigma}_{i}^{2}(\hat{x})$ be the sample variance of the $i$-th expectation, where $\sigma_{i}^{2}(\hat{x})=0$ if $N_{i}=1$. The estimated variance of the mean, $\sigma_{\bar{z}}^{2}(\hat{x})$, is then given by

$$
\sigma_{\bar{z}}^{2}(\hat{x})=\sum_{i=1}^{h} \frac{\bar{M}_{i}^{2}(\hat{x}) \bar{\sigma}_{i}^{2}(\hat{x})}{N_{i}} .
$$

Using importance sampling one can achieve significant variance reduction. The experiment of M. Nakayama in Dantzig et al. (1989) [25] claims a variance reduction of 1:20000 using importance sampling versus crude Monte Carlo sampling. For a given optimal $\hat{x}$ the second-stage costs of a multi-area expansion planning model with 192 universe scenarios were sampled with a sample size of 10 using both methods and the results compared.

The derivation above concerned the estimation of the expected second-stage costs $z(\hat{x})$. To derive a cut we use an analogous framework. Note that a cut is defined as an outer linearization of the second-stage costs represented as a function of $x$, the first stage variables. At $\hat{x}$, the value of the cut is exactly the expected second-stage costs $z(\hat{x})$. Note also that any choice of $q$ is a valid choice and leads to an unbiased estimate. As we do not want to derive different importance distributions for the coefficients and the right-hand side of a cut, we use the $q$ already at hand from the cost estimation. Therefore we employ directly the cost approximation scheme and the importance distribution to compute the gradient and the right-hand side of a cut. With $B\left(v^{\omega}\right):=B^{\omega}$ and $d\left(v^{\omega}\right):=d^{\omega}$ being the outcome of $B$ and $d$ in scenarios $\omega \in \Omega$ and $\pi^{*}\left(v^{\omega}, \hat{x}\right):=\pi^{\omega *}(\hat{x})$, the optimum dual solution in scenario $\omega$, we define

$$
\begin{aligned}
F^{G}\left(v^{\omega}, \hat{x}\right) & =\frac{\pi^{*}\left(v^{\omega}, \hat{x}\right) B\left(v^{\omega}\right)-\pi^{*}(\tau, \hat{x}) B(\tau)}{\sum_{i=1}^{h} M_{i}\left(v_{i}^{\omega}, \hat{x}\right)}, \\
F^{g}\left(v^{\omega}, \hat{x}\right) & =\frac{\pi^{*}\left(v^{\omega}, \hat{x}\right) d\left(v^{\omega}\right)-\pi^{*}(\tau, \hat{x}) d(\tau)}{\sum_{i=1}^{h} M_{i}\left(v_{i}^{\omega}, \hat{x}\right)}
\end{aligned}
$$


and compute

$$
\begin{aligned}
G(\hat{x}) & =\pi^{*}(\tau, \hat{x}) B(\tau)+\sum_{i=1}^{h} \bar{M}_{i}(\hat{x}) \sum_{\omega \in \Omega} F^{G}\left(v^{\omega}, \hat{x}\right) \frac{M_{i}\left(v_{i}^{\omega}, \hat{x}\right)}{\bar{M}_{i}(\hat{x})} \prod_{j=1}^{h} p_{j}\left(v_{j}^{\omega}\right) \\
g(\hat{x}) & \left.=\pi^{*}(\tau, \hat{x}) d(\tau)+\sum_{i=1}^{h} \bar{M}_{i}(\hat{x}) \sum_{\omega \in \Omega} F^{g}\left(v^{\omega}, \hat{x}\right) \frac{M_{i}\left(v_{i}^{\omega}\right)}{\bar{M}_{i}(\hat{x})}\right) \prod_{j=1}^{h} p_{j}\left(v_{j}^{\omega}\right)
\end{aligned}
$$

the coefficients and the right-hand side of a cut. We estimate the expected values again by sampling using the sample points at hand from the computation of $\bar{z}$.

Using Monte Carlo sampling we obtain $\bar{z}(\hat{x}), \bar{G}(\hat{x}), \bar{g}(\hat{x})$, which are approximations of the expected values $z(\hat{x}), G(\hat{x}), g(\hat{x})$. We also obtain the estimated variance of the mean of the second-stage costs $\sigma_{\bar{z}}(\hat{x})$. The impact of using approximations instead of the exact parameters on the Benders decomposition algorithm is analyzed in the following section.

\subsection{PROBABILISTIC CUTS}

\subsubsection{The Influence of the Estimation Error}

Employing Monte Carlo sampling techniques means not solving all problems $\omega \in \Omega$, but solving problems $\omega \in S, S$ being a subset of $\Omega$. Instead of the exact expected values $z(\hat{x}), G(\hat{x}), g(\hat{x})$ we compute the estimates $\bar{z}(\hat{x}), \bar{G}(\hat{x}), \bar{g}(\hat{x})$ by importance sampling. We also estimate the error of the estimation of $z(\hat{x})$ by the variance $\operatorname{var}(\bar{z}(\hat{x}))=\sigma_{\bar{z}}^{2}(\hat{x})$. Thus for example in the case of the second-stage costs the estimation results in an estimated mean with some error distribution. There is good reason to assume that the error is normally distributed (Davis and Rabinowitz (1984) $[32])$. We define $\tilde{z}(\hat{x})$ to be normally distributed with mean $\bar{z}(\hat{x})$ and variance $\sigma_{\bar{z}}^{2}(\hat{x})$ :

$$
\tilde{z}(\hat{x}):=N\left(\bar{z}(\hat{x}), c_{\bar{z}}^{2}(\hat{x})\right)
$$

A cut obtained by sampling differs in general from a cut computed by solving the universe scenarios. The outer linearizations

$$
L(\hat{x}, x)=G(\hat{x}) x+g(\hat{x})
$$

with respect to the universe case, and

$$
\bar{L}(\hat{x}, x)=\bar{G}(\hat{x}) x+\bar{g}(\hat{x})
$$

with respect to the estimation, differ in the gradient and the right-hand side. At $x=\hat{x}$, the value at which the cut was derived, $L(\hat{x}, \hat{x})=z(\hat{x})$ and $\bar{L}(\hat{x}, \hat{x})=\bar{z}(\hat{x})$.

Thus if a true cut obtained by solving the universe case is binding at the solution $x=\hat{x}$, the variable $\theta$ takes on the value

$$
\theta=L(\hat{x}, \hat{x})=z(\hat{x})
$$


In the case of using Monte Carlo sampling we assign $\theta$ to be the value of the estimated costs at $\hat{x}, \bar{L}(\hat{x}, \hat{x})=\bar{z}(\hat{x})$, and correct for the estimation error at the right-hand side. Thus

$$
\theta=\bar{L}(\hat{x}, \hat{x})-\bar{z}(\hat{x})+z(\hat{x})
$$

which we write as

$$
\theta=\bar{L}(\hat{x}, \hat{x})+\epsilon(\hat{x}) .
$$

Equation (2.50) represents a valid statement for a solution $x=\hat{x}$. The correction term $\epsilon(\hat{x})=z(\hat{x})-\bar{z}(\hat{x})$ corrects for the estimation error. Of course, we do not know the difference $z(\hat{x})-\bar{z}(\hat{x})$ explicitly for each cut when we compute it. However we can obtain an estimate of the distribution of $\epsilon(\hat{x})$ by the estimation process. Using Monte Carlo importance sampling we obtain an unbiased estimate of $z(\hat{x}), \bar{z}(\hat{x})$, with variance $\sigma_{\bar{z}}^{2}(\hat{x})$. Thus $\epsilon(\hat{x})$ is normally distributed with mean 0 and variance $\sigma_{\bar{z}}^{2}(\hat{x})$

$$
\epsilon(\hat{x}):=N\left(0, \sigma_{\bar{z}}^{2}(\hat{x})\right) .
$$

Suppose next that a cut $\bar{L}(\hat{x}, x)=\bar{G}(\hat{x}) x+\bar{g}(\hat{x})$ was computed at $x=\hat{x}$, but is binding at a solution $\hat{\hat{x}}$ where $\hat{x} \neq \hat{\hat{x}}$. Applying the correction for the estimation error resulting from using Monte Carlo sampling instead of computing the expectations exactly, we obtain

$$
\theta=\bar{L}(\hat{x}, \hat{\hat{x}})+\epsilon(\hat{\hat{x}}) \text {. }
$$

The correction term for the estimation error is clearly the true value of the cut at $x=\hat{\hat{x}}$, obtained by solving the universe case, minus the value obtained by the sampling procedure:

$$
\epsilon(\hat{\hat{x}})=L(\hat{x}, \hat{\hat{x}})-\bar{L}(\hat{x}, \hat{\hat{x}}) .
$$

Again we do not know the difference $L(\hat{x}, \hat{\hat{x}})-\bar{L}(\hat{x}, \hat{\hat{x}})$ when we compute the cut. Also the distribution of the estimation error $\epsilon(\hat{\hat{x}})$ at $x=\hat{\hat{x}}$ is not known directly by the estimation procedure but can be computed. Thus, initially, and sufficiently for all practical purposes, we assume that

$$
\epsilon(\hat{\hat{x}}) \approx \epsilon(\hat{x}) \text { for } \hat{\hat{x}} \approx \hat{x} .
$$

This implies that the error distribution $\epsilon(x)$ is assumed to be approximately constant with respect to $x$. We will show empirically that this assumption is valid for most practical problems. Intuitively one can see it is valid because the value of $x$ changes only little before we terminate at the final solution. In general the set of observations $S$ is a sufficiently large subset, of $\Omega$ so that the variance $\sigma_{\bar{z}}^{2}$ is small.

Note that cuts computed by sampling do not necessarily meet the condition of outer linearization. They may intersect and separate parts of the feasible region of the second-stage problem. A sampled cut is therefore not necessarily a valid cut. The correction term $\epsilon(\hat{x})$, however, accounts for the error we make when using Monte Carlo sampling instead of solving the universe case. 


\subsubsection{The Estimation Error of a Cut as a Function of $x$}

Using Monte Carlo sampling we estimate the coefficients $G_{i}, i=1, \ldots, n$ and the right-hand side $g$ and obtain $\bar{G}$ and $\bar{g}$ by averaging $G^{\omega}=G_{1}^{\omega}, \ldots, G_{n}^{\omega}$ and $g^{\omega}$. For example, in the case of crude Monte Carlo sampling a sample of size $N$ would be obtained by sampling $G^{\omega}, g^{\omega}, \omega=1, \ldots, N$ from the original distribution $p(\omega)$, $\omega \in \Omega$ : , and an estimate of $G$ and $g$ would be computed by their means $\bar{G}, \bar{g}$. In the case of importance sampling a different and more complicated weighting scheme is used. We do not discuss this here and continue with the case of crude Monte Carlo.

For each sample $\omega, \omega=1, \ldots, N$, we obtain independent observations of the mutually dependent coefficients and the right-hand side of a cut:

$$
G_{1}^{\omega}, \ldots, G_{n}^{\omega}, g^{\omega} \text {. }
$$

Let us denote by $R$ the $n+1 \times N$ matrix composed of the observations of the coefficients and the right-hand side of the cut, subtracted by the mean of the observations:

$$
R=\left(\begin{array}{ccc}
G_{1}^{1}-\bar{G}_{1} & \cdots & G_{1}^{N}-\bar{G}_{1} \\
\vdots & & \vdots \\
G_{n}^{1}-\bar{G}_{n} & \cdots & G_{n}^{N}-\bar{G}_{n} \\
g^{1}-\bar{g} & \cdots & g^{N}-\bar{g}
\end{array}\right) .
$$

An estimate of the variance of the mean value of $\bar{L}(\hat{x}, x)=\bar{G}(\hat{x}) x+\bar{g}(\hat{x})$ can be obtained by

$$
\operatorname{var}(\bar{L}(\hat{x}, x))=\frac{1}{N} \frac{1}{N-1}\left(x^{T}, 1\right) R^{T} R\left(x^{T}, 1\right)^{T} .
$$

Defining

$$
y^{\omega}=R^{\omega}\left(x^{T}, 1\right)^{T}, \omega=1, \ldots, N,
$$

where $R^{\omega}$ refers to the column in $R$ that represents the observation $\omega$,

$$
R^{\omega}=\left(G_{1}^{\omega}-\bar{G}_{1}, \ldots, G_{n}^{\omega}-\bar{G}_{n}, g^{\omega}-\bar{g}\right),
$$

we can write the estimated variance of the value of the cut at $x$ as

$$
\operatorname{var}(\bar{L}(\hat{x}, x))=\frac{1}{N} \frac{1}{N-1} \sum_{\omega=1}^{N}\left(y^{\omega}\right)^{2} .
$$

This estimate can be easily computed. It follows that with knowledge of the matrix $R$ of observations of the coefficients and the right-hand side of a cut we are able to compute an estimate of the variance of the value of the cut, $\operatorname{var}(\bar{L}(\hat{x}, x))$, for any value of $x$. Clearly,

$$
\operatorname{var}(\tilde{L}(\hat{x}, \hat{x}))=\operatorname{var}(\bar{z}(\hat{x}))
$$

Using the estimation procedure we obtain an estimate of the distribution of the correction term:

$$
\epsilon(x)=N(0, \operatorname{var}(\bar{L}(\hat{x}, x)),
$$


where we also denote

$$
\sigma_{\bar{L}}^{2}(x):=\operatorname{var}(\bar{L}(\hat{x}, x))
$$

The error term $\epsilon(x)$ now correctly represents an estimate of the distribution of the difference $L(\hat{x}, x)-\bar{L}(\hat{x}, x)$ as a function of $x$.

While it seems to be impractical to store the matrix $R$ to obtain the error estimate of each cut as a function of that particular solution of the master problem $\hat{\hat{x}}$ at which the cut is binding, a Taylor approximation of $\operatorname{var}(\bar{L}(\hat{x}, x))$ appears to be sufficiently accurate.

Let $\alpha(\hat{x})$ be the vector of local derivatives at $\hat{x}$ :

$$
\alpha(\hat{x}):=\left[\frac{\partial}{\partial x}(\operatorname{var}(\bar{L}(\hat{x}, x)))\right]_{\hat{x}}
$$

where

$$
\frac{\partial}{\partial x_{i}}(\operatorname{var}(\bar{L}(\hat{x}, x)))=\frac{2(N-1)}{N}(\bar{G}(\hat{x}) x+\bar{g}(\hat{x})) \bar{G}_{i}
$$

Using a first order Taylor approximation we can write $\operatorname{var}(\bar{L}(\hat{x}, x))$ in the following form:

$$
\begin{aligned}
\sigma_{L}^{2}(x)=\operatorname{var}(\bar{L}(\hat{x}, x)) & \approx \operatorname{var}(\bar{L}(\hat{x}, \hat{x}))+\alpha(\hat{x})(x-\hat{x}) \\
& =\operatorname{var}(\bar{z}(\hat{x}))+\alpha(\hat{x})(x-\hat{x}) .
\end{aligned}
$$

The estimate of the variance $\operatorname{var}(\bar{L}(\hat{x}, x))$ as a function of $x$ is represented as a sum of two terms, the estimated variance of the second-stage costs at $\hat{x}, \operatorname{var}(\bar{z}(\hat{x}))$, plus the linear term that represents the change of the variance, if $x \neq \hat{x}$. The latter term is assumed to be small, as $\alpha(\hat{x})$ is small.

We will continue with the assumption that the error is approximately constant with respect to $x$. That means we see the error mainly concentrated in the right-hand side of the cut and we assign the constant error term $\epsilon(\hat{x})$ rather than the variable correction term $\epsilon(x)$ to the right-hand side. We then take

$$
-\bar{G} x+\theta \geq \bar{g}+\epsilon(\hat{x})
$$

to be approximately a valid cut. Note that we can expect in the final solution of the Benders decomposition procedure that cuts will be binding at an $x$ very close to the $\hat{x}$ where they were originally derived.

\subsubsection{Upper and Lower Bounds}

For random second-stage costs $\tilde{z}\left(\hat{x}^{l}\right)$ and random right-hand sides $\bar{g}^{l}+\epsilon^{l}, l=1, \ldots, L$ the upper and lower bounds of $t j a$ problem as provided by Benders decomposition algorithm are probabilistic. 


\section{The Upper Bounds}

$$
\tilde{\mathrm{UB}}^{l}:=c \hat{x}^{l}+\tilde{z}\left(\hat{x}^{l}\right), \quad l=1, \ldots, L
$$

are random parameters, normally distributed with means $\overline{U B}^{\prime}$ and variances $\sigma_{\bar{z}}\left(\hat{x}^{l}\right):$

$$
\tilde{\mathrm{UB}}^{l}:=N\left(\overline{\mathrm{UB}}^{l}, \sigma_{\bar{z}}^{2}\left(\hat{x}^{l}\right)\right) \quad l=1, \ldots, L .
$$

We define the lowest upper bound to be the upper bound with the lowest mean

$$
\tilde{U} \tilde{B}_{\min }^{L}:=\tilde{U} \tilde{B}_{\min }^{k}, k \in \arg \min \left\{\overline{U B}^{l}, l=1, \ldots, L\right\} .
$$

with corresponding variance $\sigma_{\mathrm{UB}_{\min }^{L}}^{2}$.

\section{The Lower Bounds}

The lower bounds are obtained from the solution of the master problem. To determine the distribution of a lower bound, consider the master problem at iteration $L$ :

$$
\begin{aligned}
& \tilde{\mathrm{LB}} \tilde{z}^{L}=\tilde{z}_{M}^{* L}=\min \tilde{z}_{M}^{L}=c x+\theta \\
& \mathrm{s} / \mathrm{t} \rho_{1}^{0}: \quad A x=b \\
& \rho^{1}: \quad-\bar{G}^{1} x+\theta \geq \bar{g}^{1}+\epsilon^{1} \\
& \rho^{L}: \quad-\bar{G}^{L} x+\theta \geq \bar{g}^{L}+\epsilon^{L} \\
& x, \quad \theta \geq 0 \text {, }
\end{aligned}
$$

where $L$ optimality cuts have been added to the originally relaxed master problem. We do not consider feasibility cuts in the following argument, as they are exact. The vector $\rho^{0}$ and the scalars $\rho^{l}, l=1, \ldots, L$ denote the dual prices. The right-hand sides $\bar{g}^{l}+\epsilon^{l}, l=1, \ldots, L$, are independent stochastic parameters, normally distributed. We assume independence as the cuts are generated from independent samples, and neglect the weak dependency due to $\hat{x}^{l}, l=1, \ldots, L$, via the Benders decomposition algorithm.

With the random parameters $\epsilon^{l}, l=1, \ldots, L$ in the right hand side the optimum solution $\tilde{z}_{M}^{*}$ will be random. One could experimentally obtain the distribution of $\tilde{z}_{M}^{* L}$ by randomly varying the right-hand sides according to $N$ samples $j=1, \ldots, N$ drawn from the normal distributions of $\epsilon^{l}, l=1, \ldots, L$ and by solving the master problem for all $N$ samples. One could estimate the mean and the variance of the distribution from the samples $j=1, \ldots, N$. As this is a very expensive way to obtain an estimate of the lower bound distribution, we proceed instead in the following way. We have already stated that we choose a sample size $|S|$ such that the variances $c_{\bar{z}}^{l}, l=1, \ldots, L$ are small. In this case we can then perform a local error analysis and assume that for all outcomes of the random right hand sides $\epsilon^{l}, l=1, \ldots, L$, the optimum solution of 
the master problem has the same basis. Then we can define the optimum solution of the master problem

$$
\tilde{z}_{M}^{* L}:=N\left(\bar{z}_{M}^{L}, \operatorname{var}\left(\tilde{z}_{M}^{* L}\right)\right)
$$

to be a random parameter, normally distributed with mean $\bar{z}_{M}^{L}$ and variance $\operatorname{var}\left(\tilde{z}_{M}^{* L}\right)$, and compute the mean of the lower bound estimate,

$$
\begin{aligned}
& \bar{z}_{M}^{* L}=\min z_{M}^{L}=c x+\theta \\
& \text { s/t } \rho_{1}^{0}: \quad A x=b \\
& \rho^{1}: \quad-\bar{G}^{1} x+\theta \geq \bar{g}^{1} \\
& \begin{aligned}
\vdots & \\
\rho^{L}: & -\bar{G}^{L} x+\theta \geq \bar{g}^{L}
\end{aligned} \\
& x, \quad \theta \geq 0 \text {, }
\end{aligned}
$$

by substituting the means $\bar{\epsilon}^{\prime}=0, l=1, \ldots, L$ for the random right-hand sides. We compute the variance $\operatorname{var}\left(\tilde{z}_{M}^{*}\right)$ by using the dual formulation of the master problem:

$$
\operatorname{var}\left(\tilde{z}_{M}^{*}\right)=\sum_{l=1}^{L}\left(\rho^{l}\right)^{2} \sigma_{\bar{z}}^{2}\left(\hat{x}^{l}\right)
$$

As the lower bound means increase monotonically with the number of iterations, we obtain the largest lower bound by

$$
\tilde{\mathrm{LB}}^{L}=\tilde{z}_{M}^{* L}
$$

which is normally distributed:

$$
\tilde{\mathrm{LB}}^{L}:=N\left(\overline{\mathrm{LB}}^{L}, \operatorname{var}\left(\tilde{\mathrm{LB}}^{L}\right)\right)
$$

The local error analysis can be easiiy extended to the case where we consider the error terms $\epsilon^{l}(x)$ as a function of the decision variables $x$ instead of constant. As before, we assume the variances $\sigma_{\bar{L}}^{2}(x)$ to be small so that for all outcomes of the random right-hand sides $\epsilon^{l}(x), l=1, \ldots, L$, the solution of the master problem has the same basis. We obtain the mean of the lower bound estimate by solving the master problem (2.74), with the means $\epsilon^{l}\left(x^{L}\right)=0, l=1, \ldots, L$ replacing the random terms in the right-hand side. Assuming the variance of the error terms $\epsilon^{l}\left(x^{L}\right)$ to be approximately constant in a small neighborhood of $x^{L}$, we obtain the variance $\operatorname{var}\left(\tilde{z}_{M}^{*}\right)$ by using the dual formulation of the master problem:

$$
\operatorname{var}\left(\tilde{z}_{M}^{*}\right)=\sum_{l=1}^{L}\left(\rho^{l}\right)^{2} \sigma_{L}^{2}\left(\hat{x}^{L}\right)
$$




\subsubsection{Stopping Rule}

By analogy to the deterministic Benders decomposition algorithm, we stop if the upper and lower bound are sufficiently close. In the case of probabilistic bounds, the algorithm has to be stopped if the upper and lower bound are indistinguishable in distribution. Using a student $t$-test we determine if $s^{l}>0$ with $95 \%$ probability, where

$$
s^{l}=\overline{\mathrm{UB}}^{l}-\overline{\mathrm{LB}}^{l}+\mathrm{TOL}
$$

and TOL being a given tolerance.

The employment of the student $t$-test requires independence of the upper and lower bound distributions. As independence is not ensured in the first place as an upper bound, and a binding cut in the master problem could be obtained from the same set of samples, we obtain independence by re-sampling the lowest upper bound before employing the student $t$-test. The $\hat{x}$ corresponding to the lowest upper bound and the corresponding importance distribution have to be stored. In each iteration we check if upper and lower bounds are close to each other, where we use the student $t$-test without fulfilling the independence requirement. If the bounds are close, we use new samples to compute an independent upper bound. Now we check if $s^{l}>0$ by the t-test.

\subsubsection{Confidence Interval}

After passing the student $t$-test in the last iteration, which means that the upper and lower bound means are indistinguishable, we obtain the optimum solution $\hat{x}^{L}, \hat{\theta}$ from the master problem. We derive from the distributions $\tilde{L B}^{L}$ and $\tilde{U B}^{L}$ a $95 \%$ confidence interval: on the left side by using the lower bound distribution and on the right side by using the upper bound distribution. We define

$$
C_{\text {left }}=1.96 \sqrt{\operatorname{var}\left(\tilde{\mathrm{LB}}{ }^{L}\right)}, \quad C_{\text {right }}=1.96 \sqrt{\operatorname{var}\left(\tilde{\mathrm{UB}}{ }^{L}\right)}
$$

and obtain the confidence interval

$$
\overline{\mathrm{LB}}-C_{\text {left }} \leq Z^{*} \leq \overline{\mathrm{UB}}+C_{\text {right }}
$$

for the final solution $Z^{*}$. If

$$
\left(C_{\text {left }}+C_{\text {right }}\right) / \overline{\mathrm{LB}}^{L} \leq C_{\text {tol }}
$$

where $C_{\text {tol }}$ is a predefined quality criterion for the confidence interval, the present solution is satisfactory. Otherwise the sample size has to be increased and the problem solved again. 


\subsubsection{Improvement of the Solution}

Suppose the solution with a certain sample size was not satisfying. Instead of starting from the beginning with an increased sample size we want to use the information, which we have already collected. To do this, we look for the binding cuts in the final solution, increase the sample size and recompute the binding cuts at the same $\hat{X}^{\ell}$, they were originally computed. This of course means that one has to store the values of $\hat{x}^{l}$ and the associated importance distributions or recompute the latter. The enlarged sample size leads to smaller variances of the binding cuts and eventually to a smaller confidence interval of the final solution. Berry-Esséen, e.g. Hall (1979), give upper bounds on the rates of convergence in the central limit theorem. Solving the master problem again with the improved binding cuts will in general not result in an intersection of the lower and upper bound. Therefore some more iterations are necessary to obtain the optimal solution according to the increased sample size. This improvement procedure could be employed iteratively until a satisfying solution is obtained. It is a possible way to improve a non satisfying solution. It may not be very efficient and there may be better ways to do so. In general we choose a sample size such that the obtained confidence interval is satisfying.

\subsection{THE ALGORITHM}

We can state now the algorithm as follows:

Step 0 Initialize:

$$
l=0, \overline{U B}^{0}=\infty .
$$

Step 1 Solve the relaxed master problem and obtain a lower bound:

$$
\overline{\mathrm{LB}}^{l}=c \hat{x}+\hat{\theta}^{l} \text {. }
$$

Step $2 l=l+1$.

Solve subproblems and obtain an upper bound:

$\overline{U B}^{l}=\min \left\{\overline{U B}^{l-1}, c \hat{x}^{l}+\bar{z}\left(\hat{x}^{l}\right)\right\}$. Compute and add a cut to the master problem, using Monte Carlo (importance) sampling.

Step 3 Solve the master problem and obtain a lower bound:

$\mathrm{LB}^{l}=c \bar{x}^{l}+\bar{\theta}^{l}$.

Step 4 Compute $s=\overline{\mathrm{UB}}^{l}-\overline{\mathrm{LB}}^{l}+$ TOL.

If $s>0$ (student $t$-test) go to Step 2 .

Step 5 Compute confidence interval and obtain a solution $Z^{*}, \hat{x}, \hat{\theta}$. Stop.

Improvement of the solution

Step 6 If $\left(C_{\text {left }}+C_{\text {right }}\right) / \mathrm{LB} \leq C_{\text {tol }}$, stop, otherwise go to Step 7 . 
Step 7 Increase the sample size and initialize $\overline{U B}^{0}=\infty$.

Step 8 Recompute binding cuts.

Upper bound: $\overline{U B}^{l}=\min \left\{\overline{U B}^{l-1}, C \hat{x}+\bar{z}\left(\hat{x}^{l}\right)\right.$.

Step 9 Go to step 3

\subsection{A CLASS OF MULTI-STAGE STOCHASTIC LINEAR PROGRAMS}

Large-scale deterministic mathematical programs, used for operations and strategic planning, are often dynamic linear programs. These problems have a staircase matrix structure. Multi-siuge stochastic linear programs are the stochastic extension of these programs. In general, the size of these programs can be extremly large because the number of scenarios grows exponentially with the number of periods. We will, however, address a certain restricted class in which the number of scenarios grows linearly with the number of stages (see Dantzig and Glynn (1990) [24] and Dantzig and Infanger (1991) [28]). The problem (whose constraints are stated below) breaks down into two parts: a deterministic dynamic part and a stochastic part. We call the deterministic part the master problem. It is a dynamic linear program with $T$ stages. The vectors $c_{t}$ and $b_{t}$, and the matrices $B_{t-1}$ and $A_{t}$, are assumed to be known with certainty.

$$
\begin{aligned}
& \min \sum_{t=1}^{T} c_{t} x_{t}+\sum_{t=1}^{T} E\left(f_{t} y_{t}^{\omega_{t}}\right) \\
& -B_{t-1} x_{t-1}+A_{t} x_{t} \quad=b_{t}, \quad t=1, \ldots, T, \quad B_{0}=0 \\
& -F_{t}^{\omega_{t}} x_{t}+\quad D_{t} y_{t}^{\omega_{t}}=d_{t}^{\omega_{t}}, \quad t=1, \ldots, T, \quad \omega_{t} \in \Omega_{t} \\
& x_{t}, \quad y_{t}^{\omega_{t}} \geq 0 \text {. }
\end{aligned}
$$

Each stage is associated with a stochastic subproblem. Uncertainty appears in the transition matrix $F_{t}^{\omega_{t}}$ and in the right-hand side vector $d_{t}^{\omega_{t}}$, where $\omega_{t}$ denotes an outcome of the stochastic parameters in period $t$, with $\Omega_{t}$ denoting the set of all possible outcomes in period $t$. The subproblems in each stage are assumed to be stochastically independent. The subproblem costs $f_{t}$ and the technology matrix $D_{t}$ are assumed to be deterministic parameters.

Facility expansion planning is an example of this type of formulation. The master problem models the expansion of the facilities over time. The decision variables are the capacity built and the capacity available at time $t$. The subproblems model the operation of these capacities in an uncertain environment. Take for exampla the case of expansion planning of power systems: The expansion or replacement of capacities of generators and transmission lines are determined in the master problem. The capacities at each period $t$ are made available to the system for operation. The subproblems model the power system operation, the optimal scheduling of the available cracities to meet the demand for electricity. The availabilities of generators and 
transmission lines and the demands are uncertain and not known at the time when the expansion decision is made.

The approach is primarily "here and now" (Dantzig and Madansky (.961) [29]) and justified by high investment ccsts and long lead-times for capacity expansion. However, as the operations subproblems are stochastically independent and only the expected operation cost rather than the state of the system after period $t$ affects the expansion plan (as failures of equipment get repaired, and uncertainty in the demands are interpreted as deviations from a demand path), "here and now" is equivalent to "wait and see". This means that the optimal decision in period $t+1$ depends only on the capital stock on hand at the start of period $t+1$ and is independent of any observed outcomes in period $t$; i.e., the same optimal capacity expansion decision would be made before and after period $t$ operations. Thus the facility expansion plan can be laid out at the beginning for the whole planning horizon based on the expansion corts and the expected operation costs. This permits the multi-stage problem to be treated as if it were a two-stage problem. The first "stage" concerns the single decision of what facility expansion there will be in all future periods without knowledge of the particular outcomes of the uncertainty parameters in future periods. The second "stage" concerns the operations problems, where the recourse decisions made depend on the realizations of the stochastic parameters. Note that for $T=1$, the problem is 'xactly a two stage stochastic linear program with recourse. For $T \geq 2$ the problem is a two "stage" problem with the second stage consisting of $T$ independent subproblems. Figure 2.2 represents he decision tree corresponding to the special class of "here and now" multi-stage projlems.

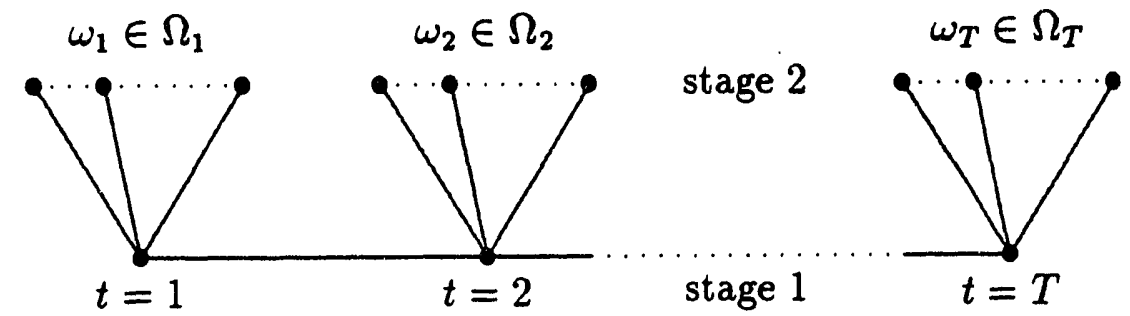

Figure 2.2: Decision tree for the special class of "here and now" multi-stage problems

Using Benders decomposition we decompose the special class of "here and now" multi-stage problems as follows: 


\section{The master problem}

$$
\begin{aligned}
z_{M}^{L}=\min \sum_{t=1}^{T} c_{t} x_{t}+\sum_{t=1}^{T} \theta_{t} & \\
-B_{t-1} x_{t-1}+A_{t} x_{t} & =b_{t}, \quad t=1, \ldots, T, \quad B_{0}=0 \\
-G_{t}^{l} x_{t}+\quad \alpha_{t}^{l} \theta_{t} & \geq g_{t}^{l}, \quad t=1, \ldots, T, \quad l=1, \ldots, L \\
x_{t} &
\end{aligned}
$$

where the latter constraints, called cuts, are initially absent but are added in later iterations. The master problem is optimized to obtain an approximate optimal feasible solution $x_{t}=\hat{x}_{t}^{l}$ that is used as input to the subproblems.

The subproblems for $\omega_{t}$ in period $t$

$$
\begin{array}{rlrl}
z_{t}^{\omega_{t}}\left(\hat{x}_{t}^{l}\right)=\min f_{t} y_{t}^{\omega_{t}} & & \\
\pi_{t}^{\omega_{t}}\left(\hat{x}_{t}^{l}\right):=y_{t}^{\omega_{t}} & =d_{t}^{\omega_{t}}+F_{t}^{\omega_{t}} \hat{x}_{t}^{l}, & & \omega_{t} \in \Omega_{t}, \quad t=1, \ldots, T \\
y_{t}^{\omega_{t}} & \geq 0, & \hat{x}_{t}^{l} \text { given, } &
\end{array}
$$

where $\pi_{t}^{\omega_{t}}=\pi_{t}^{\omega_{t}}\left(\hat{x}_{t}^{l}\right)$ are dual multipliers corresponding to the constraints and $z_{t}^{\omega_{t}}=$ $z_{t}^{\omega_{t}}\left(\hat{x}_{t}^{l}\right)$ is the value of the objective as a function of $\hat{x}_{t}^{l}$. These are used to generate the riext cut for the master problem.

\section{The cuts}

For $t=1,2, \ldots, T$,

$$
G_{t}^{l}=E\left(\pi^{\omega_{t}} B^{\omega_{t}}\right), \quad g_{t}^{l}=E\left(\pi^{\omega_{t}} d^{\omega_{t}}\right), \quad z_{t}\left(\hat{x}_{t}^{l}\right)=E\left(z_{t}^{\omega_{t}}\right), \quad \pi_{t}^{\omega_{t}}=\pi_{t}^{\omega_{t}}\left(\hat{x}_{t}^{l}\right) .
$$

Lower $\left(\mathrm{LB}^{L}\right)$ and upper $\left(\mathrm{UB}^{L}\right)$ bounds to the problem

$$
\mathrm{LB}^{L}=z_{M}^{L}, \quad \mathrm{UB}^{L}=\min \left\{\mathrm{UB}^{L-1}, \sum_{t=1}^{T}\left(c_{t} \hat{x}_{t}^{l}+z_{t}\left(\hat{x}_{t}^{l}\right)\right)\right\}, \mathrm{UB}^{0}=\infty .
$$

The solution procedure works analogously to the regular two-stage case. $\hat{x}_{t}^{l}$ is the optimal solution of the master problem in iteration $l$, and $\pi_{t}^{\omega_{t}}\left(\hat{x}_{t}^{l}\right)$ is the optimal dual solution of subproblem $\omega_{t}$, given $\hat{x}_{t}^{l} \cdot \alpha_{t}^{l}=0$ corresponds to feasibility cuts and $\alpha_{t}^{l}=1$ to optimality cuts. Solving the master problem in iteration $l$ we obtain a trial solution $\hat{x}_{t}^{l}$, which we pass to the subproblems. By solving a sample of subproblems $\omega_{t}, \omega_{t} \in N_{t}, t=1, \ldots, T$, according to the importance sampling scheme we compute estimates of the second-stage costs $z_{t}$ and estimates of the gradients $G_{t}^{l}$ and the righthand sides $g_{t}^{l}$ of the cuts. Note that there is one cut for each period $t$. The cuts are added to the master problem and the master problem is solved again. As before, the objective function value of the master problem gives a lower bound estimate, and the total expected costs of a trial solution $\hat{x}_{t}^{l}, t=1, \ldots, T$, gives an upper bound estimate of the objective function value of the problem. If the lower and the upper bound are sufficiently close according to a student $t$-test, the problem is considered to be solved. A $95 \%$ confidence interval of the optimal solution is then computed. 


\subsection{NUMERICAL RESULTS}

The method we have described above has been implemented. The FORTRAN code for solving general large-scale two-stage stochastic linear problems with recourse using Benders decomposition and importance sampling uses MINOS (Murtagh and Saunders (1983) [99]), which has been adapted for this purpose, as a subroutine for solving the linear programs of the master problem and the subproblems. Alternatively the code can also use a modified version of Tomlin's (1973) [120] LPM1 code of the revised simplex method as a subroutine. Versions of the code are installed on several computers, including the IBM 3090 mainframe, workstations such as Digital Equipment's DECstation 5000 and IBM's RS/6000, and on different personal computers.

All following test results are computed on a Toshiba T5200 laptop personal computer. First we present an illustrative example, a toy problem of expansion planning of power systems, which we discuss in detail. Then we derive numerical results from small test problems found in the literature. These problems are small enough that we are able to solve the universe case. Finally we demonstrate the solution of large-scale test problems with numerous stochastic parameters.

\subsubsection{Illustrative Example}

The illustrative example, test problem APL1P, is a model of a simple power network with one demand region. There are two generators with different investment and operating costs, and the demand is given by a load duration curve with three load levels: base, medium and peak. We index the generators with $j=1,2$, and the demands with $i=1,2,3$. The variables $x_{j}, j=1,2$, denote the capacities that can be built and operated to meet demands $d_{i}, i=1,2,3$. The variable $y_{i j}$ denotes the operating level for generator $j$ in load level $i$ with operating cost $f_{i j}$. The variable $y_{i s}$ defines the unserved demand in load level $i$ that can be purchased with penalty coist $f_{i s}>f_{i j}$. The subscript $s$ is not an index, but denotes only an unserved demand variable. The per-unit cost to build generator $j$ is $c_{j}$. Finally, the model is formulated with complete recourse, which means that for any given choice of $x$, demand is satisfied for all outcomes.

In this model, building new generators competes with purchasing unserved demand through the cost function, yet there is a minimum capacity $b_{i}$ that has to be built for each load level. The availabilities of the two generators, $\beta_{j}, j=1,2$, and the demands in each load level, $d_{i}, i=1,2,3$, are uncertain. Generator one has four possibilities, while generator two has five, and each demand has four. All of the data 
values are given in Table 2.1 and the problem can be formulated as follows:

$$
\begin{array}{rlrl}
\min \sum_{j=1}^{2} c_{j} x_{j}+E\left\{\sum_{j=1}^{2} \sum_{i=1}^{3} f_{i j} y_{i j}^{\omega}+\sum_{i=1}^{3} f_{i s} y_{i s}^{\omega}\right\} & \\
\mathrm{s} / \mathrm{t} & x_{j} & \geq b_{j} \quad j=1,2 \\
-\alpha_{j}^{\omega} x_{j}+\quad & \leq 0, \quad j=1,2 \\
& \sum_{i=1}^{3} y_{i j}^{\omega} & y_{i s}^{\omega} & \geq d_{i}^{\omega}, \quad i=1,2,3 \\
& \sum_{j=1}^{2} y_{i j}^{\omega}+\quad y_{i s} & \geq 0, \quad j=1,2, \\
x_{j}, & & & i=1,2,3 .
\end{array}
$$

Table 2.1: Model APL1P, test problem data

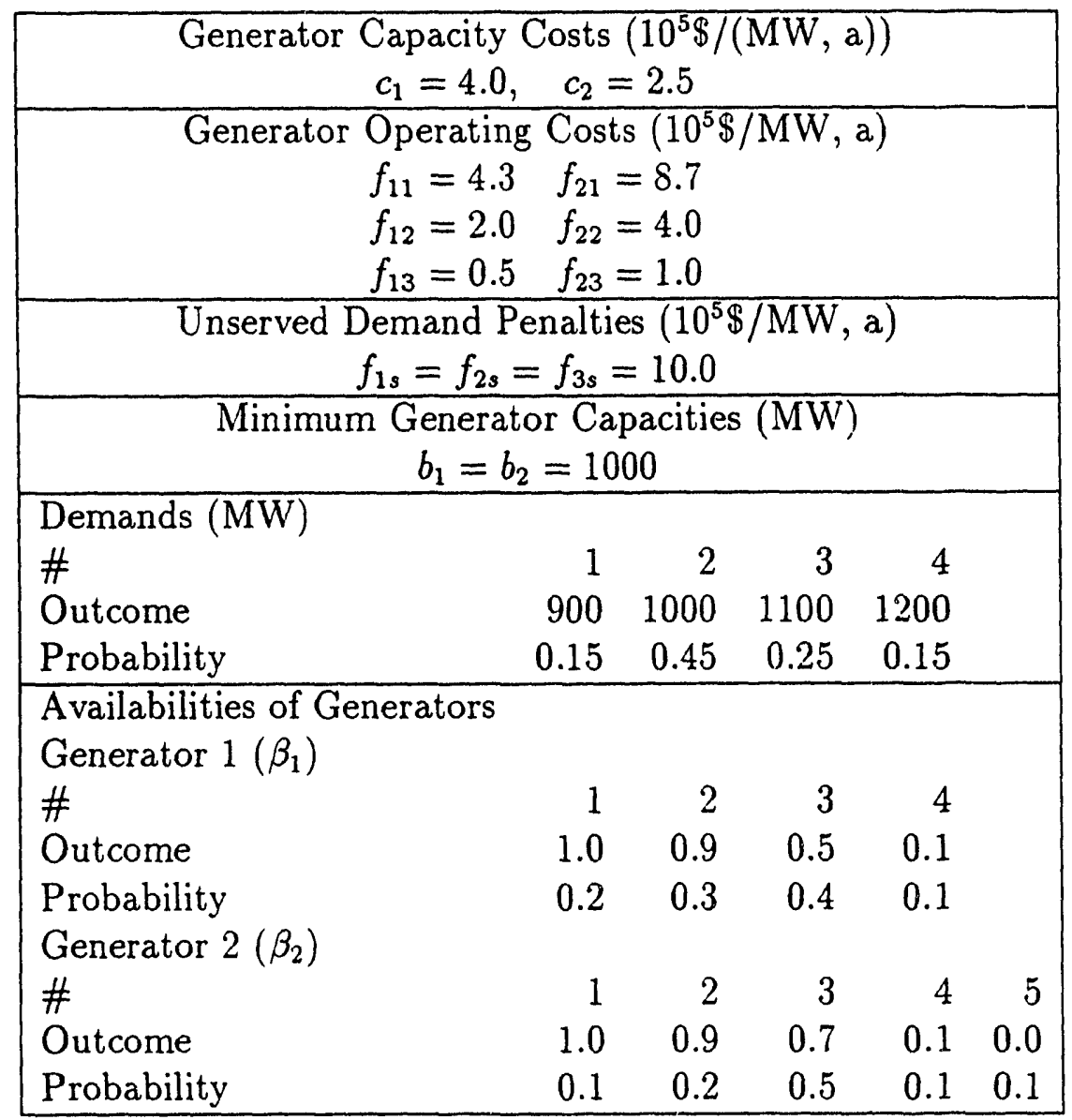

We will take $\omega \in \Omega$ when solving the universe problem and $\omega \in S$ when solving a problem with sampling.

The number of possible demands and availabilities results in $4 * 5 * 4^{3}=1280$ possible outcomes in $\Omega$, and thus 1280 subproblems have to be solved in each iteration 
of Benders decomposition for the universe case. We compare the universe solution with solutions gained by the importance sampling algorithm. Table 2.2 shows the results in the case of 20 samples out of the possible 1280 combinations and without an improvement phase. $\quad 100$ replications of the same experiment with different

Table 2.2: Model APL1P, 20 samples (100 replications of the experiment)

\begin{tabular}{|lrrrr|}
\hline & correct & mean & $\begin{array}{r}95 \% \text { conf } \\
\%\end{array}$ & $\begin{array}{r}\text { bias } \\
\%\end{array}$ \\
\hline $\begin{array}{l}\text { \#univ } \\
\text { \#iter }\end{array}$ & 1280 & & & \\
\hline G1 & 1800.0 & 1666.5 & 57.0 & -7.4 \\
G2 & 1571.4 & 1732.5 & 52.5 & 10.2 \\
theta & 13513.7 & 13729.4 & 21.3 & 1.6 \\
\hline obj & 24642.3 & 24726.7 & 2.1 & 0.3 \\
\hline est. conf (\%) & left & 1.5 & & \\
est. conf (\%) & right & 1.9 & & \\
coverage & & 0.90 & & \\
\hline
\end{tabular}

seeds were run to get statistical information about the accuracy of the solution and the estimated confidence interval. The mean over the 100 replications of the objective function value (total costs) differs from the universe solution by $0.3 \%$. From the distribution of the optimum objective value derived from the 100 replications of the experiment a $95 \%$ confidence interval is computed: $\pm 2.1 \%$. In each replication a $95 \%$ confidence interval of the solution is estimated. The mean over all replications of the estimated confidence interval is $1.5 \%$ on the left side and $1.9 \%$ on the right side. In the worst case an objective function value of 26233.9 was computed. This is about $6.4 \%$ off the correct answer. The estimated $95 \%$ confidence interval in this case did not cover the correct answer. The coverage rate of $90 \%$ expresses that in $90 \%$ of the 100 replications the correct answer of the universe solution is covered by the estimated confidence interval. This shows that if using a sample size of 20 we are slightly underestimating the confidence interval: if the computation of the $95 \%$ confidence interval were exact, we would expect a coverage rate of $95 \%$. The reason for the underestimation of the $95 \%$ confidence interval in the case of sample size 20 lies in the underlying assumptions of the estimation method, e.g. a normal error distribution for a sample sizes of 20 , constant error distribution along a cut, and the same basis for all outcomes of the random right hand sides of the cuts. Especially the latter assumption is only true, if the variances are small. A larger sample size reduces the variances and we expect a better coverage rate of the $95 \%$ confidence interval. The bias and the confidence interval of the optimum strategies (the loads $x$ to be installed) are larger than those of the optimum objective function 
value. The objective function near the optimal solution appears to be flat: several different strategies lead close to the optimum costs. Confidence intervals for the two components of $x$ of about $57 \%$ and $52 \%$ are computed. In the above example a sample size of 20 was chosen. Note that additional computational effort is also needed to obtain the importance distribution, e.g. 17 subproblems have to be solved in each iteration to obtain the marginal costs $M_{i}$. Compared to the universe solution the method achieves with about $2.9 \%$ the computational effort a solution that is with $95 \%$ confidence within an interval of $\pm 2.1 \%$ of the correct answer. We conclude that importance sampling seems to be a promising approach to solving stochastic linear programs.

Table 2.3 represents the results when using 200 samples. One can see decreasing bias, decreasing confidence intervals and improving estimations of the confidence intervals with increased sample size. The coverage of the $95 \%$ confidence interval, computed by 100 replications of the experiment with different seeds, is now $95 \%$.

Table 2.3: Model APL1P, 200 samples (100 replications of the experiment)

\begin{tabular}{|lrrrr|}
\hline & correct & $\begin{array}{r}\text { mean } \\
\text { \# }\end{array}$ & $\begin{array}{r}95 \% \text { conf } \\
\%\end{array}$ & $\begin{array}{r}\text { bias } \\
\%\end{array}$ \\
\hline $\begin{array}{l}\text { \#univ } \\
\text { \#iter }\end{array}$ & 1280 & & & \\
\hline G1 & 1800.0 & 1728.7 & 31.5 & -4.0 \\
G2 & 1571.4 & 1681.7 & 29.2 & 7.0 \\
theta & 13513.7 & 13554.7 & 12.2 & 0.3 \\
\hline obj & 24642.3 & 24673.8 & 0.4 & 0.1 \\
\hline $\begin{array}{l}\text { est. conf (\%) } \\
\text { est. conf (\%) }\end{array}$ & left & 0.4 & & \\
coverage & right & 0.7 & & \\
\hline
\end{tabular}

\subsubsection{Test Problems from the Literature}

We investigate the performance of the algorithm on two other examples from the literature, which are small enough to compute the universe solution. PGP2, derived from Louveaux and Smeers (1988) [88], is a power generation planning model used to determine the capacities of various types of equipment required to ensure that consumer demand is met. The demands in 3 demand regions are stochastic and represented by discrete random variables with 9,9 and 8 outcomes. CEP1 is a capacity planning model for a manufacturing plant in which several parts are produced on several machines. If the demand for the parts exceeds the production capability the residual parts are purchased from external sources at a price much higher than the 
production costs to meet the demand. There are 3 stochastic parameters (demands for parts), with discrete and uniform distribution with 10 outcomes each. The formulations and data for CEP1 and PGP2 may be found in Higle, Sen and Yakowitz (1990) [63].

In the case of PGP2 we obtained very accurate results using a sample size of 50 . By computing 100 replications of the experiment we find that the mean of the objective function values differs $0.1 \%$ from the correct answer. The $95 \%$ confidence interval of the objective function value, computed by the 100 replications of the experiment, is $\pm 0.76 \%$. The mean of the confidence intervals estimated in each replication is $0.62 \%$ on the left side and $0.9 \%$ on the right side. In $98 \%$ of the cases the correct solution is covered by the $95 \%$ confidence interval. In the worst case the solution differed by $0.77 \%$ from the correct answer and was not covered by the $95 \%$ confidence interval.

In the case of CEP1 a higher sample size is needed to obtain accurate results. The estimation of the second-stage costs appears to be more difficult. The reason lies in the fact that the (penalty) costs of buying parts from external sources are much higher than the costs of production. For this problem the additive approximation function is not a very good approximation to the true cost function, as it does not cover the very high costs in scenarios where all 3 demands are high. The estimated confidence interval seems to be large. We computed $4.65 \%$ on the left side and $4.62 \%$ on the right side (mean over 100 replications of the experiment). The estimations of the confidence interval are accurate as indicated by the coverage rate of $95 \%$ of the correct answer by the $95 \%$ confidence interval. In the worst case a difference of $8.07 \%$ of the objective function value to the correct answer was computed. The worst case solution is not covered by the estimated confidence interval. In this example it is easier to compute the value of the first stage variables than to estimate the secondstage costs. In most cases the correct answer of the first stage variables was obtained. We have developed methods that adaptively improve the approximation function if sample information shows that the variance of the estimation is too large. Table 2.4 and Table 2.5 represent the computational results of PGP2 and CEP1 and show the size of the test problems.

\subsubsection{Large-Scale Test Problems}

In the following we report on the solution of large-scale test problems with several stochastic parameters. These problems are so large that it is impossible to compute the universe solution.

\section{Facility Expansion Planning}

WRPM is a prototype multi-area capacity expansion planning problem for the western USA and Canada. The model is detailed, covering 6 regions, 3 demand blocks, 2 seasons, and several kinds of generation and transmission technologies. The objective is to determine optimum discounted least-cost levels of generation and transmission 
Table 2.4: Model PGP2, 50 samples (100 replications of the experiment)

\begin{tabular}{|lrrrr|}
\hline & correct & mean & $\begin{array}{r}95 \% \text { conf } \\
\%\end{array}$ & $\begin{array}{r}\text { bias } \\
\%\end{array}$ \\
\hline \#univ & & & & \\
\#iter & 648 & & & \\
\hline obj & 392.2 & 392.5 & 0.76 & 0.1 \\
\hline est. conf (\%) & left & 0.62 & & \\
est. conf (\%) & right & 0.9 & & \\
coverage & & 0.98 & & \\
comp. time (min) & & 0.28 & & \\
\hline Problem Size & & & & \\
Master: & rows & 3 & & \\
& columns & 7 & & \\
Sub: & nonzeros & 16 & & \\
& rows & 8 & & \\
& columns & 16 & & \\
\hline
\end{tabular}

facilities for each region of the system over time. The model minimizes the total discounted costs of supplying electricity (investment and operating costs) to meet the exogenously given demand subject to expansion and operating constraints. A description of the model can be found in Dantzig et al. (1989) [25] and Avriel, Dantzig and Glynn (1989) [3]. In the stochastic version of the model the availabilities of generators and transmission lines and demands are subject to uncertainty. There are 13 stochastic parameters per time period ( 8 stochastic availabilities of generators and transmission lines and 5 uncertain demands) with discrete distributions with 3 or 4 outcomes. The operating subproblems in each period are stochastically independent. WRPM has been developed in three flavors. The test problem WRPM1 covers a time horizon of 1 future period, and WRPM2 covers 2 future periods of 10 years each. WRPM1 and WRPM2 are reduced versions of WRPM3, the largest problem with the most realistic formulation. There are differences in the parameters between WRPM1, WRPM2 and WRPM3. Note that in the deterministic equivalent formulation the problem would have more than 1.5 billion (WRPM1) and more than 3 billion (WRPM2) equations.

Computational results for these large-scale test problems are represented in Table 2.6. Besides the solution of the stochastic problems, Table 2.6 shows the results from solving the expected value problem. In this case the stochastic parameters are replaced by their expectations to obtain a deterministic problem. The expected value solution is then used as a starting point for the stochastic solution. We also report 
Table 2.5: Model CEP1, 200 samples (100 replications of the experiment)

\begin{tabular}{|lrrrr|}
\hline & correct & mean & $\begin{array}{r}95 \% \text { conf } \\
\%\end{array}$ & $\begin{array}{r}\text { bias } \\
\%\end{array}$ \\
\hline \#univ & 1000 & & & \\
\#iter & & 6.4 & & \\
\hline obj & 57790.7 & 58832.7 & 4.63 & 1.8 \\
\hline est. conf (\%) & left & 4.65 & & \\
est. conf (\%) & right & 4.62 & & \\
coverage & & 0.95 & & \\
comp. time (min) & & 0.28 & & \\
\hline Problem Size & & & & \\
Master: & rows & 12 & & \\
& columns & 10 & & \\
Sub: & nonzeros & 36 & & \\
& rows & 9 & & \\
& columns & 16 & & \\
\hline
\end{tabular}

on the estimated expected costs of the expected value solution. These are the total expected costs that would occur if the xpected value solution were implemented in a stochastic environment. The objective value for the true stochastic solution must lie between the objective value of the expected value solution and the expected costs of the expected value solution.

In all cases (WRPM1, WRPM2 and WRPM3) we chose a sample size of 100 . The estimate of the objective function value of the stochastic solution (289644.2 in case of WRPM1 and 143109.2 in case of WRPM2) is amazingly accurate. The $95 \%$ confidence interval was computed as $0.0913 \%$ on the left side and $0.063 \%$ on the right side (WRPM1) and $0.0962 \%$ on the left side and $0.1212 \%$ on the right side (WRPM2). Thus the objective value of the stochastic solution lies with $95 \%$ probability within $289379.7 \leq z^{*} \leq 289826.0$ (WRPM1) and $142971.5 \leq z^{*} \leq 143282.6$ (WRPM2). In both cases the expected costs of the expected value solution and the expected costs of the stochastic solution differ significantly. The solution time on a Toshiba T5200 laptop PC with 80387 mathematic coprocessor was 75 minutes (WRPM1) and 187 minutes (WRPM2). During this time about 7500 (WRPM1) and 15700 (WRPM2) subproblems (linear programs of the size of 302 rows and 289 columns) were solved.

The realistic model WRPM3 covers a time horizon of 3 future periods of 10 years each. Thus the total number of stochastic parameters is 39 . The number of universe scenarios is larger than $5 \cdot 10^{6}$ per period. In the deterministic equivalent formulation the problem, if it were possible to state it, would have more than 4.5 billion 
Table 2.6: Large test problems: computational results for power planning

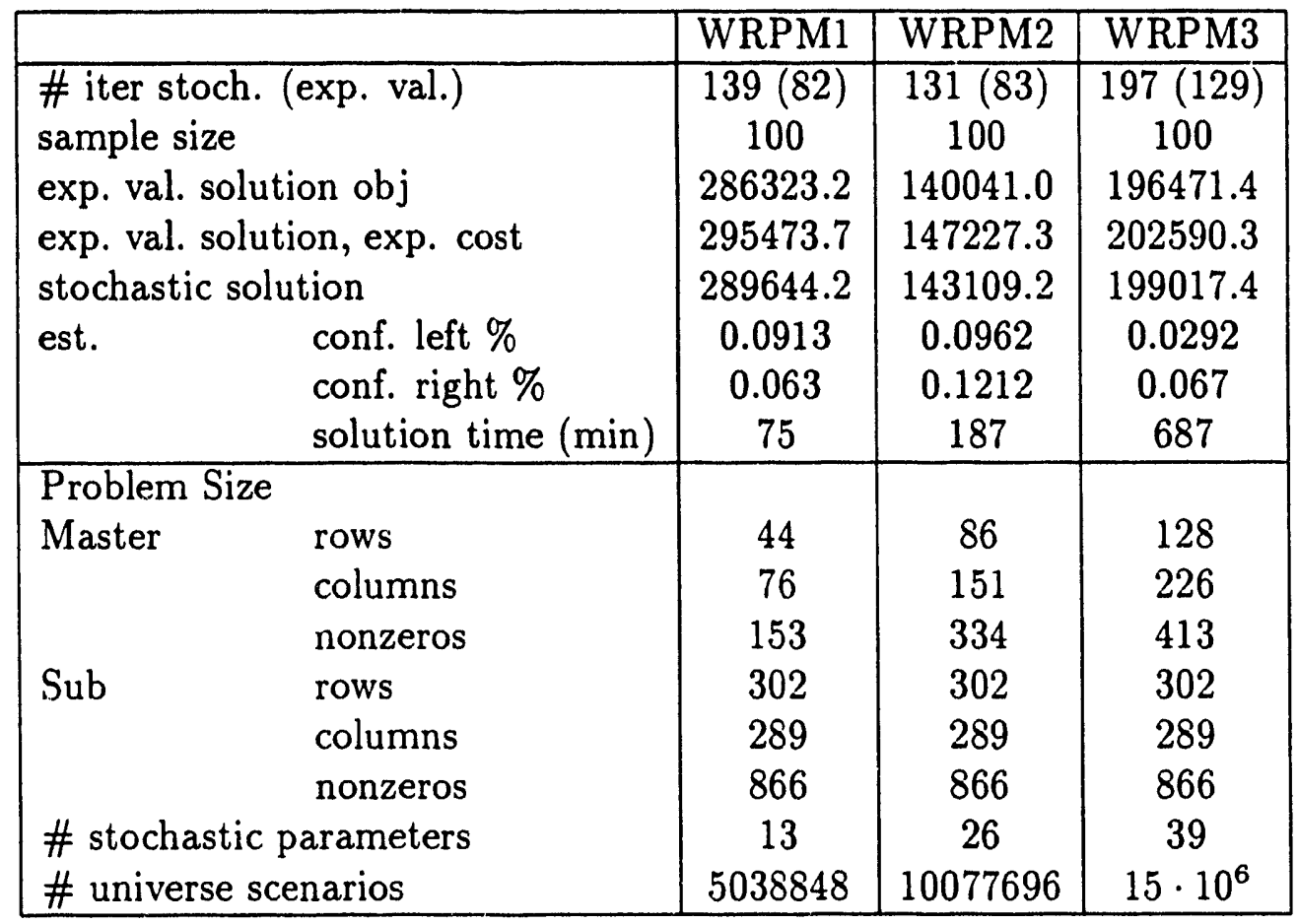

constraints.

The stochastic WRPM3 was solved by using a sample size of 100 per period. It took 129 iterations to obtain the expected value solution and an additional 68 iterations to compute the stochastic solution. The objective function value of the stochastic solution was estimated as 199017.4 with an remarkably small $95 \%$ confidence interval of $0.029 \%$ on the left side and $0.067 \%$ on the right side. Thus the optimal solution lies with $95 \%$ confidence between $198959.3 \leq z^{*} \leq 199164.1$. The expected costs of the expected value solution (202590.3) and the objective function value of the stochastic solution differ significantly from the expected costs of the optimal stochastic solution. The problem was solved in 687 minutes on the Toshiba T5200. This includes time to solve 26295 linear subproblems with 302 rows and 289 columns, and 197 master problems.

Note that the solution time of WRPM1, WRPM2 and WRPM3 does not increase linearly with the size of the system. WRPM3 takes significantly longer to be solved than WRPM1 and WRPM2. We have used LPM1 to solve WRPM1 and WRPM2 and MINOS for solving WRPM3. While the LPM1 code is less sophisticated and less reliable than MINOS, it solves a series of subproblems, where we start each optimization from the optimal solution of the previous subproblem, faster than MINOS. This is primarily because LPM1 has less computational overhead than MINOS. In 
addition to the difference in the code used for solving the problems, WRPM3 takes more iterations than WRPM1 and WRPM2, which results in more computational work to be performed and in a larger solution time.

\section{Portfolio Management}

Computational results from portfolio management problems can be found in Table 2.7. FI12 is an example, formulated as a network problem. It is a modified version

Table 2.7: Large test problems: computational results for financial planning

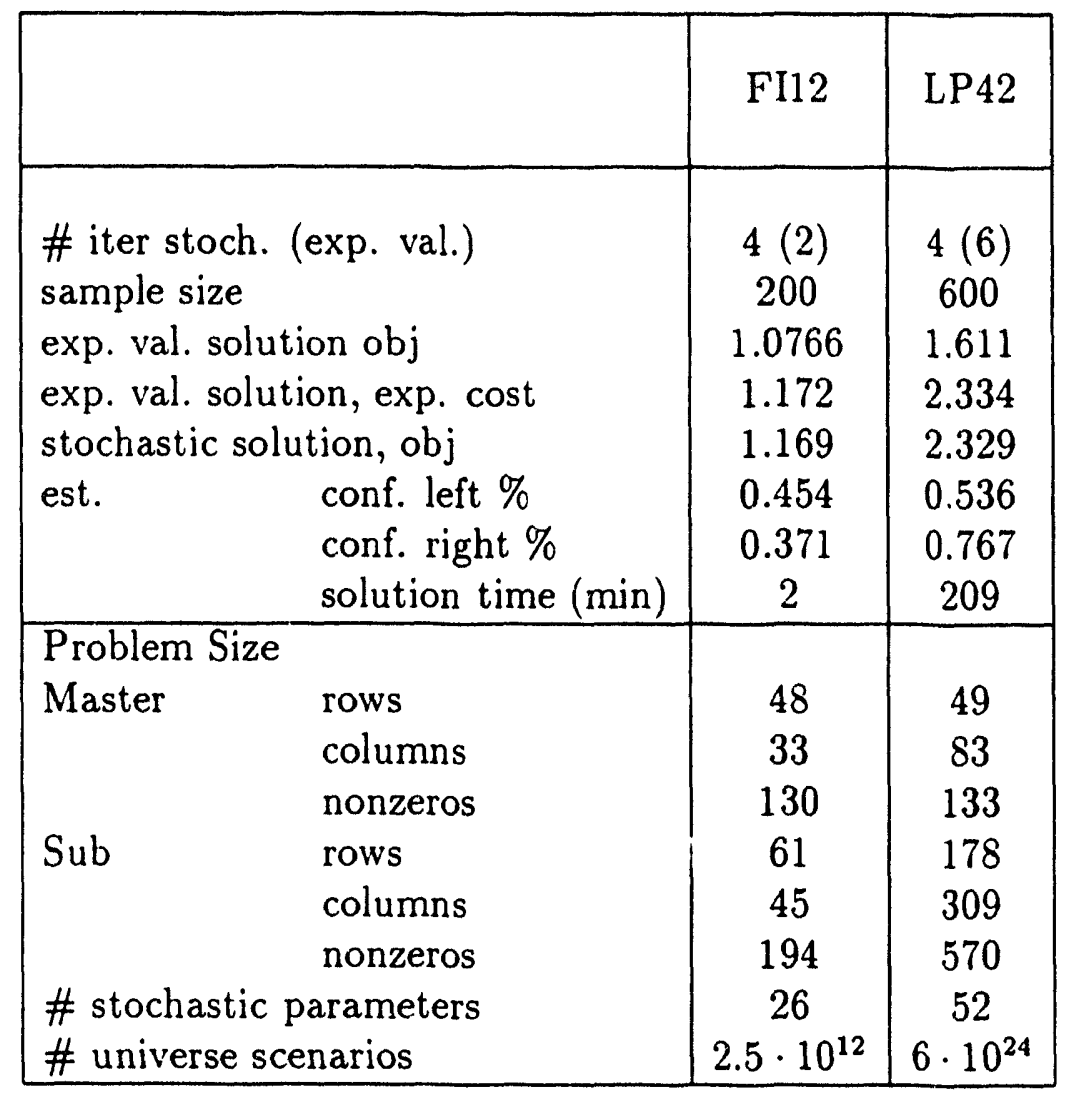

of test problems found in Mulvey and Vladimirou (1989) [96]. The problem is to select a portfolio that maximizes expected returns in future periods taking into account the possibility of revising the portfolio in each period. There are also transaction costs and bounds on the holdings and turnovers. The test problem FI12 covers a planning horizon of two future periods. The returns of the stocks in the two future periods are stochastic parameters. The problem is formulated as a two-stage problem. Rather than solving the problem by looking at a certain number of preselected scenarios (18 to 72 in the case of Mulvey and Vladimirou) we instead assumed the returns of the stocks in the future periods to be independent random parameters, discretely 
distributed with 3 outcomes each. As there are 13 stocks with uncertain returns, the problem has 26 stochastic parameters. The universe number of scenarios $\left(2.5 \cdot 10^{12}\right)$ is very large, so that the deterministic equivalent formulation of the problem has more than $10^{14}$ rows. The stochastic parameters appear in the $B$ matrix as well as in the $D$ matrix.

A sample size of 200 was chosen for solving test problem FI12. The problem was solved in only 4 iterations. The objective function value of the stochastic solution is computed as 1.1695 with a $95 \%$ confidence interval of $0.454 \%$ on the left side and $0.371 \%$ on the right side. Thus with $95 \%$ probability the optimal solution lies in the range $1.164 \leq z^{*} \leq 1.174$. The estimated expected costs of the expected value solution (1.172) lie within the $95 \%$ confidence interval of the costs of the stochastic solution. However, in this case the expected costs of the expected value solution and the expected costs of the stochastic solution differ significantly.

LP42 is also a portfolio management test problem, formulated as a network problem. It is a modified version of test problems found in Mulvey and Vladimirou (1989) [96] and of the same structure as FI12 but much larger. Again, there are transaction costs and bounds on the holdings and turnovers. The test problem covers a planning horizon of four future periods. The returns of the stocks in the four future periods are assumed to be independent stochastic parameters, discretely distributed with 3 outcomes each; this formulation differs from that of Mulvey and Vladimirou who restricted the problem size by looking at a certain number of preselected scenarios. As in Mulvey and Vladimirou the multi-period problem is viewed as a two-stage problem, where all future periods are included in the second stage. With 13 stocks with uncertain returns, the problem has 52 stochastic parameters. The universe number of scenarios $6 \cdot 10^{24}$ is very large, so that the deterministic equivalent formulation of the problem if expressed explicitly would have more than $1.9 \times 10^{27}$ rows. Here, the stochastic parameters appear in the $B$ matrix as well as in the $D$ matrix. Due to the stochasticity of the $D$ matrix cuts from the expected value problem are not valid for the stochastic problem. The expected value problem and the stochastic problem were solved separately. A sample size of 600 was chosen, and the solution (objective function value 2.329) was obtained in 4 iterations. The $95 \%$ confidence interval is very small given the large number of stochastic parameters, namely $0.536 \%$ on the left side and $0.767 \%$ on the right side. Thus with $95 \%$ confidence the objective value of the optimal solution lies within $2.316 \leq z^{*} \leq 2.347$. The expected costs of the expected value solution are significantly different from the expected costs of the stochastic solution. 


\section{Chapter 3}

March 31, 1993

\section{Using Parallel Processors}

In collaboration with James $\mathrm{K}$. Ho we have explored how our approach for solving two-stage stochastic linear programs can be effectively implemented on a parallel (Hypercube) multicomputer (Dantzig, Ho and Infanger (19y1) [26]).

\subsection{HYPERCUBE MULTICOMPUTERS}

Advances in VLSI (very large-scale integration) for digital circuit design are leading to much less expensive and much smaller computers. They have also made it possible to build a variety of "supercomputers" consisting of many small computers combined into an array of concurrent processors. We shall refer to such an architecture as multicomputers. Each individual processor is called a node. Typically, the nodes are the same kind as those used in high-end microcomputers and are relatively inexpensive. Significant computational power can be obtained by making many of them work in parallel at costs that are much lower than an equivalent single processor. Obviously, the effectiveness of the approach depends on whether an application can be reduced to a well-balanced distribution of asynchronous tasks on the nodes. Linear programming and especially stochastic linear programs solved by decomposition naturally fit into this framework.

A Hypercube multicomputer is essentially a network of $2^{n}$ processors interconnected in a binary $n$-cube (or hypercube) topology. The connections for $n \leq 4$ are illustrated in Figure 3.1. Each processor (or node) has its local memory and runs asynchronously of the others. Communication is done by means of messages. A node can communicate directly with its $n$ neighbors. Messages to more distant nodes are routed through intermediate nodes. The hypercube topolrgy provides an efficient balance between the costs of connection and the benefits of direct linkages. Usually, a host computer serves as an administrative console and as a gateway to the hypercube for users.

For the work reported here, we used an Intel $\mathrm{PSC} / 2 \mathrm{~d} 6$ with 64 nodes at the Oak Ridge National Laboratory. Each node consists of Intel's 32-bit 80386 CPU (4 MIPS) coupled with an 80387 (300 Kflops) numeric coprocessor for floating-point acceleration. It has 4 MBytes of local memory. The hypercube (or Cube) is accessed via a host (or System Resource Manager) which is also a 80386-based system with 8 MBytes of memory and a 140 MByte hard disk. The operating system on the host 

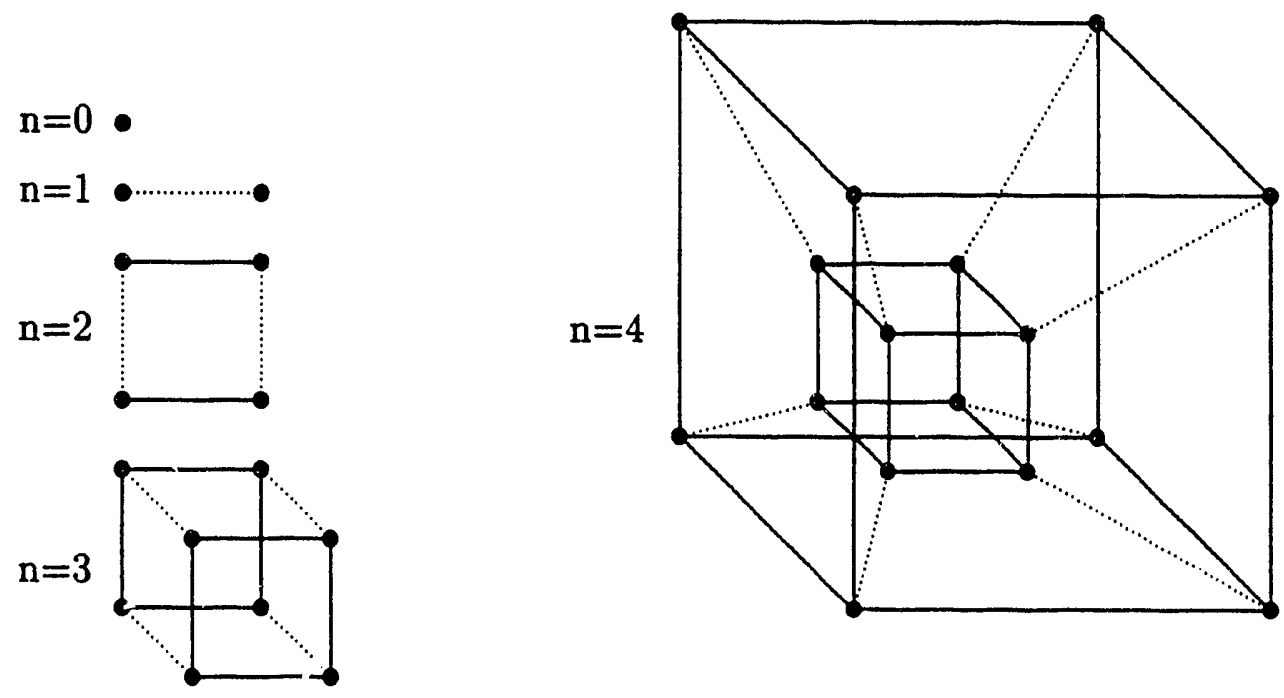

Figure 3.1: Hypercube multicomputers of dimensions $n \leq 4$

is the Unix System V/386 (Release 3.0). The data transfer rate between the System Resource Manager and the Cube has a peak value of $2800 \mathrm{KBytes} / \mathrm{sec}$.

Altho..gh the nodes are physically connected as the edges of a hypercube, a trade-marked routing network called DIRECT-CONNECT provides essentially uniform communication linkages between all the nodes. Therefore the hypercube can be programmed as an ensemble of processors with an arbitrary communications network in which each node can communicate more or less uniformly with all other nodes. The host machine allows the user to perform the following tasks:

- To edit, compile and link host/r.ode programs.

- To access and release the cube (or a partition thereof).

- To execute the host program.

- To start or kill processes on the cube.

Operations peculiar to the hypercube are controlled either by Unix-type commands (iPSC/ 2 commands) or by extensions to standard programming languages such as Fortran and C (iPSC $/ 2$ routines). The iPSC $/ 2$ commands and routines for the Fortran 
programming environment are documented in Intel (1988) [74] and [75]. To execute a typical parallel program, the following steps are used.

- Compile and link the host and node programs to create executable modules.

- Obtain a partition of the cube (a sub-cube) of suitable size. For example, a call for a sub-cube of dimension 3 allocates to the user an exclusive sub-cube of 8 processors identified by the node numbers $0,1,2, \ldots, 7$.

- Run the host program. Node programs are loaded on to the appropriate nodes at runtime in response to calls in thc host program.

- On termination, kill all node processes and flush messages.

- Relinquish access to the sub-cube.

Internodal and host-to-node communication is done by subroutine calls in the corresponding programs.

\subsection{THE PARALLEL ALGORITHM}

The Hypercube computer has the architeciure of loosely coupled multiprocessors. The nodes of the cube are independert processors, where each processor has its own operating system and its own memory. The nodes are connected via a communication network. Information is exchanged between nodes only by sending messages. The hyperc"be architecture defines which nodes are directly connected and which nodes are only indirectly connected via third nodes. Message routing systems of modern Hypercube computers, like the Intel iPSC/2 computer that we are using, ensure that communication between indirectly connected nodes is very fast. Thus the difference in the communication time between directly and indirectly connected nodes is negligible. However, the time spent for communication can be significant if much information is exchanged between nodes. Therefore the design of a parallel algorithm for loosely connected multiprocessors should be laid out in such a way that only minimum amounts of information have to be exchanged between nodes.

The main work is in the repeated solving of the master problem, and also the solving of the subproblems in the preparatory phase and in the sample phase. All other tasks are comparatively unimportant with respect to computing time. We assign processor 0 to be the master processor. Besides its main task of solving the master problem, the master processor also controls the computation and synchronizes the algorithm. The other processors (1-63) were assigned to be sub processors, with the main task of solving subproblems. This design requires communication between the master processor and the sub processors. No information needs to be exchanged between different sub processors.

In addition there is a host processor that has access to data storage devices and manages data input and output. The execution of the parallel program follows the 
following general steps. The host processor loads the host module (the executable file for the host processor) into its memory and starts the execution. Next the executable files for the master processor and the sub processors are loaded into the host and then sent to the master processor and the sub processors respectively. The master processor and the sub processors after they receive their modules start execution. After processing the input data and sending it to master and subs, the host remains inactive and waits until it receives the optimal solution from the master processor. During this time the algorithm is performed entirely in the cube and the master processor controls the execution of the program. After receiving the optimal solution, the host processor outputs the solution to the disk, stops the execution of the programs of the master and sub processors, and releases the cube, terminating the parallel program.

The problem data includes the problem specification of the master and the sub, the stochastic information and control parameters for the execution of the program. The input data for specifying the master problem and the subproblem are given in the form of an MPS file. Internally the problems are stored in the form of the data structures used by the linear programming solver, which we use as a subroutine. We adapted LPM1 (Tomlin 1973) [120], a linear programming optimizer, for our purposes. Clearly, the master processor only receives the data for the master problem and the sub processors only get the data for the subproblem. Thus no switching between different problems is necessary, as it would be in a serial implementation. Both master and sub processor receive the complete stochastic information. The stochastic data include the identification of the stochastic parameters within the problem and their distributions.

An index vector $\nu^{\omega}=\left(\nu_{1}, \ldots, \nu_{h}\right)^{\omega}$ completely defines a scenario $\omega$. We define $\nu_{i} \in \Omega_{i}$ or $\nu_{i}=1, \ldots, k_{i}, i=1, \ldots, h$. For example $\nu^{\omega}=(1,3,2)$ would denote a scenario given by the first outcome of random parameter 1 , the third outcome of random parameter 2 and the second outcome of random parameter 3 . Thus only the index vector $\nu^{\omega}$ is transmitted from the master processor to a sub processor to identify the scenario subproblem to be solved. For example, for $h=20$ and a 4 -byte integer representation, 80 bytes have to be sent. Besides the scenario information $\nu^{\omega}$ the current solution of the master problem $\hat{x}^{l}$ is needed to set up the scenario problem $\omega$. We only pass $\hat{x}^{l}$ to each sub processor $j$ once per iteration at the beginning of the preparation phase. The flag $I_{x} \in\{0,1\}$ tells the sub processor if an $\hat{x}$ has to be received (1) or not $(0)$.

Now sub processor $j$ looks up the outcomes of the stochastic parameters corresponding to $\nu$ to set up the vector $b(\nu)$ and the matrix $B(\nu)$. Using $\hat{x}$ the right hand side $b(\nu)+B(\nu) \hat{x}$ is computed and the subproblem is solved.

In all cases the optimal objective function value $z(\nu)$ has to be sent to the master processor. Dual information for the coefficients $G$ and the right-hand side $g$ of the cut is all that is needed from the base-case scenario and all sample scenarios. In this case we compute the products $G(\nu)=B(\nu) \pi(\nu)$ and $g(\nu)=b(\nu) \pi(\nu)$ and send the result to the master processor. The flag $I_{c}$ tells the sub processor if the computation 
and the sending of $G(\nu)$ and $g(\nu)$ is requested (1) or not (0).

In our design the sub processors do not have any information of the status of the algorithm. The sub processors set up and solve the subproblems and post-process the solution. The computation is controlled by the master processor through the flags $I_{x}$ and $I_{c}$.

The master processor runs the entire algorithm except for obtaining solutions of subproblems. An important task concerns the controlling of the assignment of subproblems $\omega$ to sub processors $j$ in the case where more sample subproblems have to be solved per iteration than there are sub processors available. Assigning subproblems in equal proportions to sub processors is not always possible for all sample sizes, nor is it most efficient. Different subproblems need different amounts of time to be solved. The solution time mainly depends on how many columns of the starting basis (from which the solving procedure is started) differs from the optimal basis of the subproblem. Clearly, it makes sense and is convenient to use as starting basis the optimal basis of the subproblem that was last solved on the same processor.

We implemented an algorithm to adaptively balance the work load of the sub processors. In our scheme the master processor keeps track of whether a sub processor $j$ is busy or idle. At the beginning of each solving phase (preparatory and sample phase) all sub processors are idle. The master processor starts a sub processor working by sending the first message $\left(I_{x}, I_{c}\right)$ to it. At this time the sub processor is set to busy. It is set to idle again when its solution has arrived at the master processor. Given a queue of subproblems to be solved, the first subproblem in the queue is assigned to the next idle sub processor. The master processor keeps switching between sending out problems and receiving solutions until all subproblems are solved. Of course the mapping $\omega \rightarrow j$ is not unique because different subproblems $\omega$ are solved by one sub processor $j$. However, because we only send a new problem after the solution of the previous problem has been received, the solution of a subproblem $\omega$ can be identified as uniquely coming from sub processor $j$.

We can now summarize and state the algorithm. Step 2 is computationally the most expensive part and is the part performed by using parallel processors.

\section{The Algorithm}

\section{Host processor}

Step H: 0.0 Load host executable module from disk.

Step H: 0.1 Load master module from disk.

Send master module to processor 0 .

Load sub module from disk

Send sub module to processors $j, j=1, \ldots, J$.

Step H: 0.2 Read data and from disk.

Send control data and stochastic data to processors $j, j=0, \ldots, J$. 
Send master problem data to processor 0 .

Send subproblem data to processors $j, j=1, \ldots, J$.

Step H: 6 Receive optimal solution.

Write solution report.

Kill cube. Stop.

\section{Master Processor}

Step M: 0 Receive master module from host processor.

Receive control and stochastic data from host processor.

Receive master problem data from host processor.

Initialize: $l=0, \mathrm{UB}^{0}=\infty$.

Step M: 1 Solve the relaxed master problem.

Obtain a trial solution $\hat{x}^{l}$ and a lower bound $\mathrm{LB}^{l}$.

Step M: $2.0 l=l+1$.

Step M: 2.1 Determine preparatory scenarios $\nu^{\omega}=\left(\nu_{1}, \ldots, \nu_{h}\right)^{\omega}, \omega=1, \ldots, n_{\text {prep }}$.

Step M: $2.2 \omega=1, \ldots, n_{\text {prep : }}$

Determine $\omega \longrightarrow j$.

Send $I_{x}^{j}, I_{c}^{\omega}$ to sub processor $j$.

Send $\hat{x}^{l}$ to sub processor $j$.

Send $\nu^{\omega}$ to sub processor $j$.

$\omega=1, \ldots, n_{\text {prep : }}$

Receive $z^{\omega}$ from sub processor $j$.

If $I_{c}^{\omega}=1$ : Receive $G^{\omega}, g^{\omega}$ from sub processor $j$.

Step M: 2.3 Compute the importance distribution.

Step M: 2.4 Sample scenarios $\nu^{\omega}=\left(\nu_{1}, \ldots, \nu_{h}\right)^{\omega}, \omega=1, \ldots, n$ from the importance distribution.

Step M: 2.5 For $\omega=1, \ldots, n$ :

Determine $\omega \longrightarrow j$.

Send $I_{x}^{j}, I_{c}^{\omega}$ to sub processor $j$.

Send $\nu^{\omega}$ to sub processor $j$.

For $\omega=1, \ldots, n$ :

Receive $z^{\omega}$ from sub processor $j$.

Receive $G^{\omega}, g^{\omega}$ from sub processor $j$.

Step M: 2.6 Obtain estimates of the expected second stage cost, the coefficients and the right-hand side of the cut. Add the cut to the master problem. Obtain an upper bound $\mathrm{UB}^{l}$. 
Step M: 3 Solve the master problem. Obtain a trial solution $\hat{x}^{l}$ and a lower bound $\mathrm{LB}^{l}$.

Step M: $4 s=\mathrm{UB}^{l}-\mathrm{LB}^{l}+T O L$ If $s \geq 0$ (student t-test) go to Step 2 .

Step M: 5 Obtain a solution and compute confidence interval.

Step M: 6 Send optimal solution to host processor.

\section{Sub Processor $j$ :}

Step S: 0 Receive sub-module from host processor.

Receive control and stochastic data from host processor.

Receive subproblem data from host processor.

Step S: 2.1 Receive $I_{x}, I_{c}$ from the master processor.

If $I_{x}=1$ : Receive $\hat{x}$ from the master processor.

Receive $\nu$ from the master processor.

Step S: 2.2 Compute $B(\nu), b(\nu)$ and the right-hand side $b(\nu)+B(\nu) \hat{x}$.

Step S: 2.3 Solve scenario subproblem $\nu$.

Step S: 2.4 Send $z(\nu)$ to the master processor.

Step S: 2.5 If $I_{c}=1$ :

Compute $G(\nu)=\pi(\nu) B(\nu), g(\nu)=\pi(\nu) b(\nu)$.

Send $G(\nu), g(\nu)$ to the master processor.

Step S: 2.6 Go to Step S: 2.1

\subsection{PERFORMANCE MEASURES}

The main purpose of parallel processing is to reduce computing time relative to conventional sequential computation. In the case when large sample sizes are necessary in order to obtain good approximate solutions to stochastic linear programs, parallel processing is an important part of the solution technique, because the solution times on sequential computers may be excessive.

Assuming that a number $p$ of processors are available and allocated to solve the problem at hand, we compare the parallel time utilizing $p$ processors to the sequential time using only one processor. We define the parallel time $t_{p}$ the duration from start to finish of the solution process in the parallel implementation. In terms of CPU times, $t_{p}$ covers the disjoint union (non-overlapping total) of CPU times of all processors. We define the sequential time $t_{s}$ to be the sum of all CPU times of all processors. The sequential time $t_{s}$ differs from a sequential time obtained by actually soiving the 
problem on one processor. This would require a different implementation and would not be directly comparable. In a serial version no messages are sent. On the other hand, computing resources are needed for alternately switching between solving the master problem and the subproblems.

The speedup $S$ in using $p$ processors instead of one is given by

$$
S=\frac{t_{s}}{t_{p}} .
$$

The efficiency is defined by

$$
E=\frac{S}{p} \times 100 \% .
$$

A simple set of algebraic formulae can be used to predict the sequential time $t_{s}$ and the parallel time $t_{p}$. We denote by $t_{\mathrm{MA}}$ the mean duration to compute the tasks assigned to the master processor per iteration. We define $t_{\mathrm{SUB}}$ to be the mean duration to compute the tasks assigned to a sub processor (mainly solving one subproblem) when starting from the optimal solution of the previously solved subproblem and $t_{\text {SUB }}^{0}$ the mean duration if solving a subproblem from scratch. Thus with $L$ being the number of iterations,

$$
\frac{t_{s}}{L}=t_{\mathrm{MA}}+t_{\mathrm{SUB}}^{0}+\left(n_{\mathrm{prep}}+n\right) t_{\mathrm{SUB}}
$$

and

$$
\frac{t_{p}}{L}=\left\{\begin{array}{lll}
t_{\mathrm{MA}}+t_{\mathrm{SUB}}^{0}+\frac{\left({ }^{n} \text { prep }+n\right)}{p-1} t_{\mathrm{SUB}}, & \text { if } & n, n_{\mathrm{prep}} \geq p-1 \\
t_{\mathrm{MA}}+t_{\mathrm{SUB}}^{0}+\frac{(1+n)}{p-1} t_{\mathrm{SUB}}, & \text { if } & n \geq p-1, n_{\text {prep }}<p-1 .
\end{array}\right.
$$

If the sample size $n$ is smaller than the number of sub processors the parallel algorithm is not efficient because not all computer resources are utilized. Using the above formulae, we can compute the efficiency for the case of $n, n$ prep $\geq p-1$, for example, as

$$
E=\frac{t_{\mathrm{MA}}+t_{\mathrm{SUB}}^{0}+\left(n_{\mathrm{prep}}+n\right) t_{\mathrm{SUB}}}{p t_{\mathrm{MA}}+p t_{\mathrm{SUB}}^{0}+\frac{p}{p-1}\left(n_{\mathrm{prep}}+n\right) t_{\mathrm{SUB}}} .
$$

One can see that for a fixed number of processors the efficiency approaches $100 \%$ as the sample size increases. This is obvious because increasing the sample size means adding computational work that can be conducted in parallel. Thus the parallel implementation is most efficient when solving problems with large sample sizes. On the other hand one can also see that for a given sample size the efficiency decreases with increasing number of processors. The maximum number of processors that can be utilized meaningfully is $1+\max \left\{n_{\mathrm{prep}}, n\right\}$. 


\subsection{NUMERICAL RESULTS FOR THE PARALLEL IM- PLEMENTATION}

Experiments were conducted to validate the parallel implementation and to obtain measures of computing time, speedup and efficiency. Test problems taken from the literature are usually small with a small number of stochastic parameters. We tested our parallel processing methodology on a truly large-scale problem, BIGNEW, which is a modified version of the capacity expansion planning model WRPM, as already discussed in the previous results section. There are 11 stochastic parameters ( 8 stochastic availabilities of generators and transmission lines and 3 uncertain demands) with discrete distributions with 3 or 4 outcomes. While other implementations of WRPM cover up to 3 future time periods, BIGNEW covers a planning horizon of only one future time period and is formulated as a two-stage stochastic linear program with recourse. The problem is large-scale, though it is by far not the largest we have solved serially. The number of universe scenarios is about $10^{6}$; the equivalent deterministic formulation of the problem (if it were possible to state it explicitly) would have more than 0.3 billion constraints.

This test problem has been solved repeatedly using different numbers of processors and different sample sizes. The parallel implementation has been improved as we learned more about its characteristics. For example, we varied the sample size within the range 20 to 63 , where we always have at least as many processors at hand as there are subproblems to be solved in one parallel phase. Table 3.1 summarizes the results. The computing time (measured in CPU minutes per iteration) is approximately constant at a level of 0.12 minutes per iteration for sample size 20 up to 29. Then it jumps to a level of approximately $0.17 \mathrm{~min}$ per iteration, where it again remains approximately constant.

In the test example the number of preparatory subproblems to compute the importance distribution is 29 . Figure 3.2 shows how the algorithm parallelizes. It schematically shows busy and idle times for different processors with sample size 63 during the first two iterations. Note the two phases of solving subproblems:' the preparatory phase and the sample phase. In the preparatory phase only 29 subproblems have.to be solved, compared to 63 subproblems in the sample phase. Each optimization is started using the basis for the optimal solution of the problem previously solved on the same processor. At the beginning, all problems are started from scratch as no basis is available. In the first iteration processors 1 to 29 start from scratch in the preparatory phase but use the optimal bases from the preparatory subproblems in the sample phase. Processors 30 to 63 do not solve subproblems in the preparatory phase; thus the sample subproblems assigned to these processors are started from scratch.

Solving a subproblem from scratch takes considerably more time than solving it with a good starting basis (warm start). The master processor starts operation when all necessary subproblems are solved completely, both in the preparatory phase 
Table 3.1: Warm start all subs

\begin{tabular}{|l|c|c|c|c|c|c|c|c|}
\hline $\begin{array}{l}\text { nodes } \\
\text { reser. }\end{array}$ & $p$ & $n$ & iter & $\begin{array}{c}\text { CPU } \\
\text { (min) }\end{array}$ & time/it & $\begin{array}{c}\text { CPU } \\
\text { (min) }\end{array}$ & time/it & obj \\
\hline 32 & 32 & 20 & 66 & 7.898 & 0.120 & 7.898 & 0.120 & 188382 \\
32 & 32 & 24 & 63 & 7.502 & 0.119 & 7.502 & 0.119 & 188025 \\
32 & 32 & 26 & 61 & 7.866 & 0.129 & 7.866 & 0.129 & 188236 \\
32 & 32 & 27 & 56 & 6.219 & 0.111 & 6.319 & 0.111 & 188232 \\
32 & 32 & 28 & 52 & 6.434 & 0.124 & 6.434 & 0.124 & 188195 \\
32 & 32 & 29 & 60 & 7.303 & 0.122 & 7.303 & 0.122 & 188492 \\
32 & 32 & 30 & 64 & 11.173 & 0.175 & 7.770 & 0.121 & 188271 \\
32 & 32 & 31 & 60 & 10.767 & 0.179 & 7.331 & 0.122 & 188301 \\
64 & 33 & 32 & 64 & 12.409 & 0.194 & N/A & N/A & 188347 \\
64 & 36 & 35 & 59 & 10.334 & 0.175 & N/A & N/A & 188295 \\
64 & 41 & 40 & 63 & 10.898 & 0.173 & 7.516 & 0.119 & 188261 \\
64 & 51 & 50 & 63 & 11.034 & 0.175 & 7.528 & 0.119 & 188378 \\
64 & 61 & 60 & 70 & 12.035 & 0.172 & 8.374 & 0.120 & 188549 \\
64 & 64 & 63 & 75 & 12.645 & 0.169 & 8.821 & 0.118 & 188492 \\
\hline
\end{tabular}

and the sampling phase. The computing time in each phase is determined by the maximum duration spent for solving a subproblem. In the first iteration processors 30 to 63 are idle during the preparation phase and solve subproblems from scratch in the sample phase; the maximum time spent in the sample phase by these processors is much larger than the maximum time spent by processors 1 to 29 . The duration of the sample phase in the first iteration is therefore much larger for sample sizes larger than 29 , the number of preparatory subproblems. The jump in the computing time at sample size 30 is due to this effect.

Besides the impact of the starting basis in the first iteration, there is also an impact in all other iterations. A basis from the optimal solution of a subproblem of the current iteration is expected to be a better starting basis than a basis from the optimal solution of a subproblem of the previous iteration. Note that the effect only

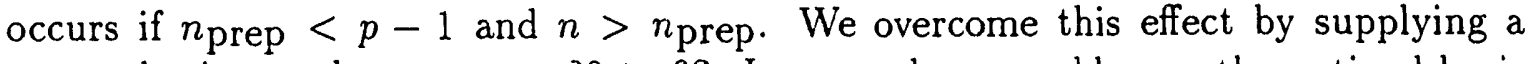
proper basis to sub processors 30 to 63 . In general one could copy the optimal basis of the subproblem that has finished first in the preparatory phase to processors 30 to 63 to warm start all subproblems in the sample phase. As idle processors are not used for any other tasks and cannot be used in a timesharing mode by other users it is more efficient (as no communication is necessary) to assign an arbitrary preparatory subproblem (e.g. subproblem 1) also to processors 30 to 63 and solve it on each of the processors to have the optimum starting basis ready for the sampling phase. Table 3.1 also shows the results for warm starting all subs. The computing time remains approximately constant over the whole range of sample sizes. The results show that 


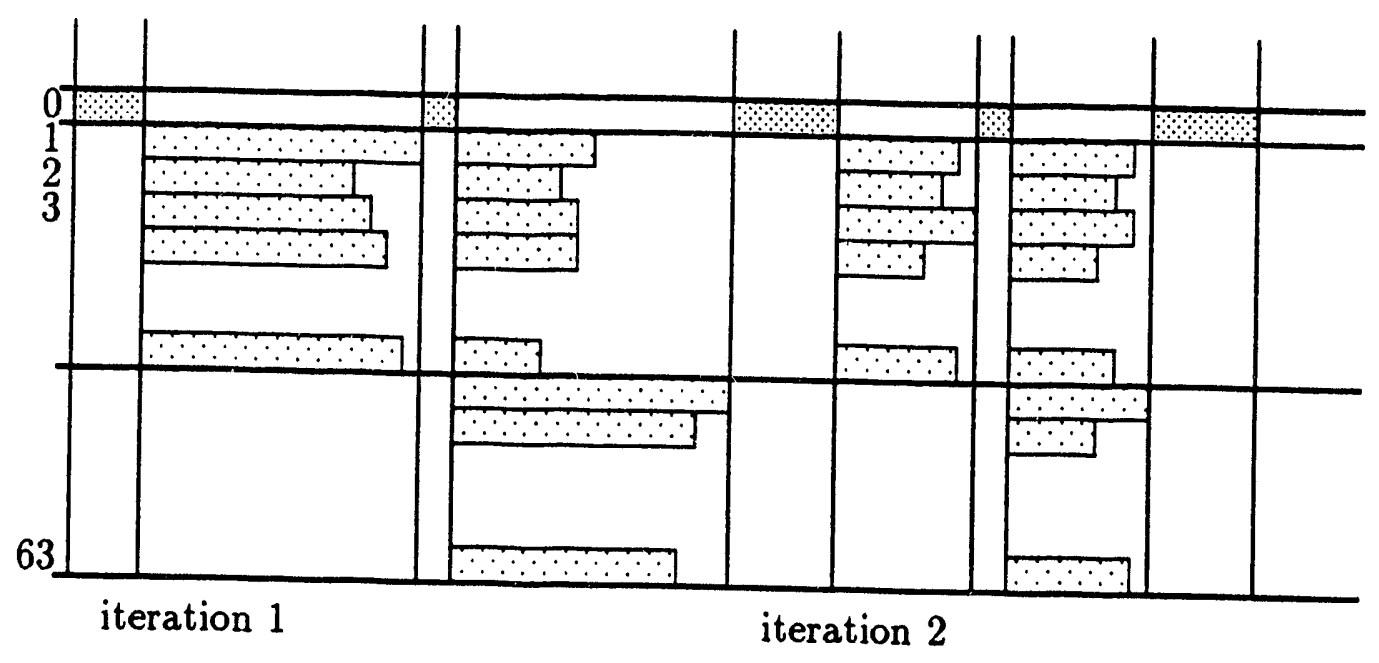

Figure 3.2: The parallel algorithm

the effect of the jump in the computing time is completely compensated. No time differences resulting from $n_{\text {prep }}<n$ can be observed. Thus the model for determining the parallel time $t_{p}$ is valid for all numbers of preparatory problems $n_{\text {prep. }}$.

The analysis so far has concerned previous implementations where the assignment of subproblems to sub processors was hardwired. In our current implementation, subproblems are sent to the next idle node. This implementation allows for any number of subproblems $n_{\text {prep }}$ and $n$ per iteration and divides up the number of subproblems efficiently among the processors available. If necessary the warm start procedure is used. In the following we are interested in the efficiency of the method with respect to both the sample size and the number of processors.

For determining the efficiency we use the formulae developed in the previous section. Varying the sample size over a sufficiently large range, we estimate the parameters for determining the computing time. Table 3.2 gives results for sample sizes from 100 to 600 using 64 processors representing the parallel computation time versus the sample size for both the actual time measurements and the estimates from the formulae. One can see that the algebraic formulae give an excellent estimate of the actual parallel computing time. We estimate $t_{\mathrm{MA}}+t_{\mathrm{SUB}}^{0}$ to be 0.0962 and $t_{\mathrm{SUB}}$ to be 0.0149 . Using these parameters we compute the corresponding serial time $t_{s}$, the speedup $S$ and the efficiency $E$, which are also reported in Table 3.2. While the efficiency is low for small sample sizes it rapidly improves with increasing sample size. In the case of sample size 600 , we obtain a speedup of about 37.5 , which means that with 64 processors we reduce the computation tirne by a factor of 37.5 . The total 
Table 3.2: Speedup and Efficiency

\begin{tabular}{|c|c|c|c|c|c|c|}
\hline$n$ & iter & $t_{p}$ & $t_{p}$ & $\overline{t_{s}}$ & $S$ & $E$ \\
\hline & & actual & $\begin{array}{l}\text { est. by } \\
\text { formula }\end{array}$ & & speedup & efficiency \\
\hline 100 & 63 & 0.132 & 0.135 & 2.024 & 14.99 & 23.456 \\
\hline 200 & 72 & 0.159 & 0.159 & 3.519 & 22.13 & 34.674 \\
\hline 300 & 76 & 0.182 & 0.182 & 5.014 & 27.55 & 42.973 \\
\hline 400 & 84 & 0.213 & 0.206 & 6.508 & 31.59 & 49.360 \\
\hline 500 & 69 & 0.229 & 0.230 & 8.003 & 34.80 & 54.428 \\
\hline 600 & 69 & 0.250 & 0.253 & 9.497 & 37.54 & 58.547 \\
\hline
\end{tabular}

parallel time is 17.3 minutes while in a serial implementation the time to solve the problem would be 652 minutes. Figure 3.3 shows the dependency of the efficiency upon the sample size when 64 processors are used.

Using estimates based on the formulae for the parallel time, we compute the efficiency as a function of the number of processors used. Figure 3.4 gives a graphical representation. For small numbers of processors the effect of only $p-1$ processors operating in parallel when using $p$ processors dominates the result. For example when using 2 processors we switch between the master processor and only one sub processor. There is no parallel overlapping in the computation. In this case we perform a serial computation distributed to 2 processors. The efficiency hence is $50 \%$. The efficiency increases until the above-mentioned effect is no longer dominating. For example, for sample size 600 and 12 processors, the efficiency is about $82 \%$. The efficiency decreases with increasing numbers of processors beyond 12 . Using 64 processors, we obtain an efficiency of $58.54 \%$ when the sample size is 600 .

For the runs documented in Table 3.2, Table $3.3 \mathrm{r}$ rts on the optimum objective function value and the $95 \%$ confidence interval. The lower bound distributions have less variance than the upper bound distributions; hence the confidence interval is asymmetric. Using a sample size of 100 (out of about 1 million universe scenarios) we obtain an optimal solution of 188348.7 with a $95 \%$ confidence interval of $0.08 \%$ on the lower side and $0.018 \%$ on the upper side. Even with only small sample sizes we obtain highly accurate results. The parallel time to run the problem was 8.3 minutes on the Hypercube.

The optimal objective function value remains stable when increasing the sample size. That again shows that we obtained good estimates. The confidence interval decreases with increasing sample size and the rate of $n^{-0.5}$ is verified by the computational results.

Using a sample size of 600 we obtain an optimal objective function value of 188351.8 with a $95 \%$ confidence interval of $0.04 \%$ on the left side and $0.06 \%$ on 
efficiency (\%)

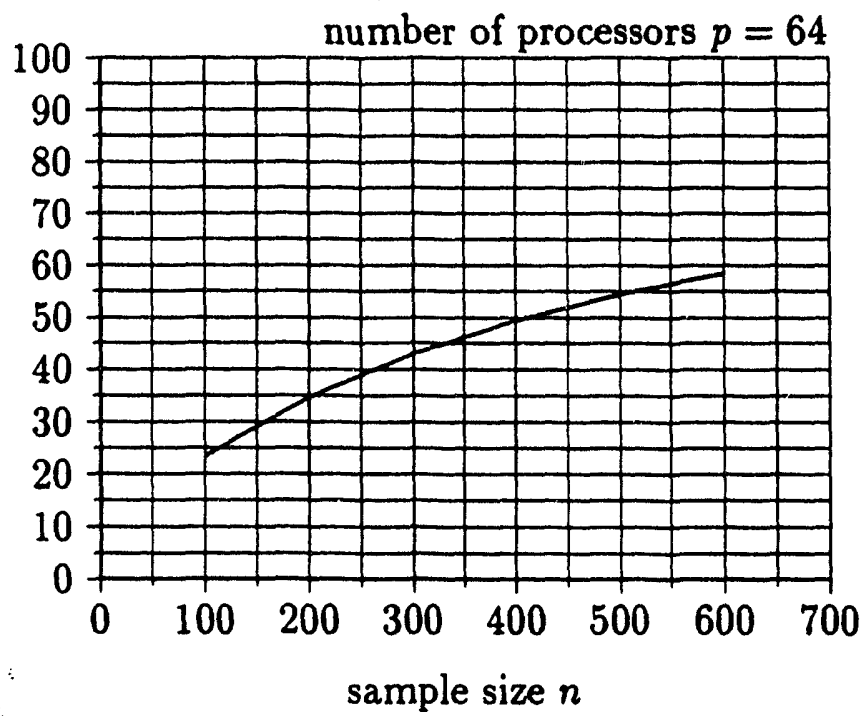

Figure 3.3: Efficiency versus sample size

the right side. Thus the optimal solution with $95 \%$ confidence satisfies $188276.7 \leq$ $z^{*} \leq 1884730$. All solutions reported in Table 3.3 fall within this range. The computation time on the Hypercube was 17.3 minutes. It is interesting to note that during the process of solving the problem about 43400 subproblems with 289 rows and 302 columns each and 69 master problems were solved. 


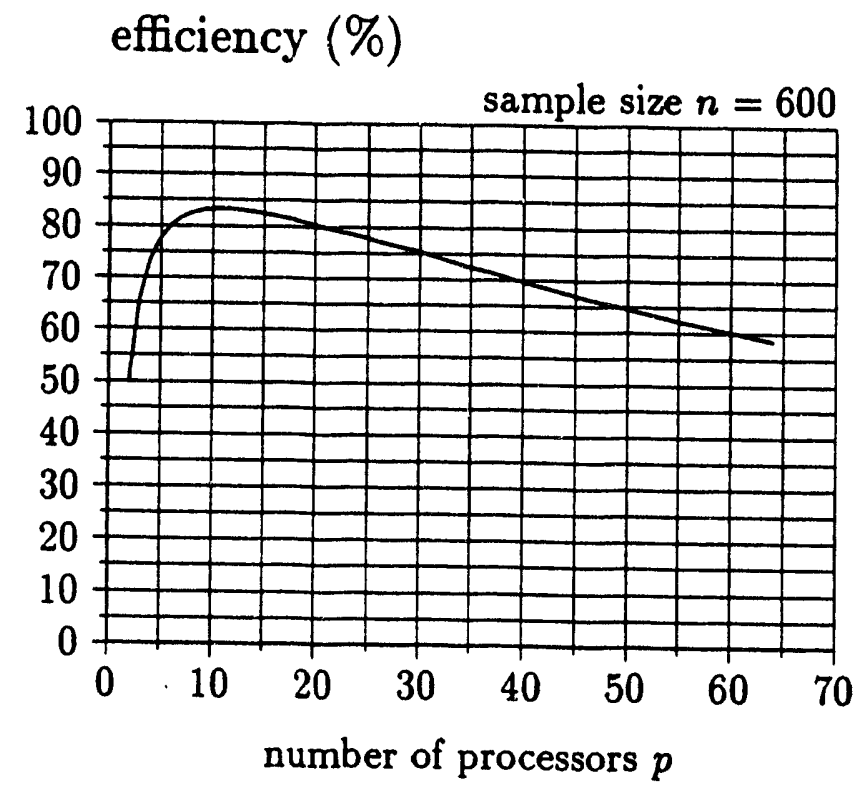

Figure 3.4: Efficiency versus number of processors

Table 3.3: Optimal Solution

\begin{tabular}{|c|c|c|c|c|c|c|c|}
\hline & & & \multicolumn{4}{|c|}{$95 \%$ confidence interval } & \\
\hline$n$ & iter & obj & lower & upper & total & $\%$ & CPU \\
\hline & & & & & $\begin{array}{c}\text { lower } \\
\text { tupper }\end{array}$ & of obj & $(\min )$ \\
\hline 100 & 63 & 188348.7 & 153.0 & 344.4 & 497.4 & 0.26 & $\overline{8.3}$ \\
\hline 200 & 72 & 188390.9 & 144.8 & 161.8 & 306.6 & 0.16 & 11.4 \\
\hline 300 & 76 & 188344.9 & 100.5 & 180.2 & 280.7 & 0.15 & 13.8 \\
\hline 400 & 84 & 188328.4 & 79.9 & 153.7 & 233.5 & 0.12 & 17.9 \\
\hline 500 & 69 & $188:$ & 78. & 131.1 & 209.0 & 0.11 & 15.8 \\
\hline 600 & 69 & 188351.8 & 75.1 & 121.2 & 196.3 & 0.10 & 17.3 \\
\hline
\end{tabular}




\section{Techniques for Solving Multi-Stage Problems}

\subsection{MULTI-STAGE STOCHASTIC LINEAR PROGRAMS}

The use of stochastic programming techniques for solving large dynamic systems under uncertainty has been hampered until recently by the sheer size of the problems constructed when they are restated as deterministic linear programs. To solve them it has been necessary to keep the number of scenarios representing uncertainties fairly small. Only a few attempts have been made to solve practical multi-stage decision models whose future events are spread over several periods. In this section we extend the methodology developed for solving two-stage problems to multi-stage problems.

Multi-stage planning problems can often be formulated as linear programs with a dynamic matrix structure which, in the deterministic case, appears in a staircase pattern of blocks formed by non-zero sub-matrices. These blocks correspond to and are different for different time periods. System (4.1) below shows the structure of a dynamic linear program of $T$ periods.

$$
\begin{aligned}
& \begin{aligned}
\min z & = \\
c_{1} x_{1} & +c_{2} x_{2}+\cdots+c_{T-1} x_{T-1}+c_{T} x_{T}
\end{aligned} \\
& \mathrm{~s} / \mathrm{t} \\
& A_{1} x_{1} \quad=b_{1} \\
& \begin{array}{ccc}
-B_{1} x_{1}+A_{2} x_{2} & & b_{2} \\
& \ddots & \vdots
\end{array} \\
& -B_{T-1} x_{T-1},+A_{T} x_{T}=b_{T} \\
& x_{1}, x_{2}, \ldots, \quad x_{T-1} \quad, \quad x_{T} \geq 0 .
\end{aligned}
$$

In the stochastic case, the blocks of coefficients and right-hand sides in different time periods are functions of several parameters whose values vary stochastically according to dependent and independent distributions, which we assume to be known. The resulting problem is a multi-stage stochastic linear program. Even for a problem with a small number of stochastic parameters per stage, the size of the multi-stage problem, when expressed in equivalent deterministic form, can get so large as to appear intractable. 
The multi-stage stochastic extension of a deterministic dynamic linear program can be formulated as follows:

$$
\begin{aligned}
& \begin{aligned}
\min z & = \\
c_{1} x_{1} & +E\left(c_{2} x_{2}^{\omega_{2}}+\cdots+E\left(c_{T-1} x_{T-1}^{\omega_{T-1}, \ldots, \omega_{2}}+E\left(c_{T} x_{T}^{\omega_{T}, \ldots, \omega_{2}}\right)\right)\right)
\end{aligned} \\
& \mathrm{s} / \mathrm{t} \\
& A_{1} x_{1} \\
& -B_{1}^{\omega_{2}} x_{1}+A_{2} x_{2}^{\omega_{2}} \\
& \cdot \\
& -B_{T-1}^{\omega_{T}} x_{T-1}^{\omega_{T-1}, \ldots, \omega_{2}}+A_{T} x_{T}^{\omega_{T}, \ldots, \omega_{2}}=b_{T}^{\omega_{T}} \\
& x_{1}, \quad x_{2}^{\omega_{2}} \quad, \ldots, \quad x_{T-1}^{\omega_{T-1}, \ldots, \omega_{2}} \quad, \quad x_{T}^{\omega_{T}, \ldots, \omega_{2}} \geq 0 \\
& \omega_{t} \in \Omega_{t}, t=2, \ldots, T \text {. } \\
& =b_{1} \\
& =b_{2}^{\omega_{2}}
\end{aligned}
$$

While the first-stage parameters $c_{1}, A_{1}, b_{1}$ are known to the planner with certainty, the parameters of stages $2, \ldots, T$ are assumer to be known only by their distributions. We consider uncertainty in the coefficients of the transition matrices $B_{t}^{\omega_{t}}, t=1, \ldots, T-1$ and of the right-hand sides $b_{t}^{\omega_{t}}, t=1, \ldots, T$. We assume the coefficients of the technology matrices $A_{t}, t=1, \ldots, T$, and the objective function coefficients $c_{t}, t=2, \ldots, T$, to be known with certainty. The assumption of deterministic technology matrices and objective function coefficients 'uses the presentation but is not crucial to the solution method, which we have developed. The goal of the planner is to minimize the expected value of present and future costs.

In a general model, one has to allow the stochastic parameters of the transition matrices $B_{t}^{\omega_{t}}$ and the right-hand sides $b_{t}^{\omega_{t}}$ to be dependent both within a certain stage and between stages. In the latter case the distributions of the stochastic parameters in poriod $t$ depend on the outcomes of the stochastic parameters in peris $t t-1$.

The underlying "wait-and-see" decision-making process is as follows: The decision maker makes a first-stage iecision $\hat{x}_{1}$ before observing any outcome of the random parameters. Then he wait; ur $: 1$ an outcome of the second-ctage random parameters is realized. The second-stage lecision is then made based on the knowledge of the realization $1 v_{2}$ but without observing the outcome of any random parameters of stages $3, \ldots, T$, and so forth. As the state (the actual outcome) is carried forward to the following period, the decision tree grows exponentially with the number of stages. The corresponding decision tree is given in Figure 4.1. The figure represents the exir.r le of a 1 ir-stage problem with only 3 outcomes per stage.

e consider discrete distributions of random parameters with a finite number of oute smes, e.g., $\omega_{t} \in \Omega_{t}, \Omega_{t}=\left\{1, \ldots, K_{t}\right\}, t=1, \ldots, T$. With $K_{t}$ being the number of scenarios in period $t$, the total number of scenarios for all $T$ stages is $\prod_{t=1}^{T} K_{t}$. The number $K_{t}$ is expectr d to be large, as it is derived from crossing the sets of possible outcomes of the different random parameters within a period. For example, if the 


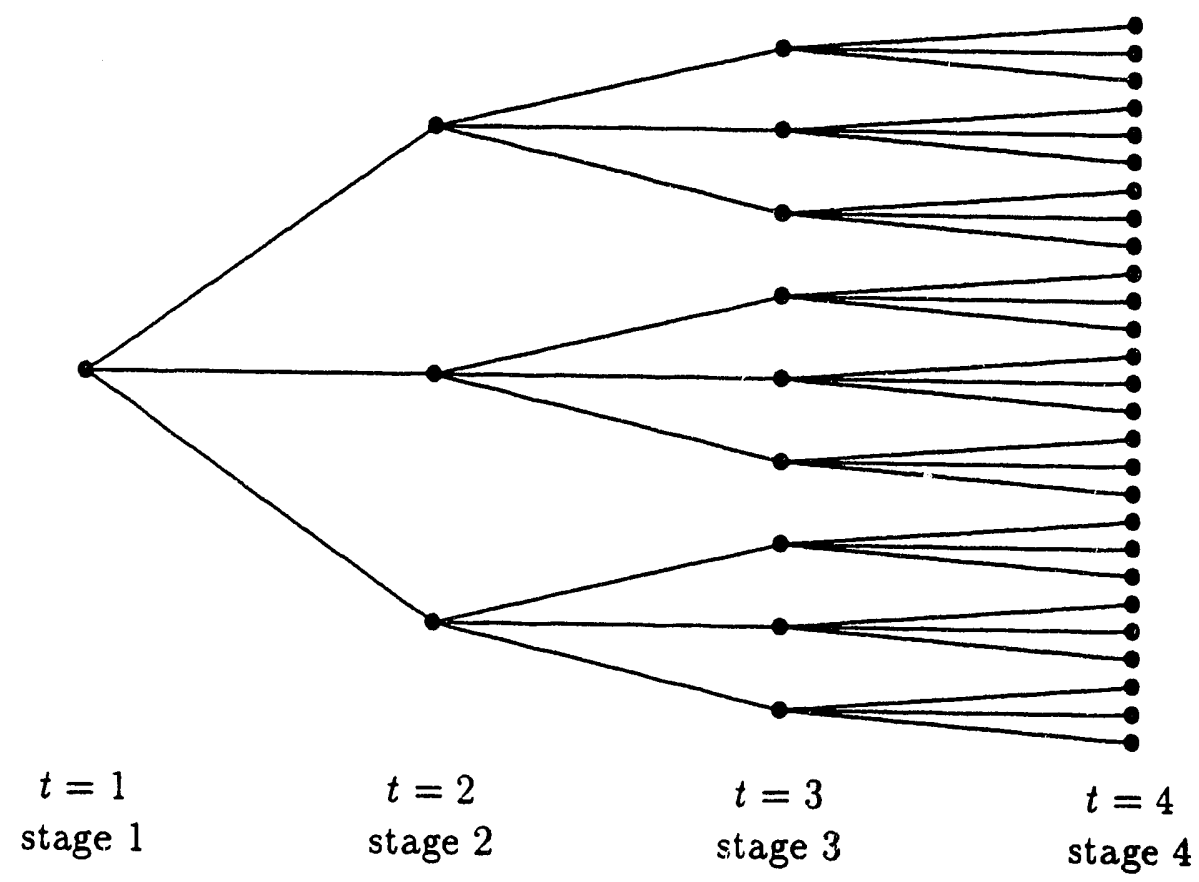

Figure 4.1: Decision tree of a general class of "wait and see" multi-stage problems

dimension of the random vector in period $t$ is $h_{t}$ and $\Omega_{t}^{j}$ contains $k_{t}^{j}$ elements, then $K_{t}=\prod_{j=1}^{h_{t}} k_{j}^{t}$.

For example, in the hydro power control problem of Section 4.8.1, consider the case of prediction error in 10 inflows, modeled as independent random parameters with 3 outcomes each: the number of scenarios per period is $3^{10} \approx 60,000$. If we consider only 3 periods of "wait and see" type planning ahead and neglect uncertainties of periods further ahead, then the total number of scenarios that have to be examined is in the order of $10^{9}$. As another example, in the asset allocation problem of Section 4.8.2, consider the case of 20 independent factors (used to describe the returns of, say, 500 assets) modeled as random parameters with 5 outcomes each: the number of scenarios per period is $5^{20} \approx 10^{14}$. If there are only 3 periods, then the total number of scenarios grows to $10^{28}$. The dimensions of an equivalent linear program of an asset allocation problem with a universe of about 500 assets is approximately $5 \cdot 10^{30}$ rows and $1.5 \cdot 10^{31}$ columns. It is of course impossible to write down this linear program explicitly. 


\subsection{DEPENDENCY AMONG STOCHASTIC PARAME- TERS}

We model dependency between stochastic parameters both within a certain stage and between stages. We refer to the former kind of dependency as intra-stage dependency and to the latter as inter-stage dependency.

We define the vector $W_{t}$ to be the vector of all dependent random parameters of stage $t$. $W_{t}$ contains both the random parameters in the transition matrix $B_{t-1}$ and the random parameters of the right-hand side $b_{t}$. We will use the notation

$$
W_{t}=\operatorname{vec}\left(B_{t-1}, b_{t}\right)
$$

to express that all random parameters in the transition matrix $B_{t-1}$ and all random parameters in the right-hand side $b_{t}$ are organized in the random vector $W_{t}$. Each component of $W_{t}$ refers uniquely to an element of the matrix $B_{t-1}$ or to an element of the vector $b_{t}$. We define inverse operations of $W_{t}=\operatorname{vec}\left(B_{t-1}, b_{t}\right)$ as

$$
B_{t-1}\left(W_{t}\right)=\operatorname{vec}_{B}^{-1}\left(W_{t}\right)
$$

and

$$
b_{t}\left(W_{t}\right)=\operatorname{vec}_{b}^{-1}\left(W_{t}\right)
$$

to express that, given the random vector $W_{t}$, the corresponding random elements in $B_{t-1}$ and $b_{t}$ are uniquely determined. With $m_{t+1} \times n_{t}$ being the dimensions of the transition matrices $B_{t}$ and $m_{t}$ being the dimension of the right-hand side $b_{t}$, the dimension of the dependent random vector $W_{t}$ is at most $m_{t+1} \times n_{t}+m_{t+1}$. We denote the dimension of $W_{t}$ with $\tilde{h}_{t}$, thus $W_{t}=\left(W_{1, t}, \ldots, W_{\tilde{h}_{t}, t}\right)$. With respect to the solution procedure discussed later we formulate a special kind of dependency model which is based on linear intra-stage and inter-stage dependency.

\subsubsection{Intra-Stage Dependency}

We describe correlation of random parameters within a stage by a linear relation, i.e. outcomes of the $\tilde{h}_{t}$ uncertain dependent parameters $\left(W_{1, t}, \ldots, W_{\tilde{h}_{t}, t}\right)$ can be obtained by multiplying outcomes of a vector of independent random parameters $V_{t}=\left(V_{1, t}, \ldots, V_{h_{t}, t}\right)$ by a matrix $\tilde{\beta}_{t}\left(\tilde{h}_{t} \times h_{t}\right)$,

$$
W_{t}=\tilde{\beta}_{t} V_{t} .
$$

We denote an outcome of the independent random vector $V_{t}$ by $v_{t}$ or $v_{t}^{\omega_{t}}$, where $\omega_{t} \in \Omega_{t}$. We denote an outcome of an individual random parameter in period $t$, say $V_{t}^{j_{t}}$, by $v_{t}^{j_{t}}$, also denoted as $v^{j_{t}, \omega_{t}^{j}}$, where $\omega_{t}^{j_{t}} \in \Omega_{t}^{j_{t}}$, with corresponding probability $p\left(v_{t}^{j_{t}}\right)=\operatorname{prob}\left(V_{t}^{j_{t}}=v_{t}^{j_{t}}\right)$. The set of all possible outcomes in stage $t$ is constructed by crossing the sets of possible outcomes of the components of $V_{t}, \Omega_{t}^{j t}, j_{t}=1, \ldots, h_{t}$ as $\Omega_{t}=\Omega_{t}^{1} \times \Omega_{t}^{2} \times \cdots \times \Omega_{t}^{h_{t}}$. Because of the independence of the individual random 
parameters of $V_{t}, p\left(v_{t}\right)=p\left(v_{t}^{1}\right) p\left(v_{t}^{2}\right) \cdots p\left(v_{t}^{h_{t}}\right)$. The corresponding outcomes of the dependent random vectors $W_{t}$ are denoted by $w_{t}$ or $w_{t}^{\omega_{t}}$.

The linear intra-stage dependency model generates dependent scenarios based on random variations of independent random parameters. This property will become particularly important when implementing sampling for generating scenarios. In many applications the number of independent random parameters per stage, $h_{t}$, is expected to be much smaller than the number of dependent random parameters, $\tilde{h}_{t}$.

\subsubsection{Inter-Stage Dependency}

For expository purposes we consider an equal number of independent random parameters in each of the different stages and denote this number as $h$. We consider inter-stage dependency as a Markovian process (linear dependency).

$$
w_{t}^{\omega_{t}, \ldots, \omega_{2}}=\beta_{0} v_{t}^{\omega_{t}}+\beta_{1} w_{t-1}^{\omega_{t-1}}+\cdots+\beta_{t-2} w_{2}^{\omega_{2}} .
$$

The matrices $\beta_{0}, \beta_{1}, \ldots, \beta_{T-2}$ are matrices of dimension $(h \times h)$. If they are diagonal matrices the value of an outcome of the random parameter $W_{t}^{i}$ is a weighted sum of some independent random variation in period $t$, denoted by $v_{t}^{i}$ and the values of the observed outcomes in the previous periods $t-1, t-2, \ldots, T-2$. If the matrices $\beta_{0}, \beta_{1}, \ldots, \beta_{T-2}$ are non-diagonal matrices, the outcome of each element in $W_{t}$ is dependent upon the outcomes of all elements in $W_{t-1}, W_{t-2}$ back to $W_{2}$ and the independent random variations of $V_{t}$. Given observed outcomes of $W_{t-1}, W_{t-2}$ back to $W_{2}$ one can easily generate a scenario of $W_{t}$ by independent random variations of $V_{t}$. One can see that a scenario in period $t$ is determined based on the observed historical outcomes of the dependent random parameters and by the variations of the independent random parameters in period $t$. We refer to our linear dependency model as an auto-correlative model. The class of auto-correlative models is a wide class of dependency models that is well studied in the literature. Many different applications in practice can be formulated using the auto-correlative approach. The parameters of the auto-correlative model can be estimated based on historical observations of the outcomes of the stochastic parameters. We can now state the auto-correlative model in the notation of the formulation of the multi-stage stochastic linear program:

$$
\begin{gathered}
B_{t-1}\left(w_{t}^{\omega_{t}, \ldots, \omega_{2}}\right)=\operatorname{vec}_{B}^{-1}\left(\beta_{0} v_{t}^{\omega_{t}}\right)+\sum_{\tau=1}^{t-2} \operatorname{vec}_{B}^{-1}\left(\beta_{\tau} \operatorname{vec}\left(B_{t-\tau-1}^{\omega_{t-\tau}}, b_{t-\tau}^{\omega_{t-\tau}}\right)\right) \\
b_{t}\left(w_{t}^{\omega_{t}, \ldots, \omega_{2}}\right)=\operatorname{vec}_{b}^{-1}\left(\beta_{0} v_{t}^{\omega_{t}}\right)+\sum_{\tau=1}^{t-2} \operatorname{vec}_{b}^{-1}\left(\beta_{\tau} \operatorname{vec}\left(B_{t-\tau-1}^{\omega_{t}-\tau}, b_{t-\tau}^{\omega_{t-\tau}}\right)\right)
\end{gathered}
$$

While the general auto-correlative model fully applies to all the discussions that follow, we will additionally present a special case of the auto-correlative model where we make assumptions in order to simplify the presentation. We treat the random matrices $B_{t-1}$ and the random right-hand sides $b_{t}$ separately. That means we assume 
that either the transition matrices $B_{t-1}$ are random or that the right-hand sides $b_{t}$ are random, but not both at the same time. We consider further instead of matrices $\beta_{\tau}, \tau=0, \ldots, T-2$, scalars $\beta_{\tau}^{B}, \tau=0, \ldots, T-2$, for modeling dependency of elements of the transition matrices $B_{t-1}$ or scalars $\beta_{\tau}^{b}, \tau=0, \ldots, T-2$, for modeling dependency of elements of the right-hand sides $b_{t}$. With these assumptions the simplified linear dependency model can now be formulated in the case of randomness in the transition matrices as:

$$
B_{t-1}^{\omega_{t}, \ldots, \omega_{2}}=\beta_{0}^{B} \tilde{\eta}_{t}^{\omega_{t}}+\beta_{1}^{B} B_{t-2}^{\omega_{t-1}}+\beta_{2}^{B} B_{t-3}^{\omega_{t-2}}+\ldots+\beta_{t-2}^{B} B_{1}^{\omega_{2}},
$$

or in the case of randomness in the right-hand sides as:

$$
b_{t}^{\omega_{t}, \ldots, \omega_{2}}=\beta_{0}^{b} \eta_{t}^{\omega_{t}}+\beta_{1}^{b} b_{t-1}^{\omega_{t-1}}+\beta_{2}^{b} b_{t-2}^{\omega_{t-2}}+\ldots+\beta_{t-2}^{b} b_{2}^{\omega_{2}} .
$$

In this formulation $\tilde{\eta}_{t}$ represents a matrix of the same dimensions as $B_{t-1}$ whose elements are functions of random parameters which are independent of the random parameters of all previous periods, and $\eta_{t}$ represents a vector of the dimension of $b_{t}$ whose elements are functions of random parameters which are independent of the random parameters of all previous periods.

\subsection{DUAL DECOMPOSITION}

A description of how Benders (1962) [6] decomposition algorithm can be applied to solve two-stage stochastic linear programs can be found in Van Slyke and Wets (1969) [122]. Birge (1985) [9] extended the two-stage concept for solving multi-stage stochastic linear programs by using a nested Benders decomposition scheme.

Using dual decomposition we decompose the problem into subproblems for different stages $t$. In the most general case, in which there is a dependency of stochastic parameters between stages, the number of subproblems is equal to the number of scenarios in each stage $t$. To distinguish one subproblem from another, each is indexed with $\omega_{t}, \ldots, \omega_{2}$, where $\omega_{t}$ is the random event in stage $t$ and $\omega_{t-1}, \ldots, \omega_{2}$ is the path of previous events which gave rise to the particular subproblems in stage $t$.

For expository purposes, we assume initially that the random events occuring in one stage are independent of those that happened in the previous stage. In the independent case, scenarios $\omega_{t+1} \in \Omega_{t+1}$ in period $t+1$ are identical for each scenario $\omega_{t} \in \Omega_{t}$ in period $t$. The history is only carried forward through optimal decisions $\hat{x}_{t-1}^{\omega_{t-1}, \ldots, \omega_{2}}$ from previous periods.

The idea of using dual decomposition is to express in each stage $t$, and scenario $\omega_{t}$, the expected future costs (the impact of stages $t+1, \ldots, T$ ) by a scalar $\theta_{t}$. The value of $\theta_{t}$ is constrained by a set of "cuts", which are necessary conditions for feasibility and optimality expressed solely in terms of the stage $t$ decision variables $x_{t}$ and $\theta_{t}$. Cuts are initially absent and then sequentially added to the stage $t$ problems. Each scenario subproblem collects the information about expected future costs by means of the cuts. 
The relation between the different stages and scenarios in the decomposed multistage problem is summarized as follows:

\section{The Stage 1 Problem:}

$$
\begin{aligned}
& \min z_{1}=c_{1} x_{1}+\theta_{1} \\
& \mathrm{~s} / \mathrm{t} \\
& \begin{array}{rrr}
\pi_{1}: & A_{1} x_{1} & =b_{1} \\
\rho_{1}^{l_{1}}: & -G_{1}^{l_{1}} x_{1}+\alpha_{1}^{l_{1}} \theta_{1} & \geq g_{1}^{l_{1}}, \quad l_{1}=1, \ldots L_{1}
\end{array} \\
& x_{1} \quad \geq 0 \text {. }
\end{aligned}
$$

The Stage $t, t=2, \ldots, T-1$, Problems:

$$
\begin{aligned}
\min z_{t}^{\omega_{t}}=c_{t} x_{t}^{\omega_{t}}+\theta_{t}^{\omega_{t}} & \\
\mathrm{~s} / \mathrm{t} & \\
\pi_{t}^{\omega_{t}}: & A_{t} x_{t}^{\omega_{t}} \\
\rho_{t}^{l_{t}, \omega_{t}}: & =b_{t}^{\omega_{t}}+B_{t-1}^{\omega_{t}} \hat{x}_{t-1} \\
-G_{t}^{l_{t}} x_{t}^{\omega_{t}}+\alpha_{1}^{l_{1}} \theta_{t}^{\omega_{t}} & \geq g_{t}^{l_{t}}, \quad l_{t}=1, \ldots, L_{t} \\
x_{t}^{\omega_{t}} & \geq 0 .
\end{aligned}
$$

The Stage T Problem:

$$
\begin{aligned}
\min z_{T}^{\omega_{T}}= & c_{T} x_{T}^{\omega_{T}} \\
\mathrm{~s} / \mathrm{t} & \\
\pi_{T}^{\omega_{T}}: & A_{T} x_{T}^{\omega_{T}}=b_{T}^{\omega_{T}}+B_{T-1}^{\omega_{T}} \hat{x}_{T-1} \\
& x_{T}^{\omega_{T}} \geq 0 .
\end{aligned}
$$

$\min z_{1}$ represents the optimal objective function value in the first stage, and $x_{1}, \theta_{1}$ represent the optimal solution. The vector $\pi_{1}$ denotes the optimal dual prices associated with the original first-stage constraints, and the scalars $\rho_{1}^{l_{1}}$ are the optimal dual prices associated with the cuts, which have been added so far in decomposition iterations $l_{1}=1, \ldots, L_{1}$. In later stages, the optimal objective function values $\min z_{t}^{\omega_{t}}=\min z_{t}^{\omega_{t}}\left(\hat{x}_{t-1}\right)$, the optimal dual prices $\pi_{t}^{\omega_{t}}=\pi_{t}^{\omega_{t}}\left(\hat{x}_{t-1}\right)$ associated with the original stage $t$ constraints, and the optimal dual prices $\rho_{t}^{l_{t}, \omega_{t}}=\rho_{t}^{l_{t}, \omega_{t}}\left(\hat{x}_{t-1}\right)$ associated with the cuts are all dependent upon $\hat{x}_{t-1}$, the optimal solution passed as input from the previous stage $t-1$. According to the scenario evolution in the previous stages, an optimal solution $\hat{x}_{t-1}$ is actually indexed by the scenario outcomes of all previous stages and is therefore denoted as $\hat{x}_{t-1}^{\omega_{t-1}, \ldots, \omega_{2}}$. For the sake of exposition, we suppress the scenario history and present the optimal solution of subproblems in stage $t$, scenario $\omega_{t}$ as a function of the input $\hat{x}_{t-1}$.

We compute the expected future costs as

$$
z_{t+1}=E_{\omega_{t+1}} z_{t+1}^{\omega_{t+1}}
$$


the right-hand sides of the cuts as

$$
g_{t}^{l_{t}}=E_{\omega_{t+1}}\left(\pi_{t+1}^{\omega_{t+1}} b_{t+1}^{\omega_{t+1}}+\sum_{l_{t+1}=1}^{L_{t+1}} \rho_{t+1}^{l_{t+1}, \omega_{t+1}} g_{t+1}^{l_{t+1}}\right)
$$

and the coefficients of the cuts as

$$
G_{t}^{l_{t}}=E_{\omega_{t+1}} \pi_{t+1}^{\omega_{t+1}} B_{t}^{\omega_{t+1}}
$$

where

$$
\rho_{T}^{\omega_{T}}=0, G_{T}^{\omega_{T}}=0, \text { and } g_{T}^{\omega_{T}}=0
$$

A subproblem in stage $t$ and in scenario $\omega_{t}$ interacts with its predecessors and descendants by passing cuts backward and optimal solutions forward. Dual decomposition splits the multi-stage problem into a series of two-stage relations which are connected overall by a nesting scheme. We call the stage $t$, scenario $\omega_{t}$ problem the current master problem. It receives from its ancestor in period $t-1$ a solution $\hat{x}_{t-1}$. The current scenario is determined by the outcome $\omega_{t}$ of the random parameters in stage $t$, which are reflected in the right- hand side $b_{t}^{\omega_{t}}+B_{t-1}^{\omega_{t}} \hat{x}_{t-1}$. As stated above, $\hat{x}_{t-1}$ imbeds a history. This history has to be considered when nesting several stages. Given and subject to $\hat{x}_{t-1}$, we solve the stage $t$ problem in scenario $\omega_{t}$ and pass the obtained solution $\hat{x}_{t}^{\omega_{t}}$ to the descendant problems. By solving all problems $\omega_{t+1} \in \Omega_{t+1}$ (referred to as the universe case), we can compute exactly the expected value of the descendant stage costs $z_{t+1}=E_{\omega_{t}+1} z_{t+1}^{\omega_{t+1}}$ and the coefficients $G_{t}=E_{\omega_{t+1}} \pi_{t+1}^{\omega_{t+1}} B_{t+1}^{\omega_{t+1}}$ and right-hand side $g_{t}=E_{\omega_{t+1}}\left(\pi_{t+1}^{\omega_{t+1}} b_{t+1}^{\omega_{t+1}}+\sum_{l_{t+1}}^{L_{t+1}} \rho_{t+1}^{l_{t+1}, \omega_{t+1}} g_{t+1}^{l_{t+1}}\right)$ of a cut. The cut is added to the current master problem (stage $t$, scenario $\omega_{t}$ ), and by solving this problem again, another trial solution is obtained.

The optimal trial solution of the current master problem in stage $t$, scenario $\omega_{t}$ gives a lower bound, and the expected cost of this trial solution gives an upper bound for the expected costs of all scenarios descendant from stage $t$ scenario $\omega_{t}$. If the lower upper bounds are sufficiently close, the current master problem is deemed to represent adequadly the future expected costs and contains (by means of a sufficient number of cuts) all the information needed from future scenarios. In this case we say the current master is balanced with its descendant problems.

Note that the current master problem represents the expected future costs only subject to the trial solution $\hat{x}_{t-1}$, which was passed from its ancestor, and subject to the current scenario $\omega_{t}$. Furthermore we have implicitly assumed that the descendant problems in stage $t+1$ are also balanced with their descendant problems. Note, however, that the solution of the current stage $t$ scenario $\omega_{t}$ problem gives a lower bound for the expected costs of all descendant scenarios regardless of having collected a sufficient number of cuts. We shall exploit this fact. 


\subsection{PROPERTIES OF THE CUTS}

First we discuss various properties of cuts in the case of independence of stochastic parameters between stages. Then we extend the discussion to the case of Markovian dependency and restate the derived properties for the dependent case.

\subsubsection{The Case of Independence of Stochastic Parameters Between Stages}

The following properties of cuts are crucial for the solution procedure.

Cuts derived from any trial solution $\hat{x}_{t}^{\omega_{t}}$ are valid in all scenarios $\omega_{t} \in \Omega_{t}$.

The cut $\theta_{t} \geq E_{\omega_{t+1}} \pi_{t+1}^{\omega_{t+1}} B_{t}^{\omega_{t+1}} x_{t}+E_{\omega_{t+1}}\left(\pi_{t+1}^{\omega_{t+1}} b_{t+1}^{\omega_{t+1}}+\sum_{l_{t+1}=1}^{L_{t+1}} \rho_{t+1}^{l_{t+1}, \omega_{t+1}} g_{t+1}^{l_{t+1}}\right)$ is a constraint whose coefficients do not depend on $x_{t}$; hence, it is valid for all values of $x_{t}$. We exhibit this property via the example of cuts generated for the stage $T-1$ master problem and show that these cuts are valid for all values $x_{T-1}^{\omega_{T-1}}$. We employ dual (Benders) decomposition theory to obtain the relation between the stage $T-1$ master problem and the stage $T$ subproblems. First we re-state the stage $T-1$ master problem of the multi-stage stochastic linear program in the following form:

The Stage $T-1$ Relaxed Master Problem:

$$
\begin{aligned}
z_{T-1}=\min c_{T-1} x_{T-1} & +z_{T}\left(x_{T-1}\right) \\
\mathrm{s} / \mathrm{t} \quad\left(A_{T-1} x_{T-1}\right. & \\
x_{T-1} & \left.\geq b_{T-1}^{\omega_{T-1}}+B_{T-2}^{\omega_{T-1}} \hat{x}_{T-2}\right) \\
& \geq 0 .
\end{aligned}
$$

System (4.19) represents the relaxed stage $T-1$ master problem, where no cuts have been added so far. As an initial step, we also relax the stage $T-1$ original constraints $\left(A_{T-1} x_{T-1}=b_{T-1}^{\omega_{T-1}}+B_{T-2}^{\omega_{T-1}} \hat{x}_{T-2}\right)$, as indicated by setting them between prientheses. This is to make the stage $T-1$ problem independent of the different scenarios $\omega_{T-1}$ and independent of any history that is represented by $\hat{x}_{T-2}$ passed from the previous stage. Thus we can interpret the stage $T-1$ master problem as being completely cut off from the previous stages. The expected (future) stage $T$ costs, $z_{T}\left(x_{T-1}\right)$, are represented as a function of the stage $T-1$ decision variable $x_{T-1}$.

Instead of stating the primal stage $T$ problem, we state its dual problem. The dual stage $T$ problem in (4.20) includes all $K_{T}$ scenarios $\omega_{T} \in \Omega_{T}$. Note that the stage $T-1$ decision, $\hat{x}_{T-1}$, appears as a given parameter of the stage $T$ subproblem. 


\section{The Stage $T$ Dual Subproblem:}

$$
\begin{aligned}
& z_{T}\left(\hat{x}_{T-1}\right)= \\
& \max p_{T}^{1} \pi_{T}^{1}\left(b_{T}^{1}+B_{T-1}^{1} \hat{x}_{T-1}\right)+\cdots+p_{T}^{K_{T}} \pi_{T}^{K_{T}}\left(b_{T}^{K_{T}}+B_{T-1}^{K_{T}} \hat{x}_{T-1}\right) \\
& \mathrm{s} / \mathrm{t}
\end{aligned}
$$

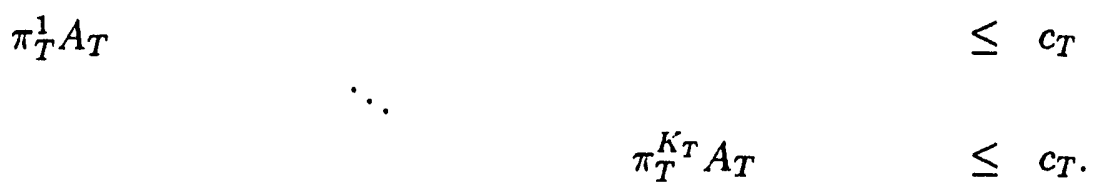

We assume the stage $T$ dual subproblem to be finite for all values of $\hat{x}_{T-1}$ passed from the previous period. We define

$$
\pi_{T}^{j}:=\left(\pi_{T}^{1, j}, \ldots, \pi_{T}^{K_{T}, j}\right), j=1, \ldots, q_{T}
$$

to be the vertices of the dual feasible region of the stage $T$ subproblem (4.20). We rewrite the stage $T$ subproblem (4.20) by expressing it in terms of the dual vertices. By doing so we also can write the maximum expected costs $z_{T}\left(x_{T-1}\right)$ as a function of $x_{T-1}$, rather than having the problem parameterized by $\hat{x}_{T-1}$ :

$$
z_{T}\left(x_{T-1}\right)=\max _{1 \leq j \leq q T} p_{T}^{1} \pi_{T}^{1, j}\left(b_{T}^{1}+B_{T-1}^{1} x_{T-1}\right)+\cdots+p_{T}^{K_{T}} \pi_{T}^{K_{T}, j}\left(b_{T}^{K_{T}}+B_{T-1}^{K_{T}} x_{T-1}\right) .
$$

Using $\theta_{T-1}$ to represent the smallest upper bound for the stage $T$ costs,

$$
\begin{aligned}
& \min \theta_{T-1} \\
& \theta_{T-1} \geq \max _{1 \leq j \leq q T} \quad p_{T}^{1} \pi_{T}^{1, j}\left(b_{T}^{1}+B_{T-1}^{1} x_{T-1}\right)+\cdots+p_{T}^{K_{T}} \pi_{T}^{K_{T}, j}\left(b_{T}^{K_{T}}+B_{T-1}^{K_{T}} x_{T-1}\right),
\end{aligned}
$$

we can state the stage $T-1$ master problem as a full master problem with all possible cuts added.

The Stage $T-1$ Full Master Problem:

$$
\begin{aligned}
& \min z_{T-1}= c_{T-1} x_{T-1}+\theta_{T-1} \\
& \mathrm{~s} / \mathrm{t}\left.=b_{T-1}^{A_{T-1}}+x_{T-1}^{\omega_{T-1}}+B_{T-1}^{\omega_{T-1}} \hat{x}_{T-2}\right) \\
& x_{T-1} \geq 0 \\
& \theta_{T-1} \geq p_{T}^{1} \pi_{T}^{1, j}\left(b_{T}^{1}+B_{T-1}^{1} x_{T-1}\right)+\cdots+p_{T}^{K_{T}} \pi_{T}^{K_{T}, j}\left(b_{T}^{K_{T}}+B_{T-1}^{K_{T}} x_{T-1}\right) \quad j=1, \ldots, q_{T} .
\end{aligned}
$$

In the full master representation for stage $T-1$, each cut corresponds to a vertex of the dual feasible region of the stage $T$ subproblem. The set of all cuts represents a complete outer linearization of the expected stage $T$ costs as a function of $x_{T-1}$. Each cut is valid for each value of $x_{T-1}$ because it is derived from a dual feasible price vector, one of the dual vertices $\pi_{T}^{j}, j=1, \ldots, q_{T}$. Taking now the stage $T-1$ constraints $\left(A_{T-1} x_{T-1}=b_{T-1}^{\omega_{T-1}}+B_{T-2}^{\omega_{T-1}} \hat{x}_{T-2}\right)$ into consideration, we see that the piecewise-linear representation of the expected stage $T$ costs remains unaffected by 
the stage $T-1$ constraints. The stage $T-1$ constraints have different right-hand sides in each scenario $\omega_{T-1}$ and for each value of $\hat{x}_{T-2}$ passed from the previous period. Therefore they represent a different set of feasible solutions for $x_{T-1}$ in each scenario $\omega_{T-1}$ and for each value of $\hat{x}_{T-2}$. The piecewise-linear representation of the stage $T$ expected costs by means of the cuts is valid for any scenario $\omega_{T-1}$ and any value of $\hat{x}_{T-2}$ passed from the previous period. Of course, different cuts will be binding in different scenarios $\omega_{T-1}$. For example, a cut can be a support of the recourse function, the minimum expected costs $z_{T}\left(x_{T-1}\right)$ as a function of the stage $T-1$ decision variables $x_{T-1}$, in a particular scenario and may be way below the recourse function in another scenario.

Having stated the full master problem in stage $T-1$ in equation (4.24-4.4.1), we now extend the argument for all stages. Analogously to before, we state the dual problems in stages $t$, where $t=2, \ldots, T-1$. We assume that the subproblem in stage $t$ under consideration contains a complete representation of the stage $t+1$ expected costs, represented by the set of cuts $-G_{t}^{l_{t}} x_{t}^{\omega_{t}}+\theta_{t}^{\omega_{t}} \geq g_{t}^{l_{t}}, l_{t}=1, \ldots, q_{t}$, and that these cuts also have been derived from subproblems with a full representation of their respective future expected costs.

The Stage $t, t=2, \ldots, T-1$ Dual Subproblem:

$z_{t}\left(\hat{x}_{t-1}\right)=$

$$
\begin{aligned}
& \max p_{t}^{1}\left(\pi_{t}^{1}\left(b_{t}^{1}+B_{t-1}^{1} \hat{x}_{t-1}\right)+\rho_{t}^{1} g_{t}\right)+\cdots+p_{T}^{K_{t}}\left(\pi_{t}^{K_{t}}\left(b_{t}^{K_{t}}+B_{t-1}^{K_{t}} \hat{x}_{t-1}\right)+\rho_{t}^{K_{t}} g_{t}\right) \\
& s / t \\
& \pi_{t}^{1} A_{t} \\
& -\rho_{t}^{1} G_{t} \\
& \leq c_{t} \\
& \begin{array}{lll} 
& & \\
\rho_{t}^{1} 1 & & \pi_{t}^{K_{t}} A_{t} \\
& \ddots &
\end{array} \\
& \rho_{t}^{1}, \quad \ldots, \\
& \begin{array}{c}
\rho_{t}^{K_{t}} 1 \\
\rho_{t}^{K_{t}} \geq 1 \\
\geq 0 .
\end{array}
\end{aligned}
$$

As before, the stage $t-1$ master problem in the full master representation can be stated as:

The Stage $t-1$ Full Master Problem:

$$
\begin{array}{rlrl}
\min z_{t-1} & = & c_{t-1} x_{t-1} \\
\mathrm{~s} / \mathrm{t} & +\theta_{t-1} x_{t-1} & & \\
& & \left.\geq b_{t-1}^{\omega_{t-1}}+B_{t-2}^{\omega_{t-1}} \hat{x}_{t-2}\right) \\
& & \geq 0
\end{array}
$$




$$
\begin{aligned}
& \theta_{t-1} \geq \\
& p_{t}^{1}\left(\pi_{t}^{1, j}\left(b_{t}^{1}+B_{t-1}^{1} x_{t-1}\right)+\rho_{t}^{1, j} g_{t}\right)+\cdots+\left(p_{t}^{K_{t}} \pi_{t}^{K_{t}, j}\left(b_{t}^{K_{t}}+B_{t-1}^{K_{t}} x_{t-1}\right)+\rho_{t}^{K_{t}, j} g_{t}\right), \\
& j=1, \ldots, q_{t} .
\end{aligned}
$$

Equation (4.28) represents a complete outer-linearization of the future costs as a function of $x_{t-1}$. It is valid for all possible values of $x_{t-1}$. The stage $t-1$ constraints have different right-hand sides for different scenarios $\omega_{t-1}$ and for different values of $\hat{x}_{t-2}$ passed from the previous stage. They represent a different set of feasible solutions of $x_{t-1}$ in each scenario $\omega_{t-1}$ and for each value of $\hat{x}_{t-2}$, but the outer linearization of the stage $t+1$ costs is valid in any scenario $\omega_{t-1}$ and for each value of $\hat{x}_{t-2}$ passed from the previous period. Therefore all cuts $-G_{t-1}^{l_{t-1}} x_{t-1}^{\omega_{t-1}}+\theta_{t-1}^{\omega_{t-1}} \geq g_{t-1}^{l_{t-1}}, l_{t-1}=1, \ldots, L_{t-1}$ are valid for all scenarios $\omega_{t-1} \in \Omega_{t-1}$.

\section{A cut derived from a non-extreme dual feasible point is a valid cut.}

In the full master representation of the stage $t-1$ problem in (4.27) and (4.28) each cut has been derived from a vertex of the dual feasible region of the stage $t$ subproblem. A cut derived from a dual extreme point is efficient in the sense that it supports the expected future cost function $z_{t}\left(x_{t-1}\right)$ at that value of $\hat{x}_{t-1}$ for which the dual extreme point is optimal. Of course, not every dual extreme point need be optimal for some value of $x_{t-1}$. A cut derived from a point within the dual feasible region, which is not an extreme point, cannot be a support of $z_{t}\left(x_{t-1}\right)$ for any value of $x_{t-1}$, because a point within the dual feasible region, which is not an extreme point, is never dual optimal. Therefore, it is not efficient in the above sense.

For example, any point of the feasible region of the stage $t$ dual subproblem of (4.26) can be represented by a linear combination of the extreme poinis, e.g.,

$$
\left(\pi_{t}^{0}, \rho_{t}^{0}\right)=\sum_{l_{t}=1}^{q_{t}} \lambda^{l_{t}}\left(\pi_{t}^{l_{t}}, \rho_{t}^{l_{t}}\right), \quad \sum_{l_{t}=1}^{q_{t}} \lambda^{l_{t}}=1, \quad \lambda^{l_{t}} \geq 0 .
$$

For any value of $x_{t}-1$ the outer linearization of the expected stage $t$ costs, based on the dual interior point $\pi_{t}^{0}, \rho_{t}^{0}$,

$$
z_{t}^{0}\left(x_{t-1}\right)=E_{\omega_{\mathrm{t}}}\left(\pi_{t}^{\omega_{t}, 0}\left(b_{t}^{\omega_{t}}+B_{t-1}^{\omega_{t}} x_{t-1}\right)+\rho_{t}^{\omega_{t}, 0} g_{t}\right)
$$

is less than the outer linearization corresponding to the extreme point $\pi_{t}^{\max }, \rho_{t}^{\max }$ which maximizes the dual problem for a particular $\hat{x}_{t-1}$ :

$$
z_{t}^{0}\left(x_{t-1}\right) \leq E_{\omega_{t}}\left(\pi_{t}^{\omega_{t}, \max }\left(b_{t}^{\omega_{t}}+B_{t-1}^{\omega_{t}} x_{t-1}\right)+\rho_{t}^{\omega_{t}, \max } g_{t}\right),
$$

where

$$
E_{\omega_{t}}\left(\pi_{t}^{\omega_{t}, \max }\left(b_{t}^{\omega_{t}}+B_{t-1}^{\omega_{t}} x_{t-1}\right)+\rho_{t}^{\omega_{t}, \max } g_{t}\right)=\max _{j} E_{\omega_{t}}\left(\pi_{t}^{\omega_{t}, j}\left(b_{t}^{\omega_{t}}+B_{t-1}^{\omega_{t}} x_{t-1}\right)+\rho_{t}^{\omega_{t}, j} g_{t}\right) .
$$

It follows that a cut obtained from a dual feasible point that is not an extreme point of the dual feasible region in (4.26) is weak, but valid for any value of $x_{t-1}$ of the stage $t-1$ master problem. 
An extreme point of the dual feasible region of a subproblem that is not balanced with its decendent stage is also an extreme point of its dual feasible region when it is balanced with its descendent stage.

We analyse now the case where the stage $t$ subproblem is not fully balanced with the stage $t+1$ subproblem, that is, when not all cuts have been generated and added to the stage $t$ subproblem to fully represent the expected stage $t+1$ costs, $z_{t+1}\left(x_{t}\right)$, given $\hat{x}_{t-1}$ passed to the stage $t$ problem and for all scenarios $\omega_{t} \in \Omega_{t}$ in stage $t$. To determine, if the cuts added so far to the stage $t$ subproblem fully represent the stage $t+1$ expected costs, we define $\theta^{\min }\left(x_{t}\right)$ as the smallest upper bound of the value of the stage $t+1$ costs by means of the stage $t$ cuts in the stage $t$ subproblem:

$$
\theta^{\min }\left(x_{t}\right)=\min _{l_{t}} \theta_{t}, \theta_{t} \geq G_{t}^{l_{t}} x_{t}+g_{t}^{l_{t}}, l_{t}=1, \ldots, L_{t} .
$$

If

$$
\theta^{\min }\left(x_{t}\right)=z_{t+1}\left(x_{t}\right)
$$

then the cuts added so far in stage $t$ fully represent the stage $t+1$ expected costs, and the stage $t$ subproblem is balanced with the stage $t+1$ subproblem. Otherwise, additional cuts have to be generated for stage $t$ in order to obtain a complete representation of the stage $t+1$ expected costs.

The case of the stage $t$ subproblem not being balanced with the stage $t+1$ subproblem can be interpreted in these terms: the dimension of the vector of dual prices $\rho_{t}$, corresponding to the number of cuts added so far to the stage $t$ subproblem, is smaller than its dimension would be if all cuts were added to fully represent the expected future costs $z_{t+1}\left(x_{t}\right)$. For example, the stage $t$ full master problem had $q_{t}$ cuts but only $L_{t}$ cuts have been added so far. The stage $t$ subproblem then can be seen as having $q_{t}-L_{t}$ relaxed constraints, whose coefficients and right-hand sides are zero. The dual variables associated with these relaxed constraints are defined to have the value zero. In the dual representation of the stage $t$ subproblem these relaxed rows appear as zero-columns. An extreme point $\pi_{t}^{j}, \rho_{t}^{j}$ of the dual feasible region corresponding to $L_{t}$ cuts and with $q_{t}-L_{t}$ zero-columns is also an extreme point of the dual feasible region corresponding to $q_{t}$ cuts and for which $\rho_{t}^{j} \geq 0$, for $j=L_{t}+1, \ldots, q_{t}$. This is because if $\left(\pi_{t}, \rho_{t}^{1}, \ldots, \rho_{t}^{L_{t}}\right)$, is an extreme point of the relaxed dual, then the constructed dual solution $\left(\pi_{t}, \rho_{t}^{1}, \ldots, \rho_{t}^{L_{t}}, \rho_{t}^{L_{t}+1}=0, \ldots, \rho_{t}^{q_{t}}=0\right)$ could not be formed from a linear combination of other extreme points of the full system by vertue of the non-negativity of $\rho_{t}$. A cut obtained from a dual extreme point is efficient in the sense that it has the potential to be support of the recourse function for some value of $x_{t}$. Therefore a cist obtained from stage $t+1$ subproblems, which are not balanced with its descendent subproblems in stage $t+2$ is an efficient cut. It is suboptimal for this particular value of $\hat{x}_{t}$ at which it was derived but it may be optimal for another value of $x_{t}$. Note that this result has an important implication: No matter at which stage cuts are computed, regardless of the corresponding subproblems being balanced or unbalanced with their decendent stage, the obtained cuts are always efficient cuts. 
They are potentially support of the recourse function at some value of $x_{t}$. Note that cuts computed in the first stage are efficient cuts, even if no cuts have been added in stages $2, \ldots, T-1$, that is, if no information about the future is collected.

\section{Cuts obtained from expected value subproblems are valid cuts.}

By replacing all stochastic parameters $B_{t-1}^{\omega_{t}}, b_{t}^{\omega_{t}}, t=2, \ldots, T$ by their expected values $B_{t-1}^{E}=E_{\omega_{t}}\left(B_{t-1}^{\omega_{t}}\right), b_{t}^{E}=E_{\omega_{t}}\left(b_{t}^{\omega_{t}}\right)$, we obtain from the multi-stage stochastic linear program of (4.2) a deterministic multi-stage linear program (4.35), which we refer to as its expected value problem.

The expected value problem:

$$
\begin{aligned}
& \min z= \\
& c_{1} x_{1}+c_{2} x_{2}+\cdots+c_{T-1} x_{T-1}+c_{T} x_{T} \\
& \mathrm{~s} / \mathrm{t} \\
& A_{1} x_{1} \\
& -B_{1}^{E} x_{1}+A_{2} x_{2} \\
& =b_{1} \\
& =b_{2}^{E} \\
& \begin{array}{rlrrr} 
& & -B_{T-1}^{E} x_{T-1},+ & A_{T} x_{T} & =b_{T}^{E} \\
x_{1}, \quad x_{2}, \ldots, & x_{T-1}, & x_{T} & \geq 0 .
\end{array}
\end{aligned}
$$

In order to demonstrate the properties of cuts obtained from expected value subproblems, we apply dual (Benders) decomposition to (4.35). Corresponding to the stage $t$ subproblem (4.13) of the multi-stage stochastic linear problem is the stage $t$ subproblem (4.36) of the decomposed multi-stage expected value problem:

The stage $t$ expected value subproblem:

$$
\begin{aligned}
& \min z_{t}^{E}\left(\hat{x}_{t-1}\right)=\min c_{t} x_{t}+\theta_{t} \\
& \begin{array}{rlrl}
\mathrm{s} / \mathrm{t} & & \\
\pi_{t}^{E}: & A_{t} x_{t} & =b_{t}^{E}+B_{t-1}^{E} \hat{x}_{t-1} \\
\rho_{t}^{E}: & -G_{t}^{E} x_{t}+\theta_{t} & \geq g_{t}^{E} \\
x_{t} & \geq 0 .
\end{array}
\end{aligned}
$$

In the stochastic case we have defined the expected stage $t$ costs as a function of $x_{t-1}$ as

$$
z_{t}\left(x_{t-1}\right)=E_{\omega_{t}}\left(z_{t}^{\omega_{t}}\left(x_{t-1}\right)\right),
$$

where $z_{t}^{\omega_{t}}\left(x_{t-1}\right)$ is a convex function. From this it follows (based on Jensen's inequality for convex functions) that

$$
z_{t}\left(x_{t-1}\right) \geq z_{t}^{E}\left(x_{t-1}\right) .
$$

Equation (4.38) is true for all values of $x_{t-1}$. 
By the usual argument for Benders decomoposition, a cut derived from the expected value subproblem is an outer linearization of the stage $t$ expected value subproblem costs $z_{t}^{E}\left(x_{t-1}\right)$. To show this we assume that problem (4.36) is feasible for all values of $\hat{x}_{t-1}$ and define $\pi_{t}^{E, j}, \rho_{t}^{E, j}, j=1, \ldots, q_{t}^{E}$ to be the extreme points of its dual feasible region, where $q_{t}^{E}$ denotes the number of dual vertices. The outer linearization then is represented as

$$
L_{t}^{E}\left(x_{t-1}\right)=\max _{j} \pi_{t}^{E, j}\left(b_{t}^{E}+B_{t-1}^{E} x_{t-1}\right)+\rho_{t}^{E, j} g_{t}, \quad j=1, \ldots, q_{t}^{E} .
$$

With

$$
z_{t}^{E}\left(x_{t-1}\right) \geq L_{t}^{E}\left(x_{t-1}\right)
$$

we can state

$$
z_{t}\left(x_{t-1}\right) \geq L_{t}^{E}\left(x_{t-1}\right)
$$

This is a valid statement for all values of $x_{t-1}$ and is unaffected by any additional constraints on $x_{t-1}$ in the stage $t-1$ subproblem. It follows from (4.38), then, that cuts obtained from the stage $t$ expected value subproblem are valid but weak cuts for any stage $t-1, \omega_{t-1}$ subproblem of the decomposed stochastic linear problem. Indeed, they are used to guide our solution algorithm at the beginning. For example, we first solve the expected value problem (4.35) using nested dual decomposition and collect cuts at each stage $t, t=1, \ldots, T-1$. Then we solve the stochastic problem, where the expected value cuts are initially present but are gradually replaced by stronger stochastic cuts.

\section{Summary of properties of cuts}

Different scenarios $\omega_{t}$ in stage $t$ are distinguished by different right-hand sides of the original stage $t$ constraints, e.g., $A_{t} x_{t}=b_{t}^{\omega_{t}}+B_{t-1}^{\omega_{t}} \hat{x}_{t-1}$. The set of cuts $-G_{t}^{l_{t}} x_{t}+\theta_{t} \geq$ $g_{t}^{l_{t}}, l_{t}=1, \ldots, L_{t}$, represents an outer linearization of the expected future costs that is independent of stage $t$ scenarios and is valid for all scenarios $\omega_{t} \in \Omega_{t}$. The outer linearization defined by the set of cuts equals the expected future cost function if $E z_{t+1}^{\omega_{t+1}}\left(\hat{x}_{t}\right)=\hat{\theta}_{t}$, where $\hat{\theta}_{t}$ is the value of $\theta_{t}$ corresponding to the solution $\hat{x}_{t}$ of any stage $t$ problem. If $E z_{t+1}^{\omega_{t+1}}\left(\hat{x}_{t}^{\omega_{t}}\right)=\hat{\theta}_{t}^{\omega_{t}}$, for all $\omega_{t} \in \Omega_{t}$, then a sufficient number of necessary cuts has been generated to represent the expected future costs for all solutions $\hat{x}_{t}^{\omega_{t}}$ of scenarios $\omega_{t} \in \Omega_{t}$ in stage $t$, and we say that stage $t$ is balanced with stage $t+1$. In any stage $t-1, t=2, \ldots, T-1$, we can obtain cuts from stage $t$ subproblems which are not balanced with their descendent stage $t+1$ subproblems. The resulting cuts are valid, weak at that $x_{t-1}$, at which they are derived, but potentially efficient at some other value of $x_{t-1}$. Cuts obtained $n$ any stage $t-1, t=2, \ldots, T-1$ from expected value subproblems in stage $t$ are valid for any scenario $\omega_{t-1}$. They are weak, but can be used to guide the algorithm at the beginning. 


\subsubsection{The Case of Dependency of Stochastic Parameters Between Stages}

In the case of inter-stage dependency of stochastic parameters the coefficients and the right-hand side of the cuts depend on the scenario history. Simple sharing of cuts between different scenario subproblems $\omega_{t} \in \Omega_{t}$ is no longer possible. However, for line $x$ (Markovian) dependency, cuts can be easily adjusted to fit different scenarios. (See Pereira and Pinto (1989) [103] for a treatment of a class of additively dependent right-hand sides.)

For the auto-correlative dependency model, cuts in any stage $t$ can be adjusted to be valid in any scenario $\omega_{t} \in \Omega_{t}$.

In the auto-correlative model developed in Section 4.2.2, the transition matrix and the right-hand side in stage $t$, based on the scenario history $\omega_{t-1}, \ldots, \omega_{2}$, are expressed as

$$
\begin{aligned}
& B_{t}^{\omega_{t}, \ldots, \omega_{2}}=\operatorname{vec}_{B}^{-1}\left(\beta_{0} v^{\omega_{t}}\right)+\sum_{\tau=1}^{t-2} \operatorname{vec}_{B}^{-1}\left(\beta_{\tau} \operatorname{vec}\left(B_{t-\tau-1}^{\omega_{t}-\tau}, b_{t-\tau}^{\omega_{t}-\tau}\right)\right), \\
& b_{t}^{\omega_{t}, \ldots, \omega_{2}}=\operatorname{vec}_{b}^{-1}\left(\beta_{0} v^{\omega_{t}}\right)+\sum_{\tau=1}^{t-2} \operatorname{vec}_{b}^{-1}\left(\beta_{\tau} \operatorname{vec}\left(B_{t-\tau-1}^{\omega_{t}-\tau}, b_{t-\tau}^{\omega_{t-\tau}}\right)\right),
\end{aligned}
$$

where $\tau$ represents the index of the time lag. We compute a cut from the (dual) stage $t$ problem as:

$$
\theta_{t-1} \geq E_{\omega_{t}}\left(\pi_{t}^{\omega_{t}}\left(b_{t}^{\omega_{t}, \ldots, \omega_{2}}+B_{t-1}^{\omega_{t}, \ldots, \omega_{2}} x_{t-1}\right)+\rho_{t}^{\omega_{t}} g_{t}^{\omega_{t}, \ldots, \omega_{2}}\right) .
$$

Including the inter-stage dependency according to the auto-correlative model by substitution leads to the following formulation:

$$
\begin{aligned}
& \theta_{t-1} \geq \\
& E_{\omega_{t}} \pi_{t}^{\omega_{t}}\left(\operatorname{vec}_{b}^{-1}\left(\tilde{\beta} \beta_{0} V^{\omega_{t}}\right)\right)+E_{\omega_{t}} \pi_{t}^{\omega_{t}}\left(\operatorname{vec}_{B}^{-1}\left(\tilde{\beta} \beta_{0} V^{\omega_{t}}\right) x_{t-1}\right)+ \\
& \left(E_{\omega_{t}} \pi_{t}^{\omega_{t}}\right)\left(\sum_{\tau=1}^{t-2} \operatorname{vec}_{b}^{-1}\left(\beta_{\tau} \operatorname{vec}\left(B_{t-\tau-1}^{\omega_{t}-\tau}, b_{t-\tau}^{\omega_{t-\tau}}\right)\right)\right)+ \\
& \left(E_{\omega_{t}} \pi_{t}^{\omega_{t}}\right)\left(\sum_{\tau=1}^{t-2} \operatorname{vec}_{B}^{-1}\left(\beta_{\tau} \operatorname{vec}\left(B_{t-\tau-1}^{\omega_{t}-\tau}, b_{t-\tau}^{\omega_{t}-\tau}\right)\right) x_{t-1}\right)+ \\
& E_{\omega_{t}}\left(\rho_{t}^{\omega_{t}} g_{t}^{\omega_{t}, \ldots, \omega_{2}}\right) .
\end{aligned}
$$

The obtained cut consists of two parts: The first part reflects the contribution of the outcomes of the stage $t$ independent random vector $v_{t}^{\omega_{t}}$, the second part reflects the contribution of the observed outcomes in stages $2, \ldots, t-1$. Still, a cut based on the auto-correlative dependency model cannot be shared directly between the subproblems in stage $t-1$. This is, because the coefficients and the right-hand side of a cut from stage $t$ depend on $\omega_{t-1}$ and also on the full history of realizations $\omega_{t} 2, \ldots, \omega_{2}$. However, one can see (easily) that the cut can be adjusted to the current scenario $\omega_{t-1}, \ldots, \omega_{2}$ if the expectation $\left(E_{\omega_{t}} \pi_{t}^{\omega_{t}}\right)$ is known. Note the notation 
of $g_{t}^{\omega_{t}, \ldots, \omega_{2}}$ to indicate that the right hand sides of the cuts in stage $t+1$ have a history because of $b_{t+1}^{\omega_{t+1}, \ldots, \omega_{2}}$. However, based on the linear dependency model we can compute the stage $t$ impact analogously to the derivation above.

In order to adjust the cut to a given scenario $\omega_{t-1}$, we only have to specially compute the second and $\omega_{t-1}$-dependent part of the cut formulation. The first part remains the same for each scenario $\omega_{t-1}, \ldots, \omega_{2}$. Note that the computationally expensive expected value computation is required in both parts. As part one remains the same for each scenario $\omega_{t-1}, \ldots, \omega_{2}$, we store the calculation and adjust the cut for a giiven scenario by adding part two using $\left(E_{\omega_{\mathrm{t}}} \pi_{t}^{\omega_{\mathfrak{t}}}\right)$, the expected value of the dual prices already obtained and stored during the computation of part one.

We exhibit this procedure by considering first the simplified example of the autocorrelative model of (4.11) with uncertainty occurring only in the right-hand sides according to a Markovian dependency of a time lag of 1 :

$$
b_{t}^{\omega_{t}}=\beta_{0} \eta_{t}^{\omega_{t}}+\beta_{1} b_{t-1}^{\omega_{t-1}}
$$

where $\eta_{t}^{\omega_{t}}$ represents a vector (of the dimension of $b_{t}$ ) of independent random parameters and $\beta_{0}, \beta_{1}$ are scalars. The corresponding cut,

$$
\theta_{t-1} \geq E_{\omega_{t}} \pi_{t}^{\omega_{t}}\left(\beta_{0} \eta_{t}^{\omega_{t}}+B_{t-1} x_{t-1}\right)+E_{\omega_{t}} \rho_{t}^{\omega_{t}} g_{t}^{\omega_{t}}+\left(E_{\omega_{t}} \pi_{t}^{\omega_{t}}\right) \beta_{1} b_{t-1}^{\omega_{t-1}}
$$

only needs to be adjusted in the right-hand side by specializing the term $\left(E_{\omega_{t}} \pi_{t}^{\omega_{t}}\right) \beta_{1} b_{t-1}^{\omega_{t-1}}$ to the stage $t-1, \omega_{t-1}$ scenario subproblem which we currently want to solve.

Alternatively, consider the simplified example of the auto-correlative model of (4.10) in which uncertainty occurs only in the transition matrix, according to a Markovian dependency with a time lag of 1 :

$$
B_{t-1}^{\omega_{t}}=\beta_{0} \bar{\eta}_{t}^{\omega_{t}}+\beta_{1} B_{t-2}^{\omega_{t-1}}
$$

where $\bar{\eta}_{t}^{\omega_{t}}$ is a matrix (of the dimension of $B_{t-1}$ ) of independent random parameters and $\beta_{0}, \beta_{1}$ are scalars. The corresponding cut,

$$
\theta_{t-1} \geq E_{\omega_{t}} \pi_{t}^{\omega_{t}}\left(b_{t}+\beta_{0} \bar{\eta}_{t} x_{t-1}\right)+E_{\omega_{\imath}} \rho_{t}^{\omega_{t}} g_{t}^{\omega_{t}}+\left(E_{\omega_{t}} \pi_{t}^{\omega_{t}}\right) \beta_{1} B_{t-2}^{\omega_{t}-1} x_{t-1}
$$

only needs to be adjusted in its coefficients by specializing the term $\left(E_{\omega_{t}} \pi_{t}^{\omega_{t}}\right) \beta_{1} B_{t-2}^{\omega_{t}-1}$ to the stage $t-1, \omega_{t-1}$ scenario subproblem which is to be solved.

\subsubsection{Summary}

Taking advantage of the above stated properties, we actually only need to store one subproblem per stage $t$. For different scenarios $\omega_{t}$ and different solutions $\hat{x}_{t-1}$ passed from the previous stage we determine the right-hand side of the subproblem accordingly. Cuts generated by stage $t+1$ subprobiems are valid for all scenarios $\omega_{t} \in \Omega_{t}$ in the case of independence of the stochastic parameters between stages. Generated cuts are adjusted in the gradient and right-hand side according to the 
actual scenario $\omega_{t}$ in the case of Markovian dependency between stages. Therefore it is easy to generate any $\omega_{t}$ subproblem. Future information is represented in the cuts which have been generated so far and can be efficiently used in any scenario $\omega_{t} \in \Omega_{t}$ independently of which scenario originated the cut:

\subsection{PROBABILISTIC LOWER BOUNDS}

\subsubsection{Estimates of Expected Values}

For calculating the expected values of the future costs, the gradients, and the righthand sides of the cuts in each stage $t$, we use Monte Carlo importance sampling as discussed in Section 2.3.3 in Chapter "Benders Decomposition and Importance Sampling for Stochastic Linear Programs" for two-stage stochastic linear programs. Employing Monte Carlo sampling techniques means not solving all problems $\omega_{t+1} \epsilon$ $\Omega_{t+1}$ but solving problems $\omega_{t+1} \in S_{t+1}$, where $S_{t+1}$ is a subset of $\Omega_{t+1}$. Instead of the exact expected values $z_{t+1}\left(\hat{x}_{t}\right), G_{t}\left(\hat{x}_{t}\right), g_{t}\left(\hat{x}_{t}\right)$, we compute estimates $\bar{z}_{t+1}\left(\hat{x}_{t}\right), \bar{G}_{t}\left(\hat{x}_{t}\right)$, $\bar{g}_{t}\left(\hat{x}_{t}\right)$ using the importance sampling procedure. We also estimate the error in the estimation of $z_{t+1}\left(\hat{x}_{t}\right)$ by the variance

$$
\sigma_{\bar{z}_{t+1}}^{2}\left(\hat{x}_{t}\right):=\operatorname{var}\left(\bar{z}_{t+1}\left(\hat{x}_{t}\right)\right) .
$$

Thus, given a particular $\hat{x}_{t}$, we obtain from the importance sampling procedure an estimate of the mean of the stage $t+1$ costs and the associated error distribution. For sample sizes larger than 40 (see, for example, Davis and Rabinowitz (1984) [32]), one can assume that the error of the estimation is normally distributed. Therefore, we define the estimate of the stage $t+1 \operatorname{costs} \tilde{z}_{t+1}\left(\hat{x}_{t}\right)$ for given $\hat{x}_{t}$ to be a random parameter, normally distributed with mean $\bar{z}_{t+1}\left(\hat{x}_{t}\right)$ and variance $\sigma_{\bar{z}_{t+1}}^{2}$ :

$$
\tilde{z}_{t+1}\left(\hat{x}_{t}\right):=N\left(\bar{z}_{t+1}\left(\hat{x}_{t}\right), \sigma_{\bar{z}_{t+1}}^{2}\left(\hat{x}_{t}\right)\right) .
$$

A cut with estimated coefficients and right-hand sides differs from a cut obtained by computing the expected values of the coefficients and right-hand sides exactly. The outer linearization

$$
L_{t}\left(\hat{x}_{t}, x_{t}\right)=G_{t}\left(\hat{x}_{t}\right) x_{t}+g_{t}\left(\hat{x}_{t}\right)
$$

with respect to the universe case and

$$
\bar{L}_{t}\left(\hat{x}_{t}, x_{t}\right)=\bar{G}_{t}\left(\hat{x}_{t}\right) x_{t}+\bar{g}_{t}\left(\hat{x}_{t}\right)
$$

with respect to the estimation differ in the gradient and in the right-hand side. At $x_{t}=\hat{x}_{t}$, the value at which the cut was derived, $L_{t}\left(\hat{x}_{t}, \hat{x}_{t}\right)=z_{t+1}\left(\hat{x}_{t}\right)$ and $\bar{L}_{t}\left(\hat{x}_{t}, \hat{x}_{t}\right)=$ $\bar{z}_{t+1}\left(\hat{x}_{t}\right)$. Thus, if a true cut obtained by solving the universe case is binding at the solution $x_{t}=\hat{x}_{t}$, the variable $\theta_{t}$ takes on the value

$$
\theta_{t}=L_{t}\left(\hat{x}_{t}, \hat{x}_{t}\right)=z_{t+1}\left(\hat{x}_{t}\right) .
$$


In the case of using Monte Carlo sampling, we relate $\theta_{t}$ to the estimated value of the stage $t+1$ costs at $\hat{x}_{t}, \bar{L}_{t}\left(\hat{x}_{t}, \hat{x}_{t}\right)=\bar{z}_{t+1}\left(\hat{x}_{t}\right)$, and correct for estimation error by adjusting the right-hand side. Thus we can state

$$
\begin{gathered}
\theta_{t}=\bar{L}_{t}\left(\hat{x}_{t}, \hat{x}_{t}\right)-\bar{z}_{t+1}\left(\hat{x}_{t}\right)+z_{t+1}\left(\hat{x}_{t}\right), \\
\theta_{t}=\bar{L}_{t}\left(\hat{x}_{t}, \hat{x}_{t}\right)+\epsilon_{t}\left(\hat{x}_{t}\right) .
\end{gathered}
$$

Equation (4.55) represents a valid statement for a solution $x_{t}=\hat{x}_{t}$. The correction term $\epsilon_{t}\left(\hat{x}_{t}\right)=z_{t+1}\left(\hat{x}_{t}\right)-\bar{z}_{t+1}\left(\hat{x}_{t}\right)$ corrects for the estimation error. Of course, we do not know the difference $z_{t+1}\left(\hat{x}_{t}\right)-\bar{z}_{t+1}\left(\hat{x}_{t}\right)$ explicitly for each cut when we compute it. However, we can obtain an estimate of the distribution of the correction term from the estimation process. Recall that by using Monte Carlo importance sampling, we obtain an unbiased estimate of $z_{t+1}\left(\hat{x}_{t}\right), \bar{z}_{t+1}\left(\hat{x}_{t}\right)$, with variance $\sigma_{\bar{t}_{t+1}}^{2}\left(\hat{x}_{t}\right)$. Therefore, $\epsilon_{t}\left(\hat{x}_{t}\right)$ is normally distributed with mean 0 and variance $\left.\sigma_{\bar{z}_{t+1}}^{2}\left(\hat{x}_{t}\right)\right)$ :

$$
\epsilon_{t}\left(\hat{x}_{t}\right):=N\left(0, \sigma_{\bar{z}_{t+1}}^{2}\left(\hat{x}_{t}\right)\right) \text {. }
$$

Suppose that a cut $\bar{L}_{t}\left(\hat{x}_{t}, x_{t}\right)=\bar{G}_{t}(\hat{x}) x+\bar{g}(\hat{x})$, computed at $x_{t}=\hat{x}_{t}$, is binding at a solution $\hat{\hat{x}}_{t}$ where $\hat{x}_{t} \neq \hat{x}_{t}$. Applying again a correction for the estimation error we obtain:

$$
\theta_{t}=\bar{L}_{t}\left(\hat{x}_{t}, \hat{\hat{x}}_{t}\right)+\epsilon_{t}\left(\hat{\hat{x}}_{t}\right) \text {. }
$$

The correction term for the estimation error is clearly the true value of the cut at $x_{t}=\hat{\hat{x}}_{t}$, minus the value obtained by the sampling procedure:

$$
\epsilon_{t}(\hat{\hat{x}})=L_{t}\left(\hat{x}_{t}, \hat{\hat{x}}\right)-\bar{L}_{t}\left(\hat{x}_{t}, \hat{\hat{x}}\right) .
$$

Again we do not know the difference $L_{t}\left(\hat{x}_{t}, \hat{\hat{x}}\right)-\bar{L}_{t}\left(\hat{x}_{t}, \hat{\hat{x}}\right)$ when we compute the cut. The distribution of the estimation error, $\epsilon_{t}(\hat{\hat{x}})$ at $x_{t}=\hat{\hat{x}}_{t}$, can be computed based on the observations $G_{t}^{\omega}$ and $g_{t}^{\omega}$, which have been obtained by the estimation procedure. See Section 2.4.2 in Chapter "Benders Decomposition and Importance Sampling for Stochastic Linear Programs" for computing the estimation error of the value of a cut as a function of $x$.

For most practical problems it is a sufficiently close approximation to assume that

$$
\epsilon_{t}\left(\hat{\hat{x}}_{t}\right) \approx \epsilon_{t}\left(\hat{x}_{t}\right) \text { for } \hat{\hat{x}}_{t} \approx \hat{x}_{t} \text {. }
$$

This means that we assume the error distribution $\epsilon_{t}\left(\hat{x}_{t}\right)$ to be constant with respect to $x_{t}$, rather than taking into account correctly $\epsilon_{t}\left(x_{t}\right)$ as a function of $x_{t}$.

\subsubsection{The Lower Bound Estimate}

In each stage $t, t=2, \ldots, T-1$ a lower bound for the stage $t$ expected costs (subject to scenario $\omega_{t}$ and the solution $\hat{x}_{t-1}$ passed from the previous stage) is represented by the optimal objective function value of the stage $t$ master problem, $z_{t}^{\omega_{t}}$. In each 
stage $t, t=2, \ldots, T-1$, the cuts added so far represent an outer linearization of the expected future costs. For an optimal solution $\left(\hat{x}_{t}, \hat{\theta}_{t}\right)$ of the stage $t$ master problem, $\hat{\theta}_{t}$ represents a lower bound for the value of the expected future costs. A lower bound for the total expected costs of the multi-stage stochastic linear program is represented by the optimal objective function value of the stage 1 master problem, $z_{1}$, which includes a value for $\hat{\theta}_{1}$ representing a lower bound for the value of the expected future costs (in stages $2, \ldots, T$ ). If cuts have been obtained in stage $t$ by solving the stage $t+1, \omega_{t+1}$ subproblems, where stage $t+1$ is not balanced with stage $t+2$ (i.e. the stage $t+1$ cuts do not fully represent the stage $t+2$ expected costs), then the stage $t$ cuts are potentially weak cuts. They are not support of the recourse function at that $x_{t}$, from which they were derived, but they have the potential to be support of the recourse function at some other $x_{t}$. This situation is the case, if in stage $t+1$, for a solution $\hat{x}_{t+1}^{\omega_{t+1}}, \hat{\theta}_{t+1}^{\omega_{t+1}}$

$$
\hat{\theta}_{t+1}^{\omega_{t+1}} \leq z_{t+2}\left(\hat{x}_{t+1}^{\omega_{t+1}}\right) .
$$

The corresponding lower bound is then a weak lower bound.

If the cuts are obtained by Monte Carlo sampling rather than by solving the universe case, the optimal objective function value of the stage $t, \omega_{t}$ problem, $z_{t}^{\omega_{t}}$, represents an estimated lower bound for the stage $t, \omega_{t}$ expected costs (subject to $\hat{x}_{t-1}$ passed from the previous stage). The optimal objective function value of the stage 1 problem, $z_{1}$, represents an estimate for the lower bound of the expected costs of the multi-stage stochastic linear program.

In the following we derive a lower bound estimate for the multi-stage stochastic linear problem by analyzing the decomposed program, where at stages $t=1, \ldots, T-1$, $L_{t}$ cuts estimated by Monte Carlo (importance) sampling have been added. We start with an analysis of the relationship between stage $T-1$ and stage $T$.

The Stage $T-1$ Master Problem:

$$
\begin{array}{rrl}
\tilde{z}_{T-1}^{\omega_{T-1}}=\min c_{T-1} x_{T-1}^{\omega_{T-1}}+\theta_{T-1}^{\omega_{T-1}} & \\
\mathrm{~s} / \mathrm{t} & A_{T-1} x_{T-1}^{\omega_{T-1}} & =b_{T-1}^{\omega_{T-1}}+B_{t-2}^{\omega_{T-1}} \hat{x}_{t} \\
\pi_{T-1}^{\omega_{T-1}}: & \bar{G}_{T-1}^{1} x_{T-1}^{\omega_{T-1}}+\theta_{T-1}^{\omega_{T-1}} & \geq \bar{g}_{T-1}^{1}+\epsilon_{T-1}^{1} \\
\rho_{T-1}^{1, \omega_{T-1}}: & \vdots \\
\vdots & & \vdots \\
\rho_{T-1}^{L_{T-1}, \omega_{T-1}}: & -\bar{G}_{T-1}^{L_{T-1}} x_{T-1}^{\omega_{T-1}}+\theta_{T-1}^{\omega_{T-1}} & \geq \bar{g}_{T-1}^{L_{T-1}}+\epsilon_{T-1}^{L_{T-1}} \\
& x_{T-1}^{\omega_{T-1}} &
\end{array}
$$

The Stage $T$ Subproblem:

$$
\begin{aligned}
\min z_{T}^{\omega_{T}} & =c_{T} x_{T}^{\omega_{T}} \\
\mathrm{~s} / \mathrm{t} & \\
\pi_{T}^{\omega_{T}}: & A_{T} x_{T}^{\omega_{T}}=b_{T}^{\omega_{T}}+B_{T-1}^{\omega_{T}} \hat{x}_{T-1} \\
& x_{T}^{\omega_{T}} \geq 0 .
\end{aligned}
$$


For a given scenario $\omega_{T-1}$ and a given solution $\hat{x}_{T-2}$ passed from the stage $T-1$ problem, the relation between stage $T-1$ and stage $T$ corresponds to that of a twostage stochastic linear program. We therefore can apply the theory which we have developed for solving two-stage stochastic linear programs directly.

In the stage $T-1$ problem, $L_{T-1}$ cuts have been added so far. These cuts have been obtained by passing solutions $\hat{x}_{T-1}^{l_{T-1}}, l_{T-1}=1, \ldots, L_{T-1}$, to stage $T$ and solving a sample set $S_{T}$ of stage $T$ subproblems according to the importance sampling scheme. By doing so we have obtained estimates of the stage $T$ expected costs $z_{T}\left(\hat{x}_{T-1}^{T_{T-1}^{-1}}\right)$ and of the gradients $G_{T-1}^{l_{T-1}}$ and right-hand sides $g_{T-1}^{l_{T-1}}$ of the cuts. These estimates are denoted by $\bar{z}_{T}\left(\hat{x}_{T-1}^{l_{T-1}}\right), \bar{G}_{T-1}^{l_{T-1}}$ and $\bar{g}_{T-1}^{l_{T-1}}$. We also have obtained the error correction terms $\epsilon_{T-1}^{l_{T-1}}\left(x_{T-1}\right)$ based on the estimation error at $\hat{x}_{T-1}^{l_{T-1}}$, which for ease of exposition we assume to be constant with respect to $x_{t}$. In general their distribution vary with respect to $x_{T-1}$; see Section 2.4.2 in Chapter "Benders Decomposition and Importance Sampling for Stochastic Linear Programs". However, assuming the error as constant with respect to $x_{T-1}$, each error term $\epsilon_{T-1}^{l_{T-1}}$ then arises from a normal distribution with mean 0 and variance $\operatorname{var}\left(\bar{z}_{T}\left(\hat{x}_{T-1}^{l_{T-1}}\right)\right)$. We denote

$$
\left(\sigma_{T-1}^{l_{T-1}}\right)^{2}:=\operatorname{var}\left(\bar{z}_{T}\left(\hat{x}_{T-1}^{l_{T-1}}\right)\right)
$$

Thus,

$$
\epsilon_{T-1}^{l_{T-1}}=N\left(0,\left(\sigma_{T-1}^{l_{T-1}}\right)^{2}\right)
$$

Based on a local error analysis of the stage $T-1$ problem (4.62) in scenario $\omega_{T-1}$ given $\hat{x}_{T-2}$, the optimal objective function value $\tilde{z}_{T-1}^{\omega_{T-1}}$ is a random parameter, norrnally distributed with mean $\bar{z}_{T-1}^{\omega_{T-1}}$ and variance $\operatorname{var}\left(\bar{z}_{T-1}^{\omega_{T-1}}\right)$,

$$
\tilde{z}_{T-1}^{\omega_{T-1}}=N\left(\bar{z}_{T-1}^{\omega_{T-1}}, \operatorname{var}\left(\bar{z}_{T-1}^{\omega_{T-1}}\right)\right)
$$

where

$$
\left(\sigma_{T-1}^{\omega_{T-1}}\right)^{2}:=\operatorname{var}\left(\bar{z}_{T-1}^{\omega_{T-1}}\right)=\sum_{l_{T-1}=1}^{L_{T-1}}\left(\rho_{T-1}^{\omega_{T-1}, l_{T-1}}\right)^{2}\left(\sigma_{T-1}^{l_{T-1}}\right)^{2}
$$

The distribution of the stage $T-1$, scenario $\omega_{T-1}$ expected costs, $\tilde{z}_{T-1}^{\omega_{T-1}}$, is induced by the estimation errors in the cuts which have been obtained so far in stage $T-1$. The distribution is different for different scenarios $\omega_{T-1}$ due to the different dual variables $\rho_{T-1}^{\omega_{T-1}, l_{T-1}}$.

Next, we discuss the general case of the relation between stage $t-1$ and stage $t$, $t=2, \ldots, T-1$. In this case, the subproblems in stage $t$ contain cuts which have been computed by Monte Carlo importance sampling and therefore represent an estimate of the outer linearization of the future stage $t+1$ expected costs. 


\section{The Stage $t$ Subproblem:}

$$
\begin{aligned}
& \tilde{z}_{t}^{\omega_{t}}=\min c_{t} x_{t}^{\omega_{t}}+\theta_{t}^{\omega_{t}} \\
& s / t \\
& \pi_{t}^{\omega_{t}}: \quad A_{t} x_{t}^{\omega_{t}} \quad=b_{t}^{\omega_{t}}+B_{t-1}^{\omega_{t}} \hat{x}_{t-1} \\
& \rho_{t}^{1, \omega_{t}}: \quad-\bar{G}_{t}^{1} x_{t}^{\omega_{t}}+\theta_{t}^{\omega_{t}} \geq \bar{g}_{t}^{1}+\epsilon_{t}^{1} \\
& \rho_{t}^{L_{t}, \omega_{t}}: \quad-\bar{G}_{t}^{L_{t}} x_{t}^{\omega_{t}}+\theta_{t}^{\omega_{t}} \geq \bar{g}_{t}^{L_{t}}+\epsilon_{t}^{L_{t}} \\
& x_{t}^{\omega_{t}} \quad \geq 0 \text {. }
\end{aligned}
$$

In the analysis of the stage $T-1$ master problem we concluded that the objective value in stage $T-1$, scenario $\omega_{T-1}, \tilde{z}_{T-1}^{\omega_{T-1}}$, is normally distributed with mean $\bar{z}_{T-1}^{\omega_{T-1}}$ and variance $\left(\sigma_{T-1}^{\omega_{T-1}}\right)^{2}=\operatorname{var}\left(\bar{z}_{T-1}^{\omega_{T-1}}\right)$. In the following we show that for any stage $t$, $t=2, \ldots, T-2$, the expected costs $\tilde{z}_{t}^{\omega_{t}}$ are normally distributed with mean $\bar{z}_{t}^{\omega_{t}}$ and variance $\operatorname{var}\left(\bar{z}_{t}^{\omega_{t}}\right)$, which we will denote by $\left(\sigma_{t}^{\omega_{t}}\right)^{2}$ :

$$
\tilde{z}_{t}^{\omega_{t}}=N\left(\bar{z}_{t}^{\omega_{t}},\left(\sigma_{t}^{\omega_{t}}\right)^{2}\right)
$$

where

$$
\left(\sigma_{t}^{\omega_{t}}\right)^{2}:=\operatorname{var}\left(\bar{z}_{t}^{\omega_{t}}\right)=\sum_{l_{t}=1}^{L_{t}}\left(\rho_{t}^{\omega_{t}, l_{t}}\right)^{2}\left(\sigma_{t}^{l_{t}}\right)^{2}
$$

and

$$
\left(\sigma_{t}^{l_{t}}\right)^{2}:=\operatorname{var}\left(\bar{z}_{t+1}\left(\hat{x}_{t}^{l_{t}}\right)\right)
$$

In order to do so, we discuss the relation between stage $t-1$ and stage $t$, where the stage $t, \omega_{t}$, problems are subproblems of the stage $t-1, \omega_{t-1}$ master problem. Given a solution $\hat{x}_{t-1}$ passed from the current stage $t-1, \omega_{t-1}$ master problem, the stage $t$ costs, $\tilde{\tilde{z}}_{t}$, are distributed both with respect to $\omega_{t}$ and with respect to the error distributions $\epsilon_{t}$ of the right-hand sides of the cuts.

$$
\tilde{\tilde{z}}_{t}=\tilde{\tilde{z}}_{t}\left(\omega_{t}, \epsilon_{t}^{1}, \ldots, \epsilon_{t}^{L_{t}}\right) .
$$

The expected value of the stage $t$ costs with respect to the $\omega$ distribution, $\tilde{z}_{t}$, can be computed as

$$
\tilde{z}_{t}=E_{\omega_{t}}\left(\tilde{\tilde{z}}_{t} \mid \omega_{t}\right)=E_{\omega_{t}}\left(\tilde{z}_{t}^{\omega_{t}}\right)
$$

using Monte Carlo importance sampling. Remember that $\tilde{z}_{t}^{\omega_{t}}=N\left(\bar{z}_{t}^{\omega_{t}}, \operatorname{var}\left(\bar{z}_{t}^{\omega_{t}}\right)\right)$. We compute the mean value $\bar{z}_{t}^{\omega_{t}}$ by substituting the mean value of 0 of the error distributions $\epsilon_{t}^{l_{t}}$. That means, the current estimate of the mean is the current objective value.

We compute the variance $\operatorname{var}\left(\bar{z}_{t}\right)$ based on conditional expectations:

$$
\begin{aligned}
\operatorname{var}\left(\tilde{\tilde{z}}_{t}\right) & =E_{\omega_{t}}\left(\operatorname{var}\left(\tilde{\tilde{z}}_{t}\right) \mid \omega_{t}\right)+\operatorname{var}_{\omega_{t}}\left(E_{\epsilon_{t}} \tilde{\tilde{z}}_{t} \mid \omega_{t}\right) \\
& =E_{\omega_{t}} \operatorname{var}\left(\tilde{z}_{t}^{\omega_{t}}\right)+\operatorname{var}_{\omega_{t}}\left(\bar{z}_{t}^{\omega_{t}}\right)
\end{aligned}
$$




$$
\begin{aligned}
& \operatorname{var}\left(\bar{z}_{t}\right)=E_{\omega_{t}} \operatorname{var}\left(\bar{z}_{t}^{\omega_{t}}\right)+\frac{1}{n} \operatorname{var}_{\omega_{t}} \bar{z}_{t}^{\omega_{t}} \\
& \left(\sigma_{t, C U T}\right)^{2} \quad\left(\sigma_{t, S A M P L}\right)^{2} \\
& \text { future current. }
\end{aligned}
$$

The variance of the estimated mean of the stage $t$ costs can be seen as being composed of a sum of two terms. The first term concerns the influence of the error of the estimated cuts. It is an aggregate of the estimation error of all future periods, $t+2, \ldots, T$. We refer to it as the future term of the estimation error. Clearly, $\operatorname{var}\left(\bar{z}_{t}^{\omega_{t}}\right)=\sum_{l_{t}=1}^{L_{t}}\left(\rho_{t}^{\omega_{t}, l_{t}}\right)^{2}\left(\sigma_{t}^{l_{t}}\right)^{2}$. The second term concerns the current estimation error due to using sampling in stage $t$. In the case of two-stage problems, only the second term appears because the subproblems do not contain cuts estimated by Monte Carlo importance sampling. Given $\hat{x}_{t-1}$ passed from the stage $t-1$ master problem, we can define the variance of the estimate $\operatorname{var}\left(\bar{z}_{t}\right)$ to be

$$
\left(\sigma_{t-1}^{l_{t-1}}\right)^{2}:=\operatorname{var}\left(\bar{z}_{t}\left(\hat{x}_{t-1}^{l_{t-1}}\right)\right)
$$

Thus, the expected value of the stage $t$ costs is normally distributed with mean $\bar{z}_{t}\left(\hat{x}_{t-1}^{l_{t-1}}\right)$ and variance $\left(\sigma_{t-1}^{l_{t-1}}\right)^{2}$

$$
\tilde{z}_{t}\left(\hat{x}_{t-1}^{l_{t-1}}\right):=N\left(\bar{z}_{t}\left(\hat{x}_{t-1}^{l_{t-1}}\right),\left(\sigma_{t-1}^{l_{t-1}}\right)^{2}\right) \text {. }
$$

The error correction terms of the stage $t-1$ cuts are represented as

$$
\epsilon_{t-1}^{l_{t-1}}=N\left(0,\left(\sigma_{t-1}^{l_{t-1}}\right)^{2}\right)
$$

where we assume that the error distribution is approximately constant with respect to $x_{t-1}$. The minimum costs in stage $t-1$, scenario $\omega_{t-1}, z_{t-1}^{\omega_{t-1}}$ are normally distributed with mean $\bar{z}_{t-1}^{\omega_{t-1}}$ and variance $\left(\sigma_{t-1}^{\omega_{t-1}}\right)^{2}$ :

$$
\tilde{z}_{t-1}^{\omega_{t-1}}=N\left(\bar{z}_{t-1}^{\omega_{t-1}},\left(\sigma_{t-1}^{\omega_{t-1}}\right)^{2}\right)
$$

Knowing the distribution of the error terms of the cuts in each stage $t, t=$ $2, \ldots, T-1$, we can compute an estimate of the mean of the stage $t$ costs and the corresponding estimated variance of the mean value of the costs.

At stage 1 we obtain an estimate $\bar{z}_{1}$ with corresponding variance $\operatorname{var}\left(\bar{z}_{1}\right)$, where we define

$$
\left(\sigma_{1}\right)^{2}:=\operatorname{var}\left(\bar{z}_{1}\right)
$$

and

$$
\left(\sigma_{1}\right)^{2}=\sum_{l_{1}=1}^{L_{1}}\left(\rho_{1}^{l_{1}}\right)^{2}\left(\sigma_{1}^{l_{1}}\right)^{2} .
$$

The distribution of the optimal first stage costs,

$$
\tilde{z}_{1}=N\left(\bar{z}_{1},\left(\sigma_{1}\right)^{2}\right),
$$

represents a lower bound estimate for the expected costs of the multi-stage stochastic linear program. 


\subsubsection{The Upper Bound Estimate}

To obtain an upper bound for the total expected costs of the multi-stage problem, we evaluate the expected costs of the current first-stage trial solution $\hat{x}_{1}$. This can be accomplished by sampling paths from stages $2, \ldots, T$. For a reference, see Pereira and Pinto (1989) [103]. To efficiently sample a small number of paths so as to obtain an accurate estimate of the expected costs associated with $\hat{x}_{1}$, we also use importance sampling. We define a path $\hat{s}^{\omega}=\left(\hat{x}_{1}, \hat{x}_{2}, \ldots, \hat{x}_{T}\right)^{\omega}, \omega \in \Omega$, where $\Omega=\left\{\Omega_{12} \times \Omega_{3} \times\right.$ $\left.\ldots \times \Omega_{T}\right\}$, as a sequence of optimal solutions $\hat{x}_{t}^{\omega_{t}}$ of stage $t$, scenario $\omega_{t}$ problems, $t=2, \ldots, T$, with $\hat{x}_{1}$ being the first- stage trial solution. A path is computed by following the "wait-and-see" paradigm. We pass $\hat{x}_{1}$ to the second stage and solve the second-stage problem for scenario $\omega_{2}$ to obtain the optimal solution $\hat{x}_{2}^{\omega_{2}}$. Next we pass the obtained second- stage solution $\hat{x}_{2}^{\omega_{2}}$ to the third-stage and solve the thirdstage problem for scenario $\omega_{3}$ to obtain $\hat{x}_{3}^{\omega_{3}}$. We continue in this way until we obtain $\hat{x}_{T}^{\omega_{T}}$ in stage $T$. Note that when solving the stage $t$ problem, no future outcomes $\omega_{t+1}, \ldots, \omega_{T}$ are used. All future information at each stage is solely represented by means of the cuts added in stage $t$ so far. The costs of a path $\hat{s}^{\omega}, C\left(\hat{s}^{\omega}\right)$, is given by $C\left(\hat{s}^{\omega}\right)=\sum_{t=1}^{T} c_{t} \hat{x}_{t}^{\omega_{t}}$. The expected value of the costs of all paths $\hat{s}^{\omega}, E \hat{s}^{\omega}$ gives an upper bound for the costs of a trial solution $\hat{x}_{1}$. Figure 4.2 represents schematically five paths sampled through the four-stage problem of Figure 4.1.

We sample paths by applying the importance sampling scheme to the space of dimension $\sum_{t=2}^{T} h_{t}$ of all random parameters $V_{t, i_{t}}, i_{t}=1, \ldots, h_{t}, t=2, \ldots, T$. For sampling paths the importance density $q(V)$ is computed based on the additive marginal approximation function analogous to the way it was defined in Section 2.3.3 in Chapter "Benders Decomposition and Importance Sampling for Stochastic Linear Programs":

$$
\Gamma(V)=C(\tau)+\sum_{t=1}^{T} \sum_{i_{t}=1}^{h_{t}} C\left(\tau_{1,1}, \ldots, \tau_{t, i_{t}-1}, V_{t, i_{t}}, \tau_{t, i_{t}+1}, \ldots, \tau_{T, h_{T}}\right)-C(\tau),
$$

where $V=\left(V_{1}^{1}, \ldots, V_{h_{1}}^{1}, V_{1}^{2}, \ldots, V_{h_{\mathrm{t}}}^{T}\right)$ and $\tau=\left(\tau_{1}^{1}, \ldots, \tau_{h_{1}}^{1}, \tau_{1}^{2}, \ldots, \tau_{h_{\mathrm{t}}}^{T}\right)$. Using importance sampling for the upper bound estimate, we hope to obtain accurate estimates with a small sample size. Note that the advantage of sampling paths lies in the fact that we only linearly increase the number of sample points with the number of stages, whereas the decision tree grows exponentially with the number of stages.

\subsection{THE ALGORITHM}

By solving a sample of subproblems $\omega_{t+1}$ according to the importance sampling scheme we compute estimates of the expected future costs $z_{t+1}^{\omega_{t}}$ and of the gradients $G_{t}^{l_{t}}$ and right-hand sides $g_{t}^{h_{t}}$ of the cuts in each stage $t$ and scenario $\omega_{t}$. The optimal objective function value for each stage $t$, scenario $\omega_{t}$ subproblem gives an estimated lower-bound for the expected costs $z_{t}^{\omega_{t}}=c_{t} \hat{x}_{t}^{\omega_{t}}+\hat{\theta}_{t}^{\omega_{t}}$, subject to scenario $\omega_{t}$ and subject to $\hat{x}_{t-1}$, the (optimal) solution passed forward from the previous stage. The obtained lowerbound estimate is the tightest lower bound that can be generated, if in stage $t+1$ 


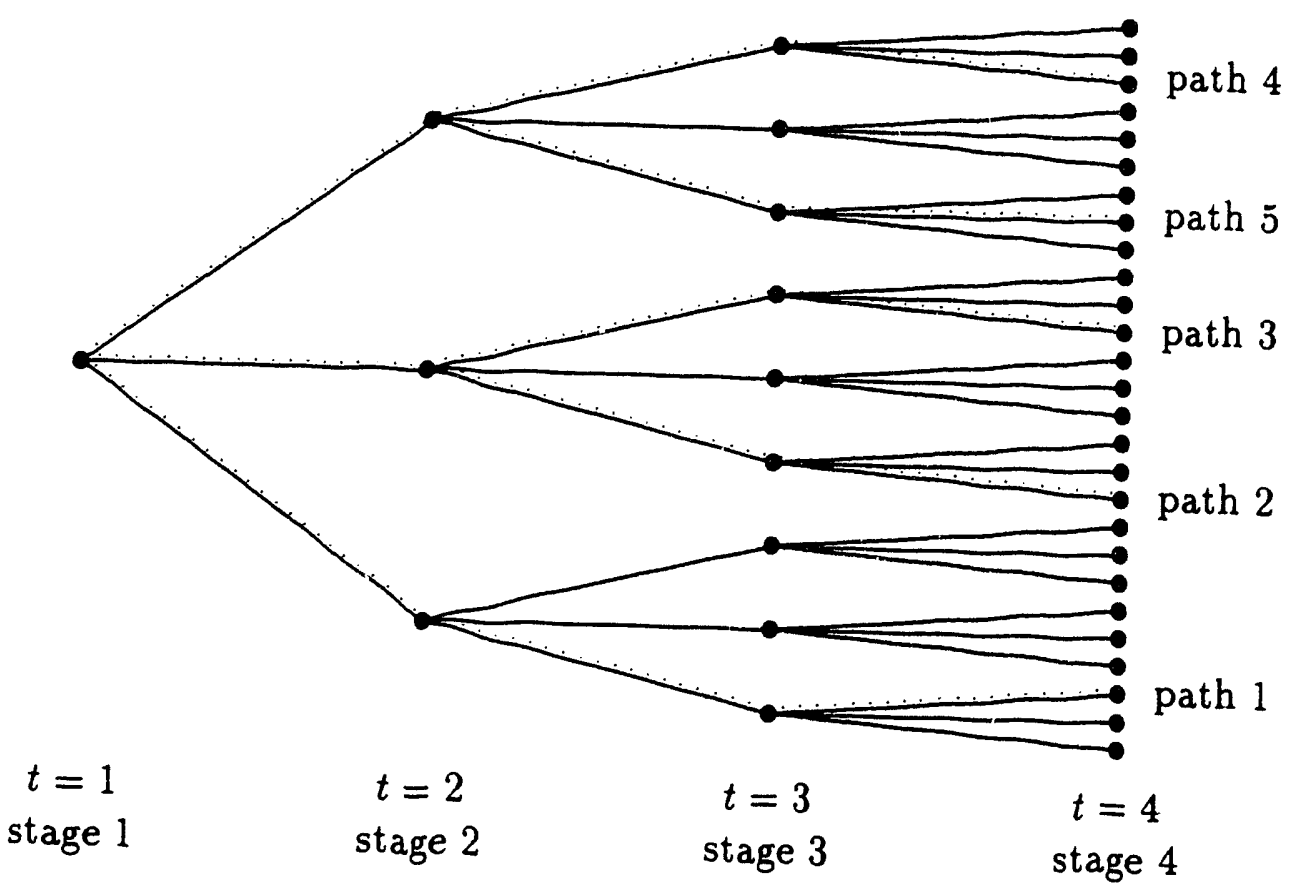

Figure 4.2: Path sampling for upper bounds

a sufficient number of cuts have been added to represent the expected future costs with respect to stage $t+2$ for all scenarios $\omega_{t+1} \in \Omega_{t+1}$ and is a weaker lower-bound estimate if there is not a sufficient number of cuts.

We are especially interested in the lower-bound estimate of the first-stage costs, which we obtain by solving the first-stage problem. If the first-stage problem is balanced with the second stage (that is, if the cuts added so far to the first stage prublem fully represent the expected second-stage costs), and if the second-stage is balanced with the third stage for all scenarios $\omega_{2} \in \Omega_{2}$ and all values of $\hat{x}_{1}$, and so forth until stage $T-1$, then the solution of the first-stage problem is the optimum solution of the multi-stage stochastic linear program. In this case, the lower bound estimate of $z_{1}$ takes on the value of the total expected costs of the multi-stage problem.

To estimate an upper bound for the total expected costs of the multi-stage problem, we use the path-sampling scheme with importance sampling to evaluate the expected costs of the current first-stage trial decision $\hat{x}_{1}$. Sampling paths $\omega \in \Omega$ according to this importance sampling scheme, we obtain an equal number of sample points $\omega_{t} \in \Omega_{t}$ in stages $t=2, \ldots, T$. Figure 4.3 represents schematically these sample points for the example of the five paths of Figure 4.2. At these sample points 


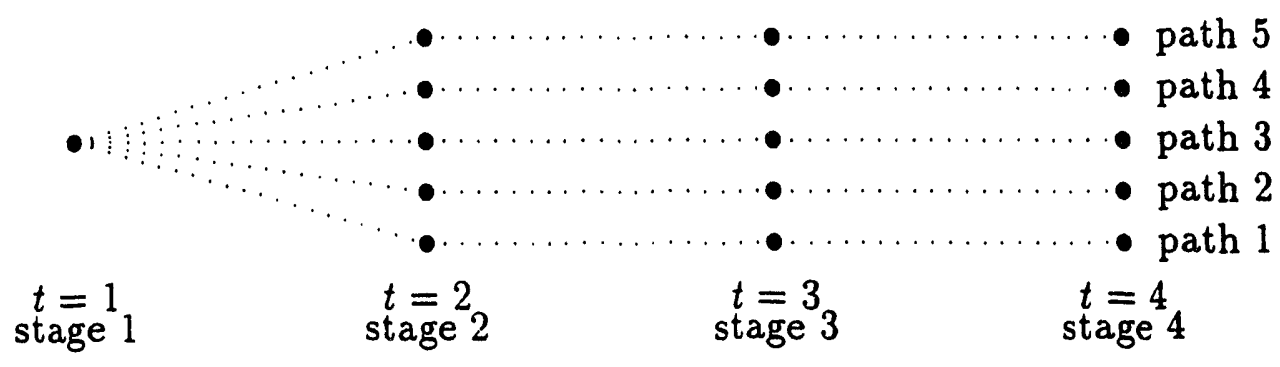

Figure 4.3: Sample points obtained from path sampling

we define the current stage $t$ scenario $\omega_{t}$ subproblems and generate cuts to be added at stages $t=1, \ldots, T-1$ by employing importance sampling as described above for cuts.

The overall procedure works as follows. Solving the stage 1 problem at iteration 1 , we obtain a trial solution $\hat{x}_{1}$ and a lower bound estimate for the expected costs $z_{1}$. Now we employ the path sampling procedure to obtain an upper bound estimate for the expected costs $z_{1}$. If the upper bound estimate and the lower bound estimate are within a given optimality tolerance, we call the first-stage solution the optimal solution of the multi-stage problem and quit. Otherwise, we generate cuts in stages $1, \ldots, T-1$. The path-sampling procedure used for the upper bound estimate has produced sample points $\omega_{t} \in \Omega_{t}$ in stages $t=2, \ldots, T$ with corresponding ancestor solutions $\hat{x}_{1}$ and $\hat{x}_{t}^{\omega_{t}}$ in stages $t=2, \ldots, T-1$ to be passed to the current stage $t$ scenario $\omega_{t}$ problem. Starting at stage $T-1$ and moving backwards to stage 1 , we take each sample problem $\omega_{t}$ in stage $t$ and finally the stage 1 problem as the current master problem and compute cuts by again sampling $\omega_{t+1} \in \Omega_{t+1}$ descendant subproblems until each scenario problem $\omega_{t}$ in stage $t$ is balanced with stage $t+1$ with respect to ancestor solutions $\hat{x}_{t-1}$ which have been passed from stage $t-1$. Arriving at stage 1 , we obtain a new solution $\hat{x}_{1}$ and a new lower bound estimate. We continue as defined above by sampling new paths for the upper bound estimate. Finally, after a finite number of iterations, upper and lower bound estimates will be sufficiently close. Upper and lower bound estimates can be seen as the sum of i.i.d. random terms which for sample sizes of 30 or more can be assumed normally distributed with known variances (derived from the sampling process). A $95 \%$ confidence interval for the obtained solution is computed. 


\subsection{COMPUTATIONAL EXPERIENCE}

Computational results from using Benders decomposition and importance sampling for two-stage problems and a special class of multi-stage problems are discussed in Section 2.7 in Chapter "Benders Decomposition and Importance Sampling for Stochastic Linear Programs" where we report on the solution of test problems with up to 52 stochastic parameters and a number of universe scenarios exceeding $10^{24}$. Using importance sampling and small sample sizes (between 200 and 600) very accurate results were obtained. Additional tests on these examples showed that the variance reduction factor obtained by using importance sampling versus crude (naive) Monte Carlo sampling was up to about $10^{-6}$.

Inspired by these results, we implemented an earlier version of the methodology described above for the multi-stage case which did not consider dependency between stages. Instead of the path-sampling procedure for obtaining upper bound estimates, we implemented a procedure of sampling points which required handling of exponentially growing decision trees. Therefore, even when we used very small sample sizes, the number of stages that was practical to solve was limited.

We did test up to 3-stage problems. FI3 is a 3-stage test problem derived from a 2-stage financial portfolio problem found in Mulvey and Vladimirou (1989) [96]. The problem is to select a portfolio which maximizes expected returns in future periods, taking into account the possibility of revising the portfolio in each period. There are transaction costs and bounds on the holdings and turnovers. Our test problem covers a planning horizon of 3 periods whereas the original Mulvey-Vladimirou test problem was a 2-stage problem that compressed all future periods into a single second stage. They solved the stochastic problem by restricting the number of scenarios.

We assumed the returns of the stocks in the future periods to be independent stochastic parameters with 3 outcomes each. With 13 assets with uncertain returns, the problem had 26 stochastic parameters instead of 39 , because after the last- stage decision is made, the expected money-value of the portfolio can be evaluated. The number of universe scenarios was $2.5 \cdot 10^{12}$. (The deterministic equivalent formulation of the problem would have more than $10^{\mathbf{1 4}}$ rows and a similar number of columns.) We obtained an estimated optimal solution of the 3-stage stochastic problem using a sample size of only 50 per stage. The optimal objective function value was estimated to be 1.10895 with an estimated $95 \%$ confidence in .rval of $0.004 \%$ on the leit side and $0.001 \%$ on the right side of the obtained objective function value. Thus the optimal objective value lies within $1.10881 \leq z^{*} \leq 1.10895$ with $95 \%$ probability. Note how small the confidence interval is. 


\subsection{APPLICATIONS OF MULTI-STAGE STOCHASTIC LINEAR PROGRAMS}

In the following we discuss two potential applications of multi-stage stochastic linear programs, one in the area of operations planning of power systems and the other in the area of portfolio optimiration.

\subsubsection{The Control of Hydro Power Systems}

An important problem is the control of the short-term operation of hydro power systems. A river system can be described as a network of river basins, where outflows from one basin are inflows to another basin down the river. Powerhouses with different numbers of turbine-generator blocks operating in parallel for producing electrical energy are associated with each of the basins. There are two different types of outflows from basins, poweihouse (or turbine) flows and spill flows. The former go through the turbines and are used for energy generation; the latter bypass the turbines and cannot be used for energy generation. Spill is not desired in regular operation and is only used in situations when basin capacities and powerhouse-flow capacities are reached. Inflows into basins either result from outflows from basins up the river or are additional exogenous inflows. The exogenous inflows include all natural inflows into the river system (for example, rivers not used for power generation) and inflows resulting from reservoirs operated independently of the system under consideration.

The operating environment can be stated as follows. The river system is operated by a local load dispatcher as an independent economic entity. Energy produced by the river system is transmitted into the high-voltage system of a higher level power company (producer, transmitter and distributor) operated by the principal load dispatcher. The local load dispatcher receives requests for energy production from the principal load dispatcher. Prices paid depend on marginal cost calculations and vary with season and time of day. The local load.dispatcher has to determine a schedule of operation for the river system which fulfills the requests of the principal load dispatcher, complies with various restrictions on the operation, and is based on projections of exogenous inflows and planned operation of independently operated reservoirs whose outflows are inflows to the river system.

Longer-term operations planning is conducted based on optimizations of planning horizons of a week, a day or a weekend. Results from these longer-term optimizations influence short-term control by defining start and end values for reservoir levels. In fact the local load dispatcher determines a rough schedule of the operation of the system for the next day based on planned energy requests and projections of inflows. Based on this rough daily schedule, on actual changes of the planned requests, and on short-term hydrological projections of inflows, the operation of the system has to be controlled for the immediate future. This means controlling actual operation of the system for the next four hours.

Predictions of natural inflows are usually based on the mean value of inflows of the 
previous day, the actually measured current value of inflow, and the hydrological forecast. While hydrological forecasts are very inaccurate when extended to several days ahead, they can be reasonably good for shorter horizons, for example the next day. However, there is still important uncertainty involved as the hydrological forecasts depend heavily upon local weather conditions, which usually cannot be predicted sufficiently accurately. Inflows resulting from the operation of independently operated reservoirs are also subject to uncertainty because changes in electricity demand may result in a different schedule than the one predicted beforehand.

The control of the system has to have the ability to hedge against the stochastic nature of water availability. It has to protect against situations of shortage so that a sudden lack of inflow does not result in being unable to neet energy demand requests. It also has to be able to take advantage of situations of surplus inflow, so that a sudden, unforeseen increase in inflow does not result in having to spill water without being able to use it to produce electricity. It is clear that the system has to be able to adapt. That means it has to be able to observe when inflows differ from those projected and be able to take action accordingly.

The short-term control of the operation of the system continuously determines the levels of all turbine flows and spill flows. Given turbine and spill flows and given actual observations of all exogenous flows, all other variables of the hydraulic system are determined, for example, the volumes and levels of water in the reservoirs. Also, given turbine and spill flows and given the characteristics of the turbines and generators, the electric power produced by each generator is determined as well as the total electric power produced by the river system as a whole.

The relations between the different variables are not necessarily linear. The volume versus level characteristic of a reservoir is non-linear and depends on the shape of the reservoir. The power of the turbine depends on the turbine flow and the head, i.e., the height difference between the levels of the upper and lower reservoir. Even for constant head, the relation between turbine flow and power produced is non-linear. The efficiency of a generator depends non-linearly on the power produced by the turbine. The flow into and out of a reservoir involves certain delays (the time that water entering the reservoir takes until it is available at the turbines), which in turn depend on the water level in the reservoir. In river segments between reservoirs, the velocity of the water flow depends on its volume. Each machine requires a certain minimum power for operation, for example a machine can either be operated with at least a certain level of power or has to be shut down and not operated at all. Also the velocity of load changes in turbines may have to be restricted. The latter operational constraints are non-convex.

The non-linear and non-convex relations of practical problems can be tackled by using mixed-integer formulations which represent non-linearities by piecewise linear functions with a sufficient number of linear segments.

Let $x_{i}(t), i=1, \ldots, k$, be the reservoir volumes of the $k$ reservoirs of the hydrJ system, $\bar{u}_{i}(t), i=1, \ldots, k$, the spill flows from the reservoirs $i, u_{i}(t), i=1, \ldots, k$, 
be the turbine flows through the turbines of reservoir $i$, and $\tilde{q}_{i}(t)$ be the exogenous inflows into each reservoir of the hydro system. Let $y_{i}(t), i=1, \ldots, k$, be the electric power produced at each reservoir and $y(t)$ be the total electric power produced by the system at time $t$. The optimal control problem of the hydro power system will be formulated in terms of these variables.

In order to not to blur the insight into the problem by too many details, we make a few simplifications in the formulation presented below. As a river system, we consider a series of reservoirs. We consider constant flow times $\tau_{i}$, we do not take into account minimum load requirements for the operation of generator-turbine units, and we formulate the functions $f_{i}$, which relate the electricity generated to the turbine flows and the head of the reservoir, by a piecewise linear representation. Under these simplifications, the hydro system can be represented by a system of linear differential equations, and the problem appears as linear optimal control problem with a fixed end-point.

$$
\max \int_{0}^{T} c(t) y(t) d t
$$

subject to

$$
\begin{aligned}
& i=1, \ldots, k: \\
& \dot{x}_{i}(t)=\tilde{q}_{i}(t)-\bar{u}_{i}(t)-u_{i}(t)+\bar{u}_{i-1}\left(t-\tau_{i}\right)+u_{i-1}\left(t-\tau_{i}\right), u_{0}(t)=\bar{u}_{0}(t)=0, \\
& x_{i}(0)=x_{i}^{0}, x_{i}(T)=x_{i}^{T}, \text { given, } \\
& u_{i}^{\min } \leq u_{i}(t) \leq u_{i}^{\max }, \bar{u}_{i}^{\min } \leq \bar{u}_{i}(t), \\
& y_{i}(t)=f_{i}\left(x_{i}, x_{i+1}, u_{i}\right), \\
& y(t)=\sum_{i=1}^{k} y_{i}(t), \\
& y^{\min }(t) \leq y(t) \leq y^{\max }(t) .
\end{aligned}
$$

Note that the inflows $\tilde{q}_{i}(t)$ are uncertain parameters. Initially only a forecast of the inflows is available. Later, after some time, observations of the random parameters are made. As the control system is supposed to be able to learn, it has to be able to recognize differences between the expected values and later observed outcomes of inflows and adjust the control decisions, $u_{i}(t)$ and $\bar{u}_{i}(t)$.

Instead of solving the problem directly as a continuous time problem, we discretize the planning horizon into $T$ discrete time steps. The choice of time-step width depends on the particular model to be solved and its parameters. For example, in our application to hydro power systems control, we will probably choose a time-step width of about 10 minutes. The planning horizon of 3 hours then breaks down into 24 time steps.

By discretizing, the system of linear differential equations can be transformed into a system of linear equations, and the optimal control problem can be stated as 
a multi-stage dynamic linear program. The random right hand sides correspond to uncertain exogenous inflows. When the control problem is stated and solved only the predicted values of inflows are known. Due to prediction error the forecasted values of inflows are random parameters. We can assume that their distribution is known.

In general, we expect dependency between the stochastic parameters both within a certain stage, and between stages. We describe correlation of random parameters within a stage by a linear relation, e.g. outcomes of the $k$ uncertain inflows $\tilde{q}_{t}$ can be obtained by multiplying outcomes of a vector of independent random parameters $V=\left(V_{1}, \ldots, V_{h}\right)$ by a matrix $F(k \times h)$, thus

$$
\tilde{q}^{t}=F V^{t}
$$

We consider inter-period dependency as a Markovian process (additive dependency):

$$
\tilde{q}^{t}=F V^{t}+H \tilde{q}^{t-1}
$$

where $H(k \times k)$. The value of random parameter $i$ in period $t$ is a weighted sum of the values of the random parameters in the previous period $t-1, \tilde{q}^{t-1}$, plus a weighted sum of some independent random variation in the current period $t, V^{t}$. The parameters of this linear additive correlation model can be estimated based on historical observations.

\subsubsection{The Multi-Period Asset Allocation Problem}

In Dantzig and Infanger (1991) [28] we formulated a class of multi-period financial asset allocation problems related to Mulvey and Vladimirou (1989) [96] and showed how they can be solved by adaptations of multi-stage stochastic linear programing methodology. We now outline this application.

At time period 1 a certain amount of wealth is available to a decision maker invested in assets $i=1, \ldots, n$, and in cash which we index as asset $n+1$. We denote $\bar{x}_{i}, i=1, \ldots, n+1$, to be the dollar value of the initially available assets. The decision maker has to decide each period how to rearrange his portfolio so to achieve the best return on his initial investment over time. We consider the problem in discrete time and define time steps $t=1, \ldots, T$, with $T$ being the end of the planning horizon.

At each time period $t$, the investor can either maintain the level of asset $i$, buy more, or sell off part (or all) of it. We denote by $y_{i}^{t}$ the amount sold of asset $i$ in period $t$ and by $x_{i}^{t}$ the amount of asset $i$ retained in period $t$. Selling asset $i$ means decreasing the value of $x_{i}^{t}$ and increasing the value of cash, $x_{n+1}^{t}$. Also, the investor has the choice of using available cash to buy certain amounts of assets $i$. The amount bought in period $t$ is denoted by $z_{i}^{t}$.

Buying and selling entails transaction costs which we assume to be proportional to the dollar value of the trade. We denote by $100 \nu_{i}$ the transaction costs (expressed as a percentage) associated with buying one unit of asset $i$ and by $100 \mu_{i}$ the transaction costs (expressed as a percentage) associated with selling 1 unit of asset $i$. Buying 1 
unit of asset $i$ requires $1+\nu_{i}$ units of cash, and selling 1 unit of asset $i$ returns $1-\mu_{i}$ units of cash.

Through buying and selling the investor can restructure his portfolio in each time period $t$. Once this stage $t$ decision is made, the post-trade holdings $x_{i}^{t}, i=1, \ldots, n+$ 1 , can be calculated. The shares in the portfolio are then kept constant until the next time period. The value of $x_{i}^{t}$ is affected by the returns on the market. For example, a holding of $x_{i}^{t}$ at time $t$ changes in value to $R_{i}^{t} x_{i}^{t}$, where $R_{i}^{t}$ denotes the return factor from period $t$ to period $t+1$.

At time $t$, when the decision on rearranging the portfolio has to be made, returns $R_{i}^{t}$, for $i=1, \ldots, n$ are not known to the decision maker with certainty. Only the return on cash, $R_{n+1}^{t}$ is assumed known. However, we assume we know the probability distributions of the $R_{i}^{t}$. The problem is of the "wait-and-see" type. While the decision in period $t$ has to be made on the basis of distributions of future returns $R_{i}^{s}$, for $i=1, \ldots, n, s=1, \ldots, T$, the values of prior returns $R_{i}^{s}, i=1, \ldots, n, s=1, \ldots, t-1$, have already been observed. We denote by $R^{t}=R_{i}^{t}$, for $i=1, \ldots, n$, the $n$ dimensional random vector of returns, with outcomes $r^{t}\left(\omega_{t}\right), \omega_{t} \in \Omega_{t}$, and corresponding probabilities $p^{\omega_{t}}$. $\Omega_{t}$ is the set of all possible outcomes in $t$. The random returns $R_{i}^{t}$ of period $t$ are mutually dependent and also dependent on the random returns in the previous period.

After the last period $T$, no decision is made. Only the value of the portfolio is determined by adding the values of all assets, including the last-period returns. We call this value $v^{T}$. The goal of the decision maker is to maximize $E u\left(v^{T}\right)$, the expected utility of the value of the portfolio at the end of period $T$. The utility function $u\left(v^{T}\right)$ captures the way the investor views risk. If $u\left(v^{T}\right)$ is linear, it reflects risk neutrality; if $u\left(v^{T}\right)$ is concave, it models risk averseness. Nonlinear utility functions require non linear programming techniques for the solution of the problem. Our methodology is not restricted to linear problems. However, for the sake of ease and computational speed, we approximate the nonlinear function by a piecewise linear function with a sufficiently large number of linear segments.

In the model presented here we do not consider short-selling of assets, although this feature could be incorporated easily. We also do not consider borrowing of cash, which also could be incorporated easily. The holdings of assets, as well as the amounts of assets sold or bought have to be positive. In general there are also lower $(\underline{x})$ and upper $(\bar{x})$ bounds on holdings, as well as on amounts of assets to be sold $(\underline{y}, \bar{y})$ or to be bought $(\underline{z}, \bar{z})$, which are given by the investor and/or by the market. For example, a certain asset may only be available up to a certain amount, or an investor may want to have a certain asset constitute at least a certain amount of dollar value in the portfolio. Therefore in general we formulate $\underline{x}_{i}^{t} \leq x_{i}^{t} \leq \bar{x}_{i}^{t}, \underline{y}_{i}^{t} \leq y_{i}^{t} \leq \bar{y}_{i}^{t}, \underline{z}_{i}^{t} \leq z_{i}^{t} \leq \bar{z}_{i}^{t}$, where $\underline{x}_{i}^{t} \geq 0, \underline{y}_{i}^{t} \geq 0, \underline{z}_{i}^{t} \geq 0, r_{i}^{0} x_{i}^{0}$ given for $i=1, \ldots, n+1, t=1, \ldots, T$.

We can now state the model: 


$$
\begin{aligned}
& t=1, \ldots, T, i=1, \ldots, n+1, r_{i}^{0} x_{i}^{0} \text { given: } \\
& -r_{i}^{t-1} x_{i}^{t-1}+x_{i}^{t}+y_{i}^{t}-\quad z_{i}^{t}=0, i=1, \ldots, n \\
& -r_{n+1}^{t-1} x_{n+1}^{t-1}+x_{n+1}^{t}-\sum_{i=1}^{n}\left(1-\mu_{i}\right) y_{i}^{t}+\sum_{i=1}^{n}\left(1+\nu_{i}\right) z_{i}^{t}=0 \text {, } \\
& -\sum_{i=1}^{n+1} r_{i}^{T} x_{i}^{T}+v^{T}=0, \\
& \max E u\left(v^{T}\right) \\
& \underline{x}_{i}^{t} \leq x_{i}^{t} \leq \bar{x}_{i}^{t}, \quad \underline{y}_{i}^{t} \leq y_{i}^{t} \leq \bar{y}_{i}^{t}, \quad \underline{z}_{i}^{t} \leq z_{i}^{t} \leq \bar{z}_{i}^{t}, \quad i=1, \ldots, n, \quad t=1, \ldots, T .
\end{aligned}
$$

We describe correlation between asset returns using a factor model. Using factors is common in the financial industry (e.g., Perold (1984) [108]); hence, historical data of various factors are commercially available. The idea of the factor model is to relate the vector of asset returns $R^{t}=\left(R_{1}, \ldots, R_{n}\right)^{t}$ to factors $V^{t}=\left(V_{1}, \ldots, V_{h}\right)^{t}$. While the number of assets, $n$, is large (a model should be able to handle about 500 to 3000 assets), the number of factors $h$ is comparatively small. Factor models used in the financial industry typically involve no more than 20 different time series called factors. The $n \times h$ factor matrix $F$ relates $R^{t}$ to $V^{t}$ :

$$
R^{t}=F V^{t}
$$

The coefficients of the factor matrix are estimated using regression analyses on historical data. By linear transformations of historical factors, the transformed factors can always be determined in such a way that the factors $V^{t}$ are orthogonal. These factors can then be interpreted as independent random parameters assumed to be normally distributed or log-normally distributed. Using the factor model, stochastically dependent returns can be generated on the computer by using these stochastically independent factors. We denote an outcome of the random factor $V_{i}^{t}$ by $v_{i}^{t}$, with corresponding probability $p\left(v_{i}^{t}\right):=\operatorname{prob}\left(V_{i}^{t}=v_{i}^{t}\right)$.

We also consider inter-period dependency. For example, we may wish to posit a higher probability of observing a high rate of return in period $t$ if the return was high in period $t-1$ than if it was low in period $t-1$. We can model this inter-period dependency as a Markovian process applied directly on the factors:

$$
v_{i}^{t}=v_{i}^{t-1}+\eta_{i}^{t}, \quad i=1, \ldots, h
$$

The value of factor $i$ in period $t$ is the sum of the value of factor $i$ in period $t-1$ plus some independent random variation of the factor in period $t$, denoted by $\eta_{i}^{t}$. The Markovian model can be estimated based on historical data. Instead of modeling an additive effect as above, we may prefer to model a multiplicative effect by applying the Markovian process directly to the logs of the factors. We have not explored this alternative.

As one can now see easily, the multi-period asset model proposed fits exactly into the framework of a general class of multi-stage stochastic linear programs with 
recourse. The factor model for generating dependent returns and the Markovian process for inter-period dependency define a special class of dependencies between stochastic parameters which we are able to exploit to solve the problem. 


\section{CONCLUSION}

We have discussed and developed a novel approach for solving large-scale stochastic linear programs based on a combination of dual (Benders) decomposition and Monte Carlo importance sampling. Numerical results from large-scale test problems in the areas of facility expansion planning and financial planning demonstrated that very accurate solutions of stochastic linear programs can be obtained with only a small sample size.

The large-scale test problems included various stochastic parameters. For example, the largest problem representing expansion planning for multi-area electric power systems included 39 stochastic parameters. In the deterministic equivalent formulation, if it were possible to state it, the problem would appear as a linear program with about 4.5 billion constraints and variables. The largest portfolio optimization problem included 52 stochastic parameters, which in the deterministic equivalent formulation would appear as a linear program with about $10^{27}$ constraints and a similar number of variables. Problems of these size hitherto seemed to be intractable. Using our method we have been able to solve them on a laptop 80386 computer.

The test results indicate that we have not yet reached the limits of the approach. The sample sizes turned out to be so small that use of parallel processors is not a condition sine qua non for solving even large-scale stochastic linear problems. In order to speed-up the computation time in the case where large sample sizes are required, we have developed a parallel implementation running on a hypercube multi-computer. The numerical results show that speed-ups of about $60 \%$ can be obtained using 64 parallel processors.

Encouraged by the promising numerical results for two-stage and a restricted class of multi-stage problems we have developed the theory for a general class of multi-stage stochastic linear programs. Our approach for solving multi-stage problems, includes special sampling techniques for computing upper bounds and methods of sharing cuts between different sub-problems. It will enable us to efficiently solve large-scale multistage problems with many stages and numerous stochastic parameters in each stage. The implementation is subject to future research. Preliminary numerical results have turned out to be promising.

Further research includes improved decomposition techniques for large-scale problems, e.g., optimized tree traversing strategies and passing information based on nonoptimal subproblems, improvements to the importance sampling approach, e.g., using different types of approximation functions, improved software, e.g., a parallel implementation of the multi-stage algorithm on distributed workstations, and the testing of the methodology on different practical problems in different areas. 


\section{Bibliography}

[1] Abrahamson, P.G. (1983): A Nested Decomposition Approach for Solving Staircase Linear Programs, Technical Report SOL 83-4, Department of Operations Research, Stanford University, Stanford, CA.

[2] Ariyawansa, K.A. and Hudson, D.D. (1990): Performance of a Benchmark Parallel Implementation of the Van Slyke and Wets Algorithm for Two-Stage Stochastic Programs on the Sequent/Balance, Working Paper, Dept. of Pure and Applied Mathematics, University of Washington, Pullman, WA.

[3] Avriel, M., Dantzig, G.B. and Glynn, P.W. (1989): Decomposition and Parallel Processing Techniques for Large-Scale Electric Power System Planning Under Uncertainty, Proceedings of the Workshop on Resource Planning Under Uncertainty, Stanford University, Stanford, CA.

[4] Beale, E.M.L. (1955): On Minimizing a Convex Function Subject to Linear Inequalities, $J$. Roy. Stat. Soc. $17 b, 173-184$.

[5] Beale, E.M.L. Dantzig, G.B. and Watson R.D. (1986): A First-Order Approach to a Class of Multi-Time-Period Stochastic Programming Problems, Mathematical Programming Study 27, $103-117$.

[6] Benders, J.F. (1962): Partitioning Procedures for Solving Mixed-Variable Programming Problems, Numerische Mathematik 4, 238-252.

[7] Ben-Tal, A. and Hochman, E. (1972): More Bounds on the Expectation of a Random Variable, Journal of Applied Probability 9, 809-812.

[8] Birge, J.R. (1984): Aggregation in Stochastic Linear Programming, Mathematical Program$\operatorname{ming} 31,25-41$.

[9] Birge, J.R. (1985): Decomposition and Partitioning Methods for Multi-Stage Stochastic Linear Programming, Operations Research 93, 989-1007.

[10] Birge, J.R. (1980): Solution Methods for Stochastic Dynamic Line ar Programs, Technical Report SOL 80-29, Department of Operations Research, Stanford University, Stanford, CA.

[11] Birge, J.R. and Louveaux F.V. (1985): A Multicut Algorithm for Two-Stage Linear Programs, Technical Report, Department of IOE, University of Michigan, Ann Arbor, MI.

[12] Birge, J.R. and Teboulle, M. (1989): Upper Bounds on the Expected Values of a Convex Function Using Gradient and Conjugate Function Information, Mathematics of Operations Research 14/4, 745-759. 
[13] Birge, J.R. and Wallace, S.W. (1988): A Separable Piecewise Linear Upper Bound for Stochastic Linear Programs, SIAM J. Control and Optimization 26, 3.

[14] Birge, J.R. and Wets, R.J. (1986): Designing Approximation Schemes for Stochastic Optimization Problems, in Particular For Stochastic Programs with Recourse, Math. Progr. Study 27, $54-102$.

[15] Birge, J.R. and Wets, R.J. (1987): Computing Bounds for Stochastic Programming Problems by Means of a Generalized Moment Problem, Mathematics of Operations Research 12, 149162

[16] Birge, J.R. and Wets, R.J. (1989): Sublinear Upper Bounds For Stochastic Programs with Recourse, Mathematical Programming 49, 131-149.

[17] Birge, J.R. and Wets, R.J. (1991) (eds): Stochastic Programming, Proceedings of the 5th International Conference on Stochastic Programming, Annals of Operations Research 30 and 31 .

[18] Charnes, A. and Cooper, A.A. (1959): Chance Constrained Programming, Management Science 6, 73-79.

[19] Cipra, T. (1985): Moment Problem with Given Covariance Structure in Stochastic Programming, Ekonom.-Mat. Obzor 21, 66-77.

[20] Dantzig: G.B. (1948): Programming in a Linear Structure, Comptroller, USAF, Washington, D.C..

[21] Dantzig, G.B. (1955): Linear Programming under Uncertainty, Management Science 1, 197206.

[22] Dantzig, G.B. (1963): Linear Programming and Extensions, Princeton University Press, Princeton, NJ.

[23] Dantzig, G.B. (1988): Planning Under Uncertainty Using Parallel Computing, Annals of Operations Research 14, 1-16.

[24] Dantzig, G.B. and Glynn, P.W. (1990): Parallel Processors for Planning Under Uncertainty, Annals of Operations Research 22, 1-21.

[25] Dantzig, G.B., Glynn, P.W., Avriel, M., Stone, J., Entriken, R. and Nakayama, M. (1989): Decomposition Techniques for Multi-Area Generation and Transmission Planning Under Uncertainty, EPRI Report 2940-1, Electric Power Research Institute, Palo Alto, CA.

[26] Dantzig, G.B., Ho, J.K. and Infanger, G. (1991): Solving Stochastic Linear Programs on a Hypercube Multicomputer, Technical Report SOL 91-10, Department of Operations Research, Stanford University, Stanford, CA.

[27] Dantzig, G.B., and Infanger, G. (1991): Multi-Stage Stochastic Linear Programs for Portfolio Optimization, Technical Report SOL 91-11, Department of Operations Research, Stanford University, Stanford, CA, to appear in Annals of Operations Research.

[28] Dantzig, G.B. and Infanger, G.(1991): Large-Scale Stochastic Linear Programs: Importance Sampling and Benders Decomposition, Technical Report SOL 91-4, Department of Operations Research, Stanford University, Stanford, CA. 
[29] Dantzig, G.B. and Madansky, M. (1961): On the Solution of Two-Staged Linear Programs Under Uncertainty, Proc. 4th Berkeley Symp. on Mathematical Statistics and Probability I, ed. J. Neyman, 165-176.

[30] Dantzig, G.B. and Pereira, M.V.F. (1988): Mathematical Decomposition Techniques for Power System Expansion Planning, EPRI Report EL-5299, Electric Power Research Institute, Palo Alto, CA.

[31] Dantzig, G.B. and Wolfe, P. (1960): The Decomposition Principle for Linear Programs, Operations Research 8, 110-111.

[32] Davis, P.J., and Rabinowitz, P. (1984): Methods of Numerical Integration, Academic Press, London.

[33] Deák, I. (1988): Multidimensional Integration and Stochastic Programming, in Ermoliev, Y. and R.J-B. Wets (eds.): Numerical Techniques for Stochastic Optimization, Springer Verlag, Berlin, Germany, 187-200.

[34] Dempster, M.A.H. (1980): Introduction to Stochastic Programming, in Dempster, M.A.H. (ed.): Stochastic Programming, Academic Press, 3-59.

[35] Dempster, M.A.H. (1986): On Stochastic Programming II: Dynamic Problems Under Risk, Research Report DAL TR 86-5, Dalhousie University, Canada.

[36] Dupačowá, J. (1978): Minimax Approach to Stochastic Linear Programming and the Moment Problem, Zeitschrift für Angewandte Mathematik und Mechanik, 58T, 466-467.

[37] Dupačowá, J. and Wets R.J.-B. (1988): Asymptotic Behavior of Statistical Estimators of Optimal Solutions of Stochastic Optimization Problems, Annals of Mathematical Statistics $16,1517-1549$.

[38] Edmundson, H.P. (1956): Bounds on the Expectation of a Convex Function of a Random Variable, Paper 982, Rand Corporation, Santa Monica, CA.

[39] Entriken, R. (1988): A Parallel Decomposition Algorithm for Staircase Linear Programs, Report ORNL/TM 11011, Oak Ridge National Laboratory, Oak Ridge, TN.

[40] Entriken, R. (1989): The Parallel Decomposition of Linear Programs, Technical Report SOL 89-17, Department of Operations Research, Stanford University, Stanford, CA.

[41] Entriken, R. and Infanger, G. (1990): Decomposition and Importance Sampling for Stochastic Linear Models, Energy, The International Journal, Vol. 15, No 7/8, 645-659.

[42] Ermoliev, Y. (1983): Stochastic Quasi-gradient Methods and Their Applications to Systems Optimization, Stochastics 9, 1-36.

[43] Ermoliev, Y. (1988): Stochastic Quasi-gradient Methods, in Ermoliev, Y. and Wets, R.J-B. (eds.): Numerical Techniques for Stochastic Optimization. Springer Verlag, Berlin, Germany, 141-186.

[44] Ermoliev, Y. (1988): Stochastic Quasigradient Methods and their Implementation, in Ermoliev, Y. and Wets, R.J-B. (eds.): Numerical Techniques for Stochastic Optimization, Springer Verlag, Berlin, Germany, 313-351. 
[45] Ermoliev, Y., Gaivoronski, A. and Nedeva C. (1985): Stochastic Optimization Problems with Partially Known Distribution Functions, SIAM Journal of Control and Optimization 29, 696-716.

[46] Ermoliev, Y. and Wets, R.J-B. (eds.) (1988): Numerical Techniques for Stochastic Optimization, Springer Verlag, Berlin, Germany.

[47] Ferguson, A. and Dantzig, G.B. (1956): The Allocation of Aircraft to Routes: An Example of Linear Programming under Uncertain Demand, Management Science 3, 45-73.

[48] Frauendorfer, K. (1988): Solving SLP Recourse Problems with Arbitrary Multivariate Distributions-The Dependent Case, Mathematics of Operations Research 13, No. 9, 377994.

[49] Frauendorfer, K. (1992): Stochastic Two-Stage Programming, Lecture Notes in Economics and Mathematical Systems 392, Springer Verlag, Berlin, Germany.

[50] Frauendorfer, K. and Kall, P. (1988): Solving SLP Recourse Problems with Arbitrary Multivariate Distributions-The Independent Case, Problems of Control and Information Theory, Vol 17 (4), 177-205.

[51] Gaivoronski, A. (1988): Implementation of Stochastic Quasigradient Methods, in Ermoliev, Y. and Wets, R.J-B. (eds.): Numerical Techniques for Stochastic Optimization, Springer Verlag, Berlin, Germany.

[52] Gaivoronski, A. and Nazereth, L. (1989): Combining Generalized Programming and Sampling Techniques for Stochastic Programs with Recourse, in: Proceedings of the Workshop on Resource Planning Under Uncertainty for Electric Power Systems, Department of Operations Research, Stanford University, Stanford, CA.

[53] Gassmann, H. (1990): MSLiP: A Computer Code for the Multi-Stage Stochastic Linear Programming Problem, Mathematical Programming 47, 407-423.

[54] Gassmann, H. and Ziemba, W.T. (1986): A Tight Upper Bound for the Expectation of a Convex Function of a Multivariate Random Variable, Mathematical Programming Study 27, 39-52.

[55] Gill, P.E., Murray, W. and Wright, M.H. (1981): Practical Optimization, Academic Press, London.

[56] Gill, P.E., Murray, W. and Wright, M.H. (1991): Numerical Linear Algebra and Optimization, Addison-Wesley, Redwood City, CA.

[57] Geoffrion, A.M. (1974): Elements of Large-Scale Mathematical Programming, Management Science 16, No. 11.

[58] Glynn, P.W. and Iglehart, D.L. (1989): Importance Sampling for Stochastics Simulation, Management Science 35, 1967-1992.

[59] Hammersly, J.M. and Handscomb, D.C. (1964): Monte Carlo Methods, Mathuen, London.

[60] Hall, P. (1982): Rates of Convergence in the Central Limit Theorem, Research Notes in Mathematics 62. 
[61] Higle, J.L. and Sen, S. (1989): Stochastic Decomposition: An Algorithm for Two-Stage Linear Programs with Recourse, Mathematics of Operations Research 16/3, 650-669.

[62] Higle, J.L. and Sen, S. (1989): Statistical Verification of Optimality Conditions for Stochastic Programs with Recourse, Annals of Operations Research 30, 215-240.

[63] Higle, J.L., S. Sen, D. Yakowitz (1990): Finite Master Programs in Stochastic Decomposition, Technical Report, Dept. of Systems and Industrial Engineering, The University of Arizona, Tucson, AZ.

[64] Higle, J.L. Wing, L. and Odio, R. (1990): Conditional Stochastic Decomposition: An Algorithmic Interface for Optimization/Simulation. Working Paper 90-009, Dept. of Systems and Industrial Engineering, The University of Arizona, Tucson, AZ.

[65] Glassey, R. (1973): Nested Decomposition and Multi-Stage Linear Programs, Management Science 20, 282-292.

[66] Hiller, R.S. and Eckstein, J. (1990): Stochastic Dedication: Designing Fixed Income Portfolios Using Massively Parallel Benders Decomposition, Working Paper 91-025, Harvard Business School, Cambridge, MA.

[67] Ho, J.K. and Gnanendran, S.K. (1989): Distributed Decomposition of Block-Angular Linear Programs on a Hypercube Computer, College of Business Administration, University of Tennessee, Knoxville, TN.

[68] Ho, J.K., Lee, T.C. and Sundarraj, R.P. (1988): Decomposition of Linear Programs using Parallel Computation, Mathematical Programming 42, 991-405.

[69] Ho, J.K. and Loute, E. (1981): A Set of Staircase Linear Programming Test Problems, Math. Programming 20, 245-250.

[70] Ho, J.K. and Manne, A.S. (1974): Nested Decomposition for Dynamic Models, Math. Programming 6, 121-140.

[71] Huang, C.C., Ziemba, W.T. and Ben-Tal, A. (1977): Bounds on the Expectation of a Convex Function with a Random Variable with Applications to Stochastic Programming, Operations Research 25, 315-325.

[72] Infanger, G. (1990): Monte Carlo (Importance) Sampling within a Benders Decomposition Algorithm for Stochastic Linear Programs, Technical Report SOL 89-13R, Department of Operations Research, Stanford University, Stanford, CA.

[73] Infanger, G. (1991): Monte Carlo (Importance) Sampling within a Benders Decomposition Algorithm for Stochastic Linear Programs, Annals of Operations Research, $\$ 9$.

[74] Intel Corporation (1988): iPSC/2 Fortran Programmer's Reference Manual, Order No. 311019-003.

[75] Intel Corporation, (1898) iPSC/2 Green Hills Fortran Language Reference Manual (Preliminary), Order No. 311020-003.

[76] Jensen, J.L. (1906): Sur les fonctions convexes et les inegalites entres les valeurs moyennes, Acta Mathematica 30, 175-193. 
177] Kall, P. (1974): Approximations to Stochastic Programs with Complete Fixed Recourse, Numer. Math. 22, 393-399.

[78] Kall, P. (1976): Stochastic Linear Programming, Springer Verlag, Berlin, Germany.

[79] Kall, P. (1979): Computational Methods for Two-Stage Stochastic Linear Programming Problems, Angew. Math. Phys. 30, 261-271.

[80] Kall, P., Ruszczynski, A., Frauendorfer, K. (1988): Approximation Techniques in Stochastic Programming, in Ermoliev, Y. and Wets, R.J-B. (Eds.): Numerical Techniques for Stochastic Optimization.

[81] Kall, P. and Stoyan, D. (1982): Solving Stochastic Programming problems with Recourse Including Error Bounds, Mathematische Operationsforschung und Statistik, Series Optimization, 13, 431-447.

[82] King, A. and Wets R.J-B. (1989): EPI-Consistency of Convex Stochastic Programs, submitted to Stochastics.

[83] Krishna, A.S. (1992): Importance Sampling Techniques for Stochastic Linear Programming, Dissertation in progress, Systems Optimization Laboratory, Departm?nt of Operations Research, Stanford University, Stanford, CA.

[84] Kusy, M.I. and Ziemba, W.T. (1986): A Bank Asset and Liability Managoment Model, Operctions Research $34,956-976$.

[85] Lasdon, L. (1970): Optimization Theory for Large Systems, MacMillan, New York, NY.

[86] Lavenberg, S.S. and Welch, P.D. (1981): A Perspective on the Use of Control Variables to Increase the Efficiency of Monte Carlo Simulation, Management Science 27, 322-395.

[87] Louveaux, F.V. (1986): Multi-Stage Stochastic Programs with Block-Seperable Recourse, Mathematical Programming Study 28, 48-62.

[88] Louveaux, F.V. and Smeers, Y. (1988): Optimal Investment for Electricity Generation: A Stochastic Model and a Test Problem, in Ermoliev, Y. and Wets, R.J-B. (Eds.): Numerical Techniques for Stochastic Optimization.

[89] Lustig, I.J, Mulvey, J.M. and Carpenter, T.J. (1991): Formulating Two-Stage Stochastic Programs for Interior Point Methods, Operations Research 39, 757-770.

[90] Madansky, A. (1959): Bounds on the Expectation of a Convex Function a Multivariate Random Variable, Annals of Mathematical Statistics 30, 743-746.

[91] Markowitz, H. (1959): Portfolio Selection: Efficient Diversification of Investments, John Wiley and Sons, New York, NY.

[92] Marti, K. (1980): Solving Stochastic Linear Programs by Semi-Stochastic Approximation Algorithms, in Kall, P. and Prekopa, A. (eds.): Recent Results in Stochastic Programming, Lecture Notes in Economics and Mathematical Systems 179, Springer Verlag, Berlin, Germany, 191-213.

[93] Morton, D.P. (1992): Algorithmic Advances in Multistage Stochastic Programming, Dissertation in progress, Department of Operations Research, Stanford University, Stanford, CA. 
[94] Morton, D.P. and Z. Wang (1992): Benders Decomposition and Tree Traversing Strategies for Solving Multistage Stochastic Linear Programs, Technical Report in Progress, Systems Optimization Laboratory, Department of Operations Research, Stanford University, Stanford, CA.

[95] Mulvey, J.M. (1987): Nonlinear Network Models In Finance, Advances in Mathematica! Programming and Financial Planning, JAI Press, 1, 1987, 253.

[96] Mulvey, J.M. and Vladimirou, H. (1989): Stochastic Network Optimization Models for Investment Planning, Annals of Operations Research 20 (1989) 187-217

[97] Mulvey, J.M. and Vladimirou, H. (1989): Applying the Progressive Hedging Algorithm to Stochastic Generalized Networks, Statistics and Operations Research Series Report, SOR-899, Princeton University, Princeton, New Jersey 08544.

[98] Mulvey, J.M. and Vladimirou, H. (1989): Solving Multi-Stage Stochastic Networks: An A pplication of Scenario Aggregation, Statistics and Operations Research Series Report, SOR-88-1, Department of Civil Engineering and Operations Research, Princeton University, Princeton, NJ.

[99] Murtagh, B.A. and Saunders, M.A. (1983): MINOS 5.0 User's Guide, SOL 83-20, Department of Operations Research, Stanford University, Stanford, CA.

[100] Nazareth, L. and Wets, R.J-B. (1986): Algorithms for Stochastic Programs: The Case of Nonstochastic Tenders, Mathematical Programming Study 28, 48-62.

[101] Nazareth, J.L. and Wets, R.J-B. (1988): Nonlinear Programming Techniques Applied to Stochastic Programs with Recourse, in Ermoliev, Y. and Wets, R.J-B. (eds.): Numerical Techniques for Stochastic Optimization, Springer Verlag, Berlin, Germany, 95-122.

[102] Niederreiter, H. (1986): Multidimensional Numerical Intergration Using Pseudo Random Numbers, Mathematical Programming Study 27, 17-98.

[103] Pereira, M.V. and Pinto, L.M.V.G. (1989): Stochastic Optimization of a Multi-Reservoir Hydro-Electric System-A Decomposition Approach, Water Resources Research 21, 779792.

[104] Pereira, M.V., Pinto, L.M.V.G., Oliveira, G.C. and Cunha, S.H.F. (1989): A Technique for Solving LP Problerns with Stochastic Right-Hand Sides, CEPEL, Centro del Pesquisas de Energia Electria, Rio de Janeiro, Brazil.

[105] Pereira, M.V., Pinto, L.M.V.G., Oliveira, G.C. and Cunha, S.H.F. (1989): A Decomposition Approach to Automated Generation/Transmission Expansion Planning, CEPEL, Centro del Pesquisas de Energia Electria, Rio de Janeiro, Brazil.

[106] Pereira, M.V. and Pinto, L.M.V.G. (1989): Stochastic Dual Dynamic Programming, Technical Note, DEE-PUC/RJ, Catholic University of Rio de Janeiro, Rio de Janeiro, Brazil.

[107] Pereira, M.V.F. and L.M.V.G. Pinto (1991): Multi-stage Stochastic Optimization Applied to Energy Planning, Mathematical Programming 52, 359-375.

[108] Perold, A. (1984): Large-Scale Portfolio Optimization, Management Science 30, 10, 1143. 
[109] Prékopa (1988) Numerical Solution of Probabilistic Constrained Programming Models, in Ermoliev, Y. and Wets, R.J-B. (eds.): Numerical Techniques for Stochastic Optimization, Springer Verlag, Berlin, Germany, 123-139.

[110] Prékopa, A. (1988): Boole-Bonferroni Inequalities and linear Programming, Operations Research $36,145-162$.

[111] Prékopa, A. (1989): Sharp Bounds on Probabilities Using Linear Programming, to appear in Operations Research.

[112] Prékopa, A. (1990): The Discrete Moment Problem and Liriear Programming, Discrete Applied Mathematic 27, 235-254.

[113] Pflug, G.C. (1988): Stepsize Rules, Stopping Times, and their Implementations in Stochastic Quasigradient Algorithms, in Ermoliev, Y. an J Wets, R.J-B. (eds.): Numerical Techniques for Stochastic Optimization, Springer Verlag, Berlin, Germany, 353-372.

[114] Robinson, S.M. and Wets, R.J.-B. (1987): Stability in Two-Stage Stochastic Programming, SIAM J. on Control and Optimization 25, 1409-1416.

[115] Rockafellar, R.T. and Wets, R.J-B. (1989): Scenario and Policy Aggregation in Optimization Under Uncertainty, Mathematics of Operations Research 16, 119-147.

[116] Rubinstein, R.Y. and Marcus, R. (1985): Efficiency of Multivariate Control Variates in Monte Carlo Simulation, Operations Research 39, 661-677.

[117] Ruszczynski, A. (1986): A Regularized Decomposition Method for Minimizing a Sum of Polyhedral Functions, Mathematical Programming 35, 309-933.

[118] Ruszczynski, A. (1987): A Linearization Method for Nonsmooth Stochastic Programming Problems, Mathematics od Operations Research 12, 32-49.

[119] Strazicky, B. (1980): Computational Experience with an Algorithm for Discrete Recourse Problems, in Dempster, M. (ed.): Stochastic Programming, Academic Press, London, 263274.

[120] Tomlin, J. (1973): LPM1 User's Guide, Manuscript, Systems Optimization Laboratory, Stanford University, Stanford, CA.

[121] Van Slyke, R.M. and Wets, R.J-B. (1966): Programming; Under Uncertainty and Stochastic Optimal Control, SIAM J. on Control and Optimization 4, 179-193.

[122] Van Slyke, R.M. and Wets, R.J-B. (1969): L-Shaped Linear Programs with Applications to Optimal Control and Stochastic Programming, SIAM J. of Applied Mathematics 17, 638-663.

[123] Wallace, S.W. (1987): A Piecewise Linear Upper Bound on the Network Recourse Problem, Mathematica: Programming 38, 139-146.

[124] Wets, R.J-B. (1966): Programming under Uncertainty: The Complete Problem, $Z$. Wahrsch. verw. Gebiete 4, 316-339.

[125] Wets, R.J-B. (1974): Stochastic Programs with Fixed Recourse: the Equivalent Deterministic Program, SIAM Review 16, 309-939. 
[126] Wets, R.J-B. (1983): Solving Stochastic Programs with Simple Recourse, Stochastics 10, 219-242.

Uncertainty: The Equivalent Convex Program, SIAM J. on Appl. Math. 14, 89-105.

[127] Wets, R.J-B. (1985): On Parallel Processors Design for Solving Stochastic Programs, Proceedings of the 6th Mathematical Programming Symposium Japanese Mathematical Programming Society, Japan, 13-36.

[128] Wets, R.J-B. (1988): Large-Scale Linear Programming Techniques in Stochastic Programming, in Ermoliev Y. and Wets R.J-B. (eds.): Numerical Techniques for Stochastic Optimization, Springer Verlag, Berlin, Germany.

[129] Wets, R.J-B. (1989): Stochastic Programming, in Nemhauser, G.L., Kan, A.H.G. and Todd, M.J. (eds.): Handbook on Operations Research and Management Science, North-Holland, Amsterdam, The Netherlands, 573-629.

[130] Wittrock, R.J. (1983): Advances in a Nested Decomposition Algorithm for Solving Staircase Linear Programs, Technical Report SOL 83-2, Department of Operations Research, Stanford University, Stanford, CA.

[131] Zenios, S.A. (1990): Massively Parallel Algorithms for Financial Modelling under Uncertainty, Decision Sciences Department, The Wharton School, University of Pennsylvania, Philadelphia, PA.

[132] Zenios, S.A. (1992): A Model For Portfolio Management with Mortgage-Backed Securities, Decision Sciences Department, The Wharton School, University of Pennsylvania, Philadelphia, PA, to appear in Annals of Operations Research. 


\section{List of Figures}

2.1 Additive approximation versus true cost function $\ldots \ldots \ldots . \ldots 22$

2.2 Decision tree for the special class of "here and now" multi-stage problems 33

3.1 Hypercube multicomputers of dimensions $n \leq 4 \ldots \ldots \ldots \ldots$

3.2 The parallel algorithm $\ldots \ldots \ldots \ldots \ldots \ldots \ldots$

3.3 Efficiency versus sample size $\ldots \ldots \ldots \ldots \ldots \ldots \ldots \ldots$

3.4 Efficiency versus number of processors $\ldots \ldots \ldots \ldots \ldots$

4.1 Decision tree of a general class of "wait and see" multi-stage problems 61

4.2 Path sampling for upper bounds . . . . . . . . . . . . . 83

4.3 Sample points obtained from path sampling . . . . . . . . 84 


\section{List of Tables}

2.1 Model APL1P, test problem data . . . . . . . . . . . 36

2.2 Model APL1P, 20 samples (100 replications of the experiment) . . . . 37

2.3 Model APL1P, 200 samples (100 replications of the experiment) . . . 38

2.4 Model PGP2, 50 samples (100 replications of the experiment) . . . . 40

2.5 Model CEP1, 200 samples (100 replications of the experiment) . . . 41

2.6 Large test problems: computational results for power planning . . . . 42

2.7 Large test problems: computational results for financial planning . . . 43

3.1 Warm start all subs . . . . . . . . . . . . . . . . 54

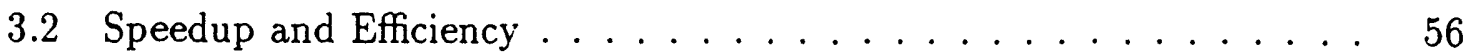

3.3 Optimal Solution . . . . . . . . . . . . . . . . 58 

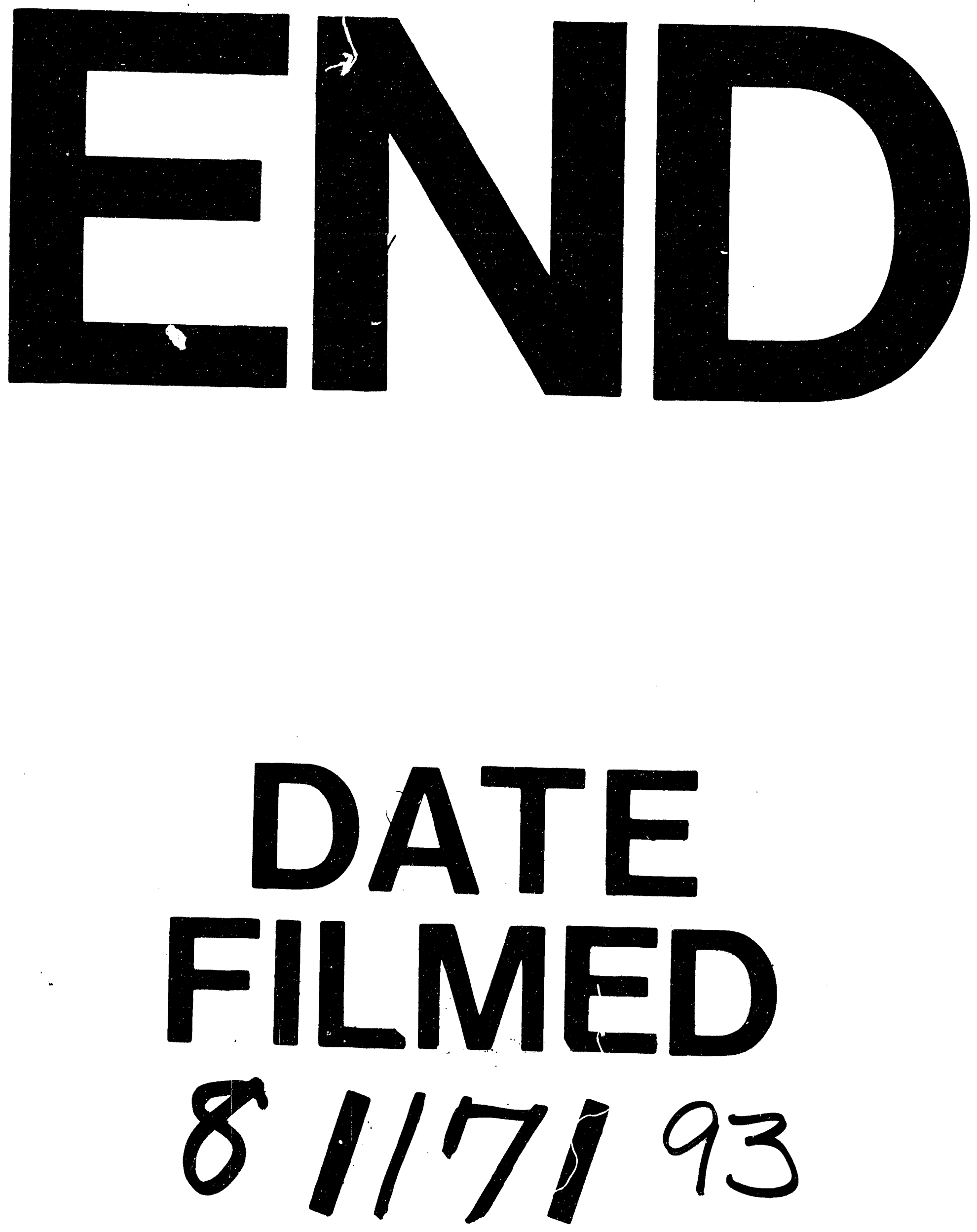
